Benoy Kumar Mukherjee

A Thesis Submitted for the Degree of PhD

at the

University of St Andrews

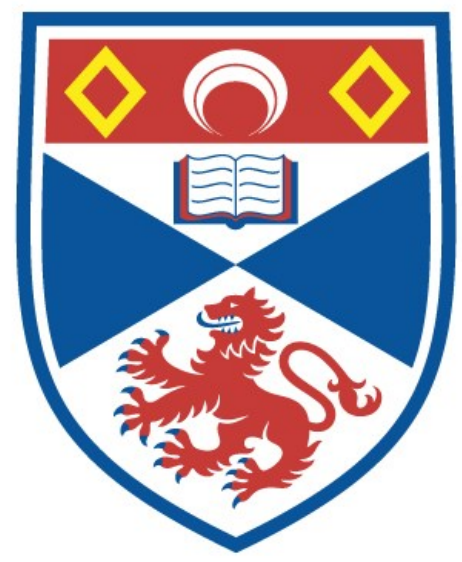

1971

Full metadata for this item is available in

St Andrews Research Repository

at:

http://research-repository.st-andrews.ac.uk/

Please use this identifier to cite or link to this item:

http://hdl.handle.net/10023/14670

This item is protected by original copyright 
DESTRUCTION OF SUPERCONDUCTIVITY BY A CURRENT

\author{
A thesis presented by \\ Benoy Kumar Mukherjee, B.Sc., \\ to the University of St. Andrews \\ in application for the degree of \\ Doctor of Philosophy.
}


All rights reserved

INFORMATION TO ALL USERS

The quality of this reproduction is dependent upon the quality of the copy submitted.

In the unlikely event that the author did not send a complete manuscript and there are missing pages, these will be noted. Also, if material had to be removed, a note will indicate the deletion.

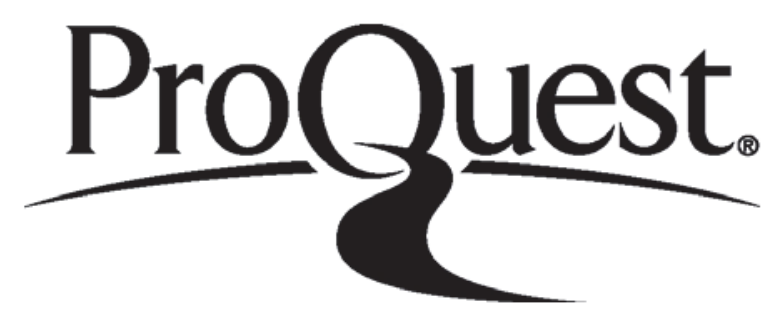

ProQuest 10171262

Published by ProQuest LLC (2017). Copyright of the Dissertation is held by the Author.

All rights reserved.

This work is protected against unauthorized copying under Title 17, United States Code Microform Edition (C) ProQuest LLC.

ProQuest LLC.

789 East Eisenhower Parkway

P.O. Box 1346

Ann Arbor, MI 48106 - 1346 
Th 5882 


\section{'DECLARATION}

I hereby certify that this thesis has been composed by me, and is a record of work done by me, and has not previously been presented for a Higher Degree.

The research was carried out in the School of Physical Sciences in the University of St. Andrews, under the supervision of Professor J: F. Allen, F.R.S.

Benoy Kumar Mukherjee 
CERTIFICATE

I certify that Benoy Kumar Mukherjee, B.Sc.. has spent nine terms at research work in the School of Physical Sciences in the University of St. Andrews under my direction, that he has fulfilled the conditions of the Resolution of the University Court. 1967, No. 1, and that he is qualified to submit the accompanying thesis in application for the Degree of Doctor of Philosophy.

Research Supervisor 


\section{PERSONAL PREFACE}

I first matriculated in St. Salvator's College, University of St. Andrews, in October 1962 and, in 1965, I graduated B. Sc. with first class honours in Physics. In October 1966 I started research in the School of Physical Sciences, University of St. Andrews, under the supervision of Professor J.F. Allen. At the same time I was admitted as a research student under Ordinance General No.12, and in October 1967 I was admitted as a candidate for the degree of $\mathrm{Ph} . \mathrm{D}$ under Ordinance No.88. I continued my research at St. Andrews until November 1969 and during these three years I was employed by the University of St. Andrews as a Demonstrator in Physics. 


\section{ABSTRACT}

We have carried out an experimental investigation of the resistance transition in Indium and Thallium wires when superconductivity is destroyed by a current. Our results, as well as those obtained previously by other workers, do not agree with the theories put forward by London(1937) and Gorter(1957) and the consideration of secondary effects does not satisfactorily account for the discrepancies. We present a new model of the intermediate state in Type-I current-carrying superconductors. In addition to predicting a resistance transition in reasonable agreement with experimental observations, the model gives good agreement with experimental values of the radius of the intermediate state core as obtained by Rinderer(1956). A treatment of secondary effects is also given and together with the basic resistance transition predicted by the model, they provide a better understanding of the destruction of superconductivity in Type-I wires by a current. 
Certificate

Personal Preface

.

Abstract

Table of Contents

Chapter 1. Superconductivity

1.1 Introduction

1.1

1.2 Thermodynamics of Superconductivity. The Two Fluid Model

1. 3 The Electrodynamics of Superconductivity

1.4 Microscopic Theories of Superconductivity

1.5 Type-II Superconductors. The Ginzburg-Landau Equations

Chapter 2. The Current-Induced Intermediate State

2. 1 The Intermediate State

2.2 The London Theory

2. 3 Gorter's Dynamic Model

2. 4 Plan of this Work

Chapter 3. The Experimental Investigation

3. I Introduction

3.2. The Electrical Circuit

3. 3 The Cryogenic Set-up

3.4 The Specimens

3.5 The Experimental Results

3. 6 Discussion

Chapter 4. The Search for a Satisfactory Model

4.1 Discussion of the London Model

4.2 An alternative criterion

4.3 Finding the Structure

4.4 The Relaxational Solution of Laplace's Equation

4.5 Application to the present problem

Chapter 5. The New Model

5.1 The New Model $\left(i=i_{c}\right)$

5.2 The Supercooling involved in the structure

5. 3 The New Model ( $i<i_{c}$ )

5.4 Comparision with Experiment. The Resistance Transition 
5.5 Comparision with Experiment. Radius of the Intermediate State Core

5.12

5.6 Secondary Effects. I. Joule Heating

5.13

5.7 Secondary Effects. II. The Size Effect

Chapter 6. Andreev's Theory

6.1 Andreev's model

6. 1

6.2 Compaxision with experiment

6. 2

6. 3 Theoretical considerations

6. 3

Chapter 7. Summary and Conclusions.

References

8. 1

Acknowledgements

8.3

Appendix I. A Typical Computer Programme

Appendix II. Publications based on this work 


\section{SUPERCONDUCTIVITY}

\subsection{INTRODUCTION:}

Soon after Kamerlingh Onnes first liquified Helium in 1908, he started investigating the resistivity of metals in the newly attainable range of low temperatures. In 1911 he observed (Onnes, 1911) that the resistance of a sample of mercury dropped from about $0.08 \Omega$ to less than $3 \times 10^{-6} \Omega$ at about $4^{\circ} \mathrm{K}$ and that the drop occurred over a temperature interval of less than $0 . I^{\circ} \mathrm{K}$. Onnes recognised that he had discovered a. new state of matter, characterised by 'zero' ohmic resistance, which he called the superconducting state. While it can never be proved by experiment that the resistance of a superconductor is in fact zero, no experiment has been able to detect any resistance in the superconducting state. Recent experiments have shown that the resistivity of lead in the superconducting state is less than $3.6 \times 10^{-2} 3 . \mathrm{cm}$ (Quinn and Ittner, 1962) and that the transition width in lead is less than $3 \times 10^{-4} \mathrm{o}_{\mathrm{K}}$ (Neighbor et al, 1965). Superconductivity is now known to occur in over twenty elements and in hundreds of alloys and compounds of both superconductive and non-superconductive elements.

The temperature below which a metal is superconducting is termed its critical or transition temperature $T_{c}$ and is characteristic of the superconductor. At any temperature below $\mathbf{w}_{c}$, superconductivity can be destroyed by the application of a minimum magnetic field $\mathrm{H}_{c}$, called the thermodynamic critical field, which is a function of temperature. For all superconductors the variation of critical field with temperature is given, to within a few percent, by the relation:

$$
H_{c}(T)=H_{0}\left[1-\left(T / T_{c}\right)^{2}\right]
$$

where $B_{0}$ is the critical field at absolute zero. Superconductivity can also be destroyed by passing a minimum current $i_{c}$, called the critical current, through a specimen. Silsbee's hypothesis (Silsbee, 1916) states that the 
critical current is that which produces a magnetic field equal to the critical field at the surface of the specimen.

The property of perfect conductivity implies that there is no electric field inside a superconductor and it follows from one of Maxwell's equations that

$$
\underline{\dot{B}}=\partial B / \partial t \cdot=-c \operatorname{curl} \underline{E}=0
$$

i. $\theta$. the magnetic induction $B$ in a superconductor is independent of time and will always remain equal to the value of $\underline{B}$ at the instant the specimen becomes superconducting. However Heissner and Ochsenfeld (1933) demonstrated experimentally that the magnetic induction inside a bulk superconductor is always zero:

$$
\underline{B} \equiv 0
$$

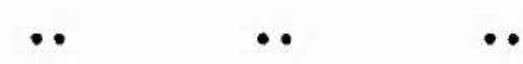

irrespective of the circumstances under which the specinen becomes superconducting. This property, commonly knom as the Meissner effect, implies that a superconductor is a perfect diamagnet in addition to being a perfect conductor.

\subsection{THERMODYNAMICS OF SUPERCONDUCTIVITY. THE TWO FLUID NODEL.}

-It follows from the Heissner effeet that the normal-superconducting transition is reversible so that ordinary thermodynamics can be applied to it. By equating the Gibbs Free Energy in the two phases it is easy to obtain the following expressions (see for example shoenberg, 1952):

$$
\begin{aligned}
& \mathrm{G}_{\mathrm{n}}(0)-\mathrm{G}_{\mathrm{S}}(0)=\mathrm{v} \cdot \mathrm{H}_{0}^{2} / 8 \pi \\
& s_{s}-s_{n}=\mu_{0} V_{c}\left(d H_{c} / d T\right) \\
& c_{s}-c_{n}=T(d S / d T) \\
& =\mu_{0} V_{c}\left(d^{2} H_{c} / d T^{2}\right)+\mu_{o} \operatorname{VT}\left(d H_{c} / d T\right)^{2} \\
& I=T A S=-\mu_{0} \mathrm{VHH}_{\mathrm{c}}\left(\mathrm{aH}_{\mathrm{c}} / \mathrm{dT}\right)
\end{aligned}
$$

where $G_{n}(0), G_{s}(0), c_{n}, c_{s}, S_{n}, s_{s}$ are the free energies in zero magnetic field; specific heats and entropies respectively in the normal and superconductive phases, $I$ is the latent heat of the phase trarisition and $V$ is the volume of the material. 
If we use equation (1.1) for the variation of the critical field with temperature, we get:

$$
C_{s}-C_{n}=6 \mu_{0} V\left(H_{0}^{2} / T_{c}\right)\left[\left(T / T_{c}\right)^{3}-\left(T / 3 T_{c}\right)\right]
$$

In the absence of any definite experimental evidence to the contrary, we may assume that the properties of the lattice are unchanged during the transition so that expression (1.7) really represents the difference in electronic specific heats between the normal and superconducting states. Since it is known that at low temperatures the electronic specific heat of metals is of the same order as the second term of expression (1.7), we may conclude that the electronic specific heat $C_{e s}$ of superconductors is proportional to $T^{3}$. Farly experimental results were thought to confirm this $\mathrm{T}^{3}$ dependence of $\mathrm{C}_{e s}$ as well as the parabolio $T$ dependence of the critical field $\mathrm{H}_{\mathrm{c}^{*}}$

In 1934 Gorter and Casimir formulated a phenomenological two-fluid model of superconductivity based on the assumption that in the superconducting state a fraction $x$ of the conduction electrons are condensed into an ordered state (such electrons may be termed 'superelectrons') and do not contribute to the entropy of the system while the remaining fraction ( $1-x$ ) remain 'normal' and have the same properties as conduction electrons in the normal state. If $B_{\mathbb{S}}(\mathrm{T})$ and $g_{n}(T)$ represent the free energies per unit volume of the superconducting and normal electrons, the total Gibbs free energy per unit volume of a superconductor may be written:

$$
G_{S}(T)=F(x) \cdot g_{S}(T)+F \cdot(I-x) \cdot g_{n}(T)
$$

from which expressions may be calculated for the entropy and the specific heat of the zupsrconducting state. In order to obtain the $\mathrm{T}^{3}$ dependence of the specific heat, Gorter and Casimir were led to assume $F(x)=x$ and $F^{\prime}(1-x)=(1-x)^{\frac{1}{2}}$ which then led to the following results:

$$
\begin{aligned}
& c_{e s}=3 \gamma T_{c}\left(T / T_{c}\right)^{3} \\
& S_{s}=\gamma T_{c}\left(T / T_{c}\right)^{3} \\
& x(T)=1-\left(T / T_{c}\right)^{4}
\end{aligned}
$$


However later and more careful measurements showed that the $\mathrm{T}^{3}$ dependence of $\mathrm{C}_{e s}$ and the $\mathrm{T}^{2}$ dependence of the critical field were not strictly correct. For example, the specific heat can be more accurately represented by an exponential function. Thus the Gorter-Casimir model does not provide a quantitative understanding of superconductivity; nevertheless the two-fluid concept does help qualitatively in understanding later microscopic theories.

\section{3 THE ELECTRODYNAMICS OF SUPERCONDUCTIVITY:}

In 1935, F. London and H.London showed that the electrodynamics of a superconductor could be adequately described by the addition of two equations to the usual Maxwell's equations of electromagnetism. These 'London equations' are:

$$
\begin{aligned}
& \underline{\dot{J}}=\left(n_{s} e^{2} / m\right) \underline{E} \\
& \underline{B}=-\left(m / n_{s} e^{2}\right) \operatorname{curl} \underline{J}
\end{aligned}
$$

where $\mathrm{J}$ is the current density in the superconductor, $\underline{B}$ and $\underline{E}$ are respectively the magnetic and electric fields in the material, $n_{s}$ is the number of superconducting electrons per-unit-volume-and-ma and are their mass and charge respectively. Equation (1.12) describes the perfect conductivity of a superconductor while equation. (1.13) describes the property of perfect diamagnetism.

The London equations together with Maxwell's equations may be used to show that the magnetic field inside the superconductor satisfies the equation:

$$
\nabla^{2} \underline{B}=\left(1 / \lambda_{L}^{2}\right) \underline{B} \text {. with } \lambda_{L}^{2}=\left(m / \mu_{0} n_{S} e^{2}\right) \quad \cdots
$$

This shows that the magnetic induction decreases exponentially inside a superconductor falling to $1 / e$ of its value at the surface at a distance of $\lambda_{L}$, which is called the London penetration depth. Thus the London equations lead to the Meissner effect mentioned previously; the existence of the penetration depth has also been confirmed although experimental values are not in good quantitative agreement with $\lambda_{L}$. 
In equation (1.14) defining $\lambda_{L}$, the density $n_{S}$ of superconducting electrons: is the only temperature dependent factor and by using (1.11) we obtain

$$
\lambda_{L}(T)=\lambda_{L}(0)\left[I-\left(T / T_{C}\right)^{4}\right]^{-\frac{1}{2}}
$$

where $\lambda_{\mathrm{L}}(0)$ is the penetration depth at absolute zero. Although experimental results obtained by Daunt et al (1948) could be represented reasonably well by (1.15), more precise measurements by Schawlow (1958) show deviations at low temperatures and give better agreement with the BCS theory to be described later.

In the course of an extensive investigation of the high frequency surface impedance of superconductors Pippard noted a number of experimental facts which could not be understood on the basis of the London equations: (a) the penetration depth is anisotropic in single crystals, (b) the presence of a small amount of impurity changes the penetration depth considerably, (c) $\lambda$ is almost independent of any externally applied magnetic field near $T_{c}$ which implies a large change in the entropy density within the penetration depth. These led Pippard (1953) to conclude that superconductivity involved a rather long range interaction within the electron assembly so that a perturbation at one point would be felt over a distance $\xi$, called the range of coherence or coherence length, and similarly the response of any point to a spatially extended perturbation could only be obtained by integrating over a region surrounding the point Pippard modified the London equations to take account of the range of coherence and proposed that the electromagnetic behaviour of a superconductor would be governed by the relation:

$$
J(R)=\left(3 n e^{2} / 4 \pi \xi_{0} m\right) \int \underline{R}(\underline{R} \cdot \underline{A}) e^{-R / \xi} \mathrm{d} \tau / R^{4}
$$

where $\underline{R}$ is the position vector, $\underline{A}$ is the electromagnetic vector potential, $\xi_{0}$ is the coherence length of the pure superconductor and $\xi(I)$ is a parameter depending on the mean free path. The penetration depth is now a function of the mean free path:

$$
\lambda=\lambda_{L}\left(\xi_{0} / \xi(1)\right)^{\frac{1}{2}}
$$


where $\lambda_{I}$ is the London value of the penetration depth. Pippard found that. his experimental findings on the mean free path dependence of the penetration depth could be understood by assuming a relationship of the form:

$$
1 / \xi(1)=1 / \xi_{0}+1 / \alpha 1
$$

where $a$ is a constant of order unity.

The existence of a penetration depth and a coherence length implies that the boundary between normal and superconducting parts of the same specimen will have a free energy per unit volume different from that on either side. In other words the boundary may be said to have a surface energy $\alpha$ per unit area. The surface energy is given approximately by:

$$
\alpha=(\xi-\lambda) \cdot\left(\mathrm{H}_{\mathrm{c}}^{2} / 8 \pi\right)=\Delta \mathrm{H}_{\mathrm{c}}^{2} / 8 \pi
$$

where $\Delta$ is called the surface energy parameter and has the dimension of length.

Well before Pippard introduced the concept of coherence length, H. London had recognised that the Meissner effect required the existence of a positive surface energy as otherwise a specimen in the presence of a magnetic field would break up into normal and superconducting layers of thickness $d_{n}$ and $d_{s}$, with $d_{n} \ll d_{s}<\lambda$ and still have a lower energy than the Meissner state.

\subsection{MICROSCOPIC THEORIES OF SUPERCONDUCTIVITY:}

Thus far we have considered superconductivity in purely macroscopic terms; for example in the two-fluid model we have considered superelectrons without discussing how this state arises. Unfortunately the complete microscopic theory of superconductivity involves advanced quantum mechanics and complicated mathematics. Besides, the work to be described in later chapters can largely be understood on the basis of the macroscopic theories already outlined. We shall therefore only give a brief qualitative discussion of the basic physical ideas involved in the macroscopic theory of superconductivity.

Since superconductivity involves the general properties of electrons in solids, its explanation had to await the development of quantum mechanics and 
its appication to the normal state. The absence of any significant change in the lattice properties of a specimen during the phase transition and the sharpness of the changes in thermodynamic and other properties suggested that superconductivity was caused by an interaction between electrons. Numerous attempts were made to use the Coulomb repulsive interaction between electrons to account for superconductivity but they were unsuccessful. Frohlich (1950) first suggested the interaction which is now believed to be responsible for superconductivity. The Frohlich interaction consists of the exchange: of momentum between two electrons with a phonon acting as intermediary. Thus an electron with momentum $\mathrm{k}_{1}$ can emit a phonon of momentum $\mathrm{q}$ which will be absorbed by another electron with initial momentum $k_{2}$. The net effect is to change the electrons' momenta from $k_{1}$, $k_{2}$ to $k_{1}-q, k_{2}+q$ The Frohlich interaction depends on the energies $\epsilon_{1}$ and $\epsilon_{2}$ of the participating electrons and it can be show that the interaction is attractive if $\left|\epsilon_{1}-\epsilon_{2}\right|$ is sufficiently small whereas it is repulsive if $\left|\epsilon_{1}-\epsilon_{2}\right|$ is sufficiently large.

If the Frohlich interaction is indeed the decisive interaction producing superconductivity, two important consequences follow. Firstly, since the interaction involves phonons, superconductivity must depend on the properties of the ionic lattice and Frohlich predicted that the transition temperature should depend on the mass of the ion cores. Experimental investigation (Maxwel1, 1950; Reynolds et al, 1950) of different isotopes of a number of superconducting metals confirmed Frohlich's prediction and showed that in the case of almost all non-transition metals one could write:

$$
\mathrm{T}_{\mathrm{c}} \mathrm{N}^{\frac{1}{2}}=\text { constant }
$$

where $M$ is the isotopic mass.

Secondly, the Frohlich interaction will only be significant if the electronphonon interaction is relatively strong which implies poor electrical conductivity in the normal state. Thus the poor metallic conductors are more likely to exhibit superconductivity than the better metallic conductors and this is 
confirmed by experiment.

In 1956 I. N. Cooper showed that even a weak electron-electron interaction (such as the Frohlich interaction) together with the Pauli exclusion principle could lead to the formation of stable, bound pairs of electrons. Consider, for example, two electrons with energy and momentum $\epsilon_{1}, k_{1}$ and $\epsilon_{2}$, $k_{2}$ respectively. As a result of an attractive interaction they could form a bound state with energy $\epsilon_{1}+\epsilon_{2}-2 \Delta$ where $2 \Delta$ is the binding energy due to the interaction. Presumably the electrons could break free of each other with new values of energy $\epsilon_{1}^{\prime}=\epsilon_{1}-\Delta$ and $\epsilon_{2}^{\prime}=\epsilon_{2}-\Delta$. However the new momentum values $k_{1}^{\prime}$ and $k_{2}^{\prime}$ corresponding to $\epsilon_{1}^{\prime}$ and $\epsilon_{2}^{\prime}$ may already be possessed by other electrons and would therefore be forbidden to the original electrons by the Pauli exclusion principle thus forcing them to remain in the bound state. It turns out that the Froblich attractive interaction is strongest when $\epsilon_{1}=\epsilon_{2}$ i. e. $\mathrm{k}_{1}=\mathrm{k}_{2}$ and when the electrons have opposite spins. Such a pair of bound electrons is known as a Cooper pair.

The pairing effect led Bardeen, Cooper and Schrieffer (hereinafter referred to as BCS) to assume that a superconductor at absolute zero had all its electrons paired off in Cooper pairs. They (and also Bogoliubov et al, 1958) showed that such a system of Cooper pairs had a lower energy than the free electron model of the normal state and would therefore be the thermodynamically favoured system. Their detailed mathematical treatment further demonstrated that a system of Cooper pairs would indeed exhibit the Meissner effect and the property of resistanceless current flow.

At non-zero temperatures, the thermal energy present causes the break up of some of the Cooper pairs into seperate unpaired electrons which are often called quasi-particles. As the temperature is increased, the number of quasiparticles will increase while the number of Cooper pairs will decrease. We thus get back to the qualitative picture of the two-fluid model described 
The break up of a Cooper pair requires a minimum energy equal to the binding energy of the pair, say $2 \Delta$. In other words a minimum energy of $\Delta$ per electron is required to create two quasi-particles. There is thus an energy gap $\Delta$ between a paired and an unpaired electron and it follows that, at low temperatures $\mathrm{T}$, the number of unpaired electrons will be proportional to $\exp (-\Delta / \mathrm{kT})$ where $\mathrm{k}$ is Boltzmann's constant. Since the unpaired electrons are responsible for the electronic specific heat of a superconductor, the latter should also be expected to have a similar temperature dependence. Careful experiments have shown that $C_{e s}$ is indeed proportional to $\exp (-\Delta / \mathrm{kT})$ rather than to $\mathrm{T}^{3}$ (see for example Lynton, 1969) Further confirmation of the existence of the energy gap comes from experiments on thermal conductivity, ultrasonic attenuation, absorption of electromagnetic energy, tunnelling of electrons from a superconductor to another superconductor or ${ }_{n}$ normal metal, the results of all of which can be understood on the basis of the BCS theory. The energy gap $\Delta$ drops very slowly as the temperature $\mathrm{T}$ increases from $0^{\circ} \mathrm{K}$ until $\mathrm{T} \sim \mathrm{T}_{\mathrm{c}} / 2$ when it begins to fall more rapidly, approaching zero at $\mathrm{T}_{\mathrm{c}}$ with a vertical tangent. Near $T_{c}, \Delta$ may be expressed as:

$$
\Delta(\mathrm{T}) \sim 3.2 \mathrm{kT} \mathrm{T}_{\mathrm{c}}\left[1-\left(\mathrm{T} / \mathrm{T}_{\mathrm{c}}\right)\right]^{\frac{2}{2}}
$$

Cooper (1956) has pointed out that the size of the wavefunction of a Cooper pair (in other words the mean distance between the two paired electrons) is of the order of $10^{-4} \mathrm{~cm}$. Thus the existence of Cooper pairs provides an explanation of the concept of coherence length introduced earlier by Pippard. Indeed equation (1.16) proposed by Pippard can be derived from the BCS theory.

In general the BCS theory has been remarkably successful in explaining the properties of superconductors. However, in the case of the transition metals, a number of discrepancies exist and several more or less successful attempts have been made to extend or modify the theory to explain each discrepancy as it has arisen. Natthias (1969) has suggested that perhaps a mechanism other than the BCS one is responsible for superconductivity in these 
metals.

1.5 TYPE-II SUPERCONDUCTORS. THE GINZBURG-LANDAU EQUATIONS.

In some superconducting metals and most superconducting alloys the coherence length happens to be smaller than the penetration depth so that the interphase surface energy in these substances is negative and the formation of such surfaces becomes energetically favourable. In the presence of an : applied magnetic field these substances would not exhibit the Meissner effect but would rather split into a fine mixture of superconducting and normal regions in such a way as to maximise the interphase surface area relative to the volume of the normal regions. Such a state is called the mixed state and superconductors with a negative surface energy are designated Type-II superconductors as distinct from $1 y p e-I$ superconductors which have a positive surface energy and exhibit the Meissner effect.

In 1950 Ginzburg and Landau (G-L) proposed a phenomenological theory which is particularly useful in treating superconductors in the presence of a magnetic field as for example in the mixed state. G-I assumed that the behaviour of the superconducting electrons may be described by an effective wave function such that $|\Psi|^{2}=n_{S}$, the density of the superconducting electrons. At temperatures near $T_{c}$ the free energy of the superconducting state differs from that of the normal state by an amount which can be expressed in the form of a power series in $|\Psi|^{2}$ :

$$
G_{s}(0)=G_{n}(0)+a|\Psi|^{2}+(\beta / 2)|\Psi|^{4}+\ldots \ldots
$$

Minimising $G_{S}(0)$ with respect to $|\Psi|^{2}$ gives the zero field value

$$
\left|\Psi_{0}\right|^{2}=-a / \beta
$$

and

$$
G_{s}(0)=G_{n}(0)-\alpha^{2} / 2 \beta \quad \ldots \quad \ldots
$$

G-I assumed:

$$
\begin{aligned}
& \boldsymbol{a}(\mathrm{T})=\left(\mathrm{T}_{\mathrm{c}}-\mathrm{T}\right) \cdot(\mathrm{d} \alpha / d \mathrm{~T})_{\mathrm{T}=\mathrm{T}_{\mathrm{C}}} \\
& \boldsymbol{\beta}(\mathrm{T})=\beta\left(\mathrm{T}_{\mathrm{c}}\right)=\beta_{\mathrm{c}}
\end{aligned}
$$

so that

$$
H_{c}^{2}=8 \pi\left[G_{n}(0)-G_{S}(0)\right]=\left(4 \pi / \beta_{c}\right) \cdot\left(T_{c}-T\right)^{2}(d u / \alpha T)^{2} T_{=}=T_{c}
$$


which agrees with experiment, thus justifying the assumptions.

In the presence of a magnetic field $\mathrm{H}_{e}$ the wave function $\Psi$ varies spatially. and the free energy of the superconductor is increased not only by the volume term $\mathrm{H}_{e}{ }^{2} / 8 \pi$ but also by a term depending on the gradient of $\Psi$. G-L write

$$
G_{s}\left(H_{\theta}\right)=G_{s}(0)+H_{e}^{2} / 8 \pi+(1 / 2 m)\left[-i n \underline{\nabla}-\theta^{*} / c \cdot \underline{A} \Psi\right]
$$

where $\mathrm{m}$ is the electronic mass, $e^{*}$ is an effective charge and $\mathrm{A}$ is the vector potential of the applied field.

The total free energy is now the volume integral of equation (1.26) and minimising this with respect to $\Psi$ and $\underline{A}$ yield the two Ginzburg-Landau equations:

$$
\begin{gathered}
(1 / 2 \mathrm{~m})\left[-i \hbar \underline{\nabla}-e^{*} / \mathrm{c}\right] \Psi+\partial G_{\mathrm{S}}(0) / \partial \Psi=0 \\
\nabla^{2} \underline{\Psi}=(4 \pi / c) \underline{J}_{S}=\left(2 \pi i \hbar e^{*} / \mathrm{mc}\right)\left(\underline{\Psi} \nabla \bar{\Psi}-\Psi \underline{\Psi} \Psi^{*}\right)+\left(4 \pi e^{*} / \mathrm{mc}^{2}\right)|\bar{\Psi}|^{2} \underline{A}
\end{gathered}
$$

The application of the above equations to a planar boundary leads to a penetration depth in zero magnetic field (weak field limit) of the form

$$
\lambda_{0}=\left(m c^{2} / 4 \pi e^{2} \Psi_{0}^{2}\right)^{\frac{1}{2}}
$$

G-I showed that the interphase surface energy is closely related to a dimensionless quantity

$$
\kappa=\left|\left(2 \theta *^{2} / h^{2} c^{2}\right) H_{c}^{2} \lambda_{0}{ }^{4}\right|^{\frac{1}{2}}
$$

which is characteristic of a superconductor and is commonly known as the Ginzburg-Landau parameter. For $\kappa<<1$, the surface energy parameter is closely. approximated by

$$
\Delta=1.89\left(\lambda_{0} / \kappa\right)
$$

and it can be shown that the surface energy of superconductor is positive or negative depending on whether $\kappa<1 / \sqrt{2}$ or $\kappa>1 / \sqrt{2}$ respectively; thus the value of $1 / \sqrt{2}$ for $k$ defines the boundary between Type-I and Type-II superconductors.

The applicability of the G-I theory as outlined above is restricted to temperatures close to $\boldsymbol{T}_{\mathrm{c}}$, where the order parameter is small and its spatial 
variation is slow. However Gorkov (1959) has show that the G-I equations can be derived from the BCS theory and the theory has since been extended to cover all temperatures. (see e. g. Eilenberger, 1966, and De Gennes, 1966). Nore recently attempts have been made to extend the use of the G-I theory to cases where the order parameter $\Psi$ varies with time (Iucas and Stephen, 1967)

The Ginzburg-Landau equations have been extensively used by Abrikosov (1957), De Gennes (1966) and other workers to successfully describe the properties of Type-II superconductors. However, as the present work is almost solely concerned with Type-I superconductors, the properties of Type-II superconductors will not be reviewed here. 


\section{THE CURRENT-INDUCED INTERMEDIATE STATE.}

\section{I THE INTERMEDIATE STATE:}

A sample of Type-I superconductor is said to be in the intermediate state when it contains coexistent superconducting and normal regions.

There are two major experimental situations which pxoduce an intermediate state. The first is the application of an external magnetic field $\mathrm{H}_{e}$ to a specimen of non-zero demagnetising factor $D$. The diamagnetism of the superconductor distorts the applied field and produces a non-uniform surface field. If the external field is gradually increased, the sample enters the intermediate state at $\mathrm{H}_{e}=\mathrm{H}_{c}(I-D)$ and it becomes fully normal only when $\mathrm{H}_{e}=\mathrm{H}_{c^{\circ}}$

The second major experimental situation which produces an intermediate state is the passage of current through a superconducting wire (cylinder). It is clear that when the applied current reaches the critical value $i_{c}$, the whole wire cannot pass into the normal state. If it did, the current density in the wire would be uniform and at a distance $r$ from the axis of the cylinder the field would be

$$
H(r)=(r / a) \cdot H(a)
$$

where $a$ is the radius of the wire. Thus when the current has the critical i.e.

value, ${ }_{\wedge} \mathrm{H}(\mathrm{a})=\mathrm{H}_{\mathrm{c}}$ by Silsbee's hypothesis, the field near the axis would be well below critical which is incompatible with the assumption that the whole wire had become normal. Thus the wire cannot be fully normal, nor can it be wholly superconducting since the surface field has reached the critical value. The intermediate state produced in this way may be termed the current-induced intermediate state, and the rest of this work is concerned with the study of this state.

\subsection{THE LONDON THEORY:}

We have shown that when a critical current is passed through a superconducting wire the whole wire cannot go into the normal state. A possible alternative would be for the wire to have a superconducting core of radius $r_{0}$ 
along the axis with a normal sheath outside it. Then the core would carry the entire current and the field at the core boundary would be :

$$
H\left(r_{0}\right)=i_{c} / 2 \pi r_{0}>i_{c} / 2 \pi a=H(a)=H_{c}
$$

so that the boundary would not be in equilibrium. Thus the simple model of a superconducting core surrounded by a normal sheath is not self-consistent and we are led to assume that the core itself must be in the intermediate state. At equilibrium superconducting-normal interfaces must be electric equipotentials so that if the intermediate state structure is laminar, the laminae must be oriented perpendicularly to the direction of current flow.

These considerations led F. London (1935) to suggest that for $i_{c}<i$ the wire would have an intermediate state core of radius $r_{c}<$ a surrounded by a cylindrical sheath in the normal state as shown in Fig.2.1. The normal sheath corresponds to the region where the magnetic field is greater than $H_{c^{*}}$ London assumed that everywhere inside the core the field is $\mathrm{H}_{C}$, so that the current flowing within any radius $r<r_{c}$ should be

$$
i(r)=2 \pi \mathrm{rH}_{c}
$$

and, in particular, the total current carried by the intermediate state core is

$$
i_{\text {core }}=i\left(r_{c}\right)=2 \pi r_{c} H_{c} \quad \cdots
$$

At the surface of the core the normal regions merge continuously into the

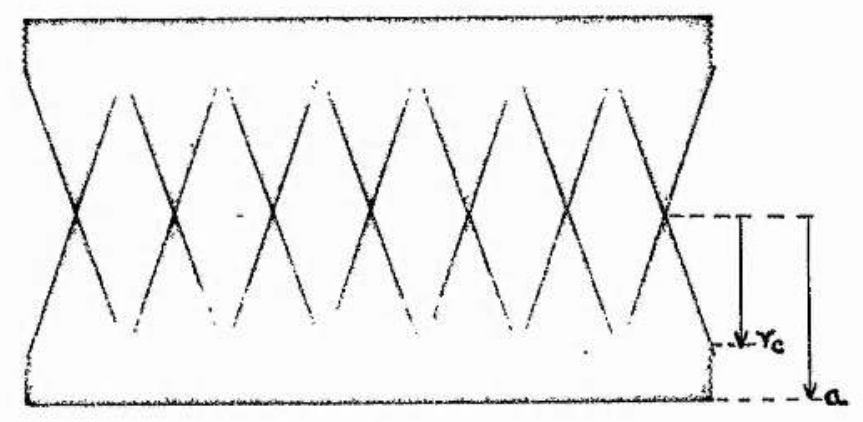

Fig.2.1 Structure proposed by London for the intermediate state in a cylindrical wire in which resistance has been partially restored by a current. The dark areas represent normal regions. 
sheath so that there cannot be any discontinuity in the current density at the core boundary $\left(r=r_{c}\right)_{0}$ Within the core the current density is given by

$$
J(r)=(1 / 2 \pi r) \cdot(d i / d r)=H_{c} / r
$$

In the normal sheath itself the current density must be uniform so that we can write

$$
J_{\text {sheath }}=J\left(r_{c}\right)=H_{c} / r_{c} \quad \cdots
$$

Hence the total sheath current is:

$$
i_{\text {sheath }}=\pi\left(a^{2}-r_{c}^{2}\right) J_{\text {sheath }}=\pi H_{c}\left(a^{2}-r_{c}^{2}\right) / r_{c}
$$

Since the sheath is fully normal, its resistance per unit length is

$$
R_{\text {sheath }}=R_{n} a^{2} /\left(a^{2}-r_{c}^{2}\right)
$$

where $R_{n}$ is the normal resistance per unit length of wire. The electric field per unit length of wire will be

$$
\mathrm{E}=\mathrm{Ri}=\mathrm{R}\left(i_{\text {sheath }}+i_{\text {core }}\right)
$$

or, considering the sheath only,

$$
\mathrm{E}=\mathrm{R}_{\text {sheath }} i_{\text {sheath }}=\mathrm{R}_{\mathrm{n}} \pi \mathrm{a}^{2} \mathrm{H}_{\mathrm{c}} / \mathrm{r}_{\mathrm{c}}
$$

It follows that

$$
R=E / i=\left[\frac{1}{2} R_{n} 1+\left\{1-\left(2 \pi a H_{c} / i\right)^{2}\right\}\right]^{\frac{1}{2}}=\frac{1}{2} R_{n}\left(1+\sqrt{1-\left(i_{c} / i\right)^{2}}\right)
$$

Thus the Iondon model predicts that the resistance of the wire should rise discontinuously to half its normal value as soon as the current reaches its critical value and then continue to rise gradually as the current is further increased, reaching its full value asymptotically. The return of resistance has been experimentally investigated by a number of workers (see for example, Scott 1958, Rinderer 1956, Meissner and Zdanis 1958, Freud et al 1968). Their results agree qualitatively with the London predictions but quantitative agreement is poor. The discontinuous jump $\rho$ in the value of $R / R_{n}$ when the current is critical is found to vary between 0.7 and 0.9 depending on such factors as wire diameter and purity as compared with the value of 0.5 given by London's theory. For $i_{c}<i$ the experimental values of resistance are generally higher than those given by expression (2.9) as 


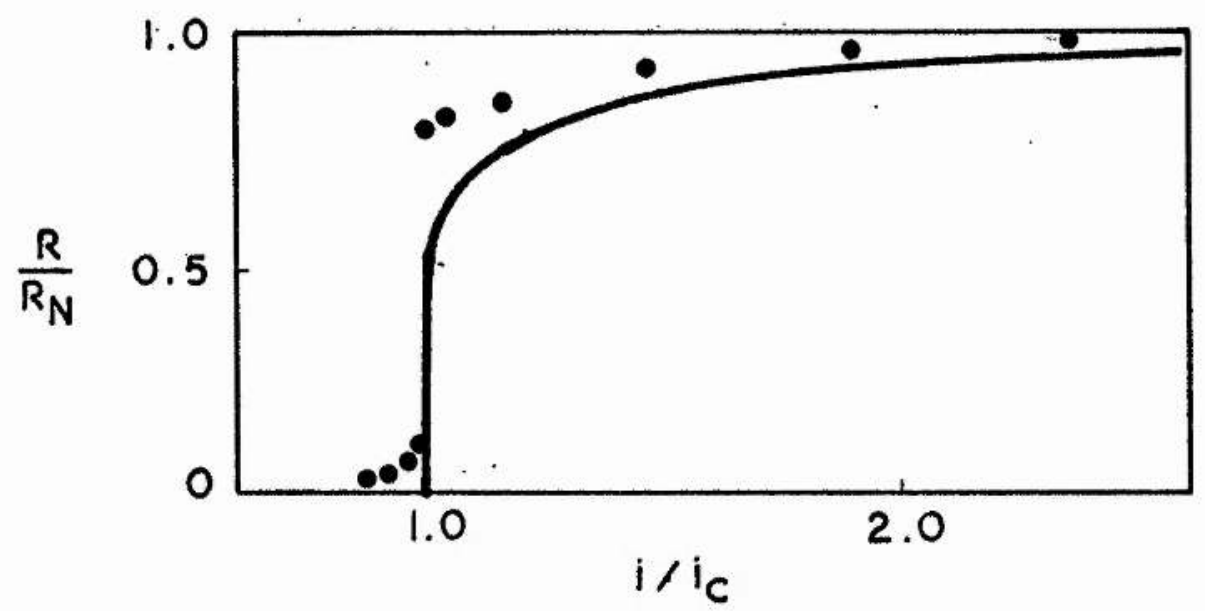

Fig.2.2 The resistance transition predicted by London (solid line) compared with experimental values obtained by Scott (1948) for $0.286 \mathrm{~mm}$. diameter Indium wire.

shown in Fig. 2.2 where Scott's results for a $0.286 \mathrm{~mm}$. diameter Indium wire are compared with the curve of (2.9).

Several attempts have been made to explain the discrepancy between London's theory and experimental results. Scott (1948) found that the value of the resistance jump $\rho$ varies approximately inversely with the wire diameter and suggested that London's expression (2.9) might hold for thick wires. However later work on thick wires by Freud et al (1968) has shown that this was not the case. Kuper (1952) attributed the discrepancy to additional resistance due to the scattering of conduction electrons at the interphase boundaries. An accurate calculation of this effect is difficult because London's model does not specify the periodicity of the structure. Kuper's approximate calculations showed that boundary scattering would indeed increase the value of resistance, in particular that of $\rho$, but consistent agreement with experimental values was still not obtained.

Troinar (1960) investigated the variation of $\rho$ with temperature for tin samples and made some of his measurements at temperatures below the Helium $\lambda$ point. His results indicate that the value of $\rho$ increases as the critical current becomes larger. Further, for impure specimens with high 
residual resistivity Troinar observed a discontinuous fall in the value of $\rho$ as the temperature is lowered below the $\lambda$ point, but he did not find a similar effect for pure 'thick' samples of low residual resistivity. These findings suggest that the value of $\rho$ depends on and increases with joule heating in the specimen. Berkovich and Lapir (1963) have given a theoretical treatment in which they have modified London's treatment to take account of joule heating. They obtain the following modified formula for the resistance of the wire:

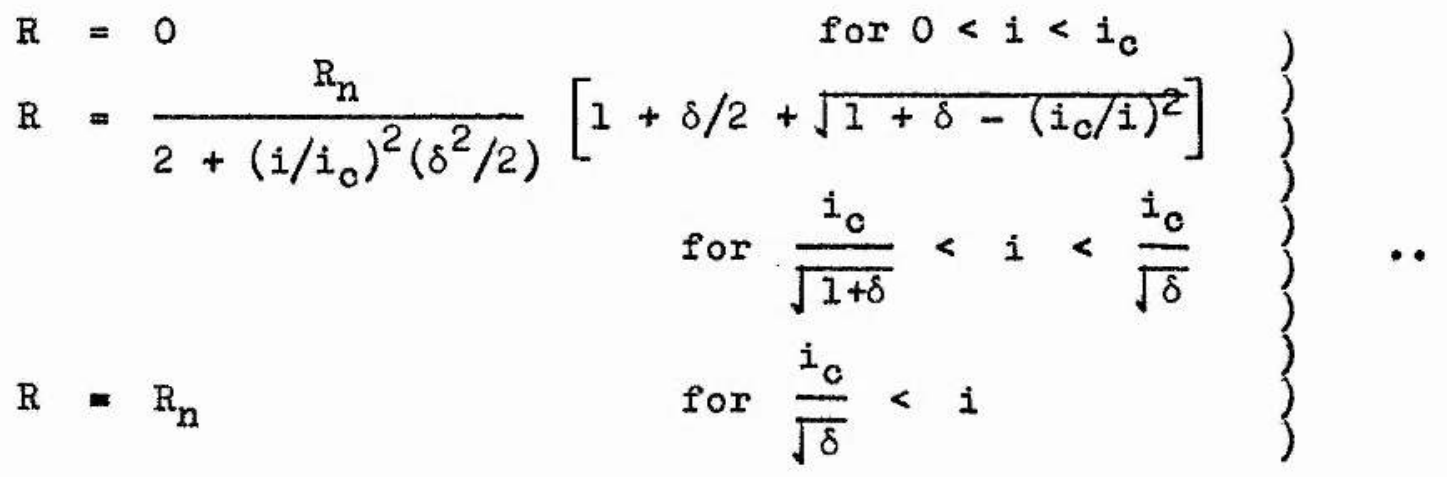

where $\delta=\mathrm{H}_{\mathrm{c}} \mid \frac{\mathrm{dH}}{\mathrm{dT}} \mathrm{g}\left(\rho_{\mathrm{O}} / 8 \pi^{2} \mathrm{ha}\right), \rho_{Q}$ is the resistivity of the normal phase and $h$ is the coefficient for heat transfer across the sample - liquid Helium boundary. Berkovich and Lapir find that in the case of samples of relatively high residual resistance the values of $h$ required to fit expression (2.10) to the experimental curves of Troinar lie within the range of values obtained from direct measurements of $h$. However (2.10) does not agree well with Troinar's results for samples of high purity. Besides, Troinar's observations show that even at temperatures close to $T_{c}$, where joule heating effects should be small, the range of values obtained for $\rho$ does not agree with the London value of 0.5 . Thus joule heating alone cannot account for the discrepancy between's London's expression (2.9) and experimental results.

\subsection{GORTER'S DYNAMIC MODEL:}

Gorter (1957) suggested that the boundaries between normal and superconducting regions might tend to orient themselves parallel instead of perpendicular to the direction of current flow. In the model proposed by him the intermediate state consists of cylindrical shells of alternately 


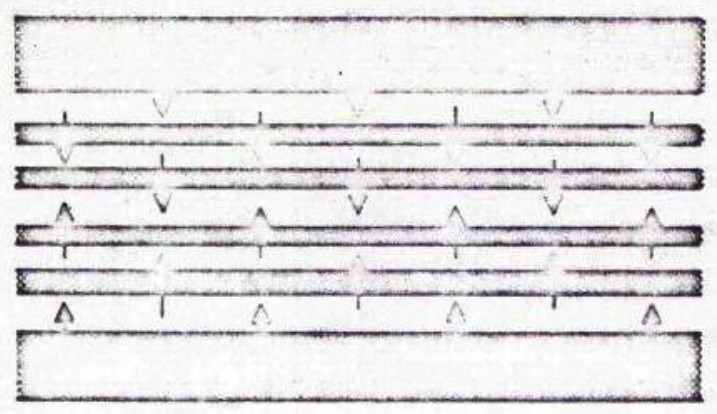

Fig. 2.3 Gorter's dynamic model for the intermediate state structure in a current carrying wire.

normal and superconducting regions as shown in Fig.2.3. Gorter showed that the current through the wire would cause a continuous inward motion of the shells resulting in eddy current losses. Gorter and Potters (1958) calculated the effective resistance due to the eddy current losses and obtained an expression identical to the London expression (2.9). They also showed that if supercooling occurs this leads to an increase in resistance over that given by (2.9) by only a few percent when the current is slightly greater than $i_{c}$; this increase can only account for a fraction of the discrepancy between experimental results and expression (2.9).

In Gorter's model the surface sheath of the wire would alternate between the normal and superconducting states and it follows that the magnetic field produced by the current on the surface of the wire should have a variable component. Shalnikov (1957) attempted to measure this variable component of the field but failed to observe any periodic or non-periodic signals. In another series of experiments Shalnikov used magnetic powder to display the structure of the intermediate state in a wire and found that the superconducting and normal regions oriented themselves perpendicularly to the general direction of current flow.

\section{(1968)}

In a recent theoretical study Kuper and Tait have shown that under ideal conditions Gorter's model is self-consistent only for those Type-I superconductors which have their Ginzburg-Landau parameter in the very limited range 
$0.64<\kappa<0.707$. Thus Gorter's model has not proved satisfactory from either a theoretical or an experimental point of view.

\subsection{PLAN OF THIS WORK:}

We have seen that neither of the models proposed by Londion and Gorter gives quantitative agreement with experimental values on the return of resistance and that Gorter's model also appears to be unsatisfactory from a theoretical point of view.

At the start of this work it was decided to check the experimental situation by some careful measurements of the return of resistance in Indium wires - these measurements are described in the next chapter. A few experiments were sufficient to confirm the general trend of previous results. The fact that various workers under different experimental conditions had all obtained results which were similar in nature, in qualitative agreement with London's model but with a considerable quantitative discrepancy, suggested to us that this discrepancy was perhaps not entirely due to secondary effects. In Chapter 4 a closer look is taken at the London model, it is shown that the model is theoretically unsatisfactory in some ways and the numerical methods used to obtain a more self-consistent model are described. The new model itself is presented in Chapter 5 and it is shown that the return of resistance as predicted by this model agrees better with experimental results than is the case with the models presented earlier in this chapter. The various secondary effects mentioned in connection with London's model may also be applied to the present model and taken together they provide a reasonable understanding of experimental results.

During the course of this work another model vas proposed by Andreev(1968). This is briefly described in Chapter 6 where it is shown that Andreev's model does not agree well with experiment and that it is perhaps not quite satisfactory from a theoretical point of view either. Finally, Chapter 7 concludes the thesis with a review of the work. 


\section{THE EXPERIMENTAL INVESTIGATION}

\subsection{INTRODUCTION:}

As mentioned in the previous chapter, the resistance transition in Type-I wires had been experimentally investigated by a number of workers prior to this work. However in almost all these cases curves showing the return of resistance as a function of current had been obtained by joining discreet experimental (i,R) points. This did not make for the most accurate determination of the resistance jump $\rho$ at the critical current. A prime object of our experimental investigation was to obtain a continuous plot so that the value of $\rho$ could be more accurately determined.

Basically the experiment consisted of passing a very slowly but steadily increasing current through a superconducting wire which was kept at a constant temperature $\mathrm{T}$ just below the critical temperature $\mathrm{T}_{\mathrm{c}}$ and of continuously monitoring the voltage across the wire. The current through the specimen and the voltage across it were plotted on an $X-Y$ recorder which thus plotted a V-I curve for the specimen at that particular temperature. From this it was easy to compute a resistance-current curve for the specimen. Thus for any given specimen a range of $\mathrm{R}-\mathrm{I}$ curves could be obtained at aifferent temperatures close to but below $\mathbf{T}_{\mathrm{c}}$.

\subsection{THE ELECTRICAL CIRCUIT:}

Fig. 3.1 shows a block diagram of the electrical circuit used.

$P$ is a remotely programmable constant current power supply. Two HewlettPackard models - 6824A and 6284A - were used allowing coverage of 0 - 1.2 Amps. and $0-4.0$ Amps. respectively. These models were chosen for good load regulation on constant current operation, for their high stability and low noise characteristics. The current output was controlled by remote resistance programming at approximately 500 ohms per ampere. A ten turn (500 ohm or $2000 \mathrm{ohm}$ ) Beckman potentiometer $\therefore$ R fitted with a digital counter was used as the controlling resistance and a uniform change (increase/decrease) in the current 


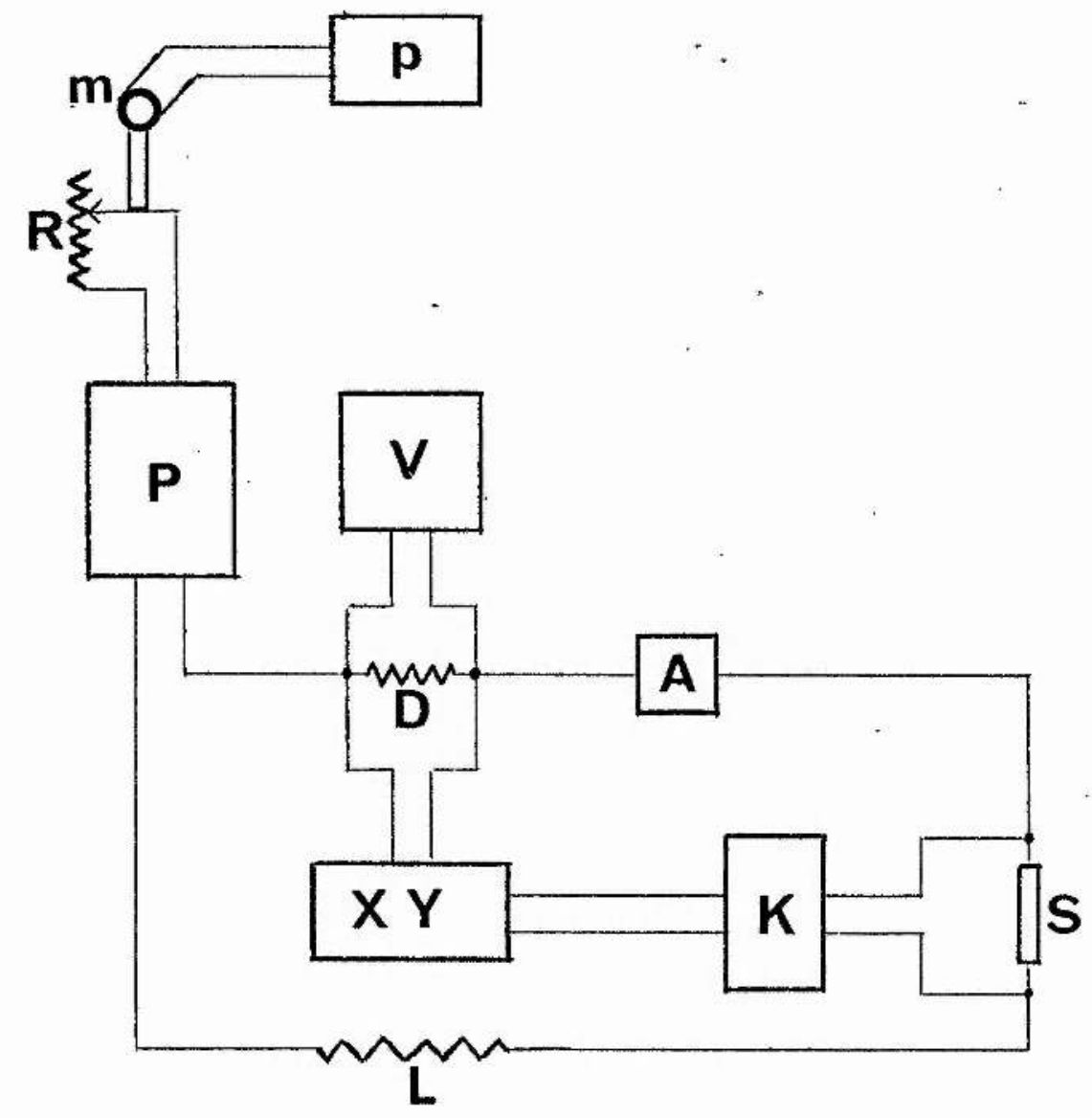

Fig. 3.1 Block diagram of the electrical circuit.

was achieved by using a small variable speed d. c. motor m to drive the potentiometer, with an appropriate gear system. The speed of this motor and hence the rate of change of the current $i$ in the main circuit could be controlled by regulating the output voltage of a laboratory power supply $p$ which was used to power the motor $m$. The rate of change of current could be varied between about $50 \mathrm{~mA} / \mathrm{mt}$. and $3 \mathrm{~A} / \mathrm{mt}$.

The main circuit was composed of the following other elements:

(i) $D$ is an oil-immersed, four terminal standard resistor of nominal value 1 ohm and rated to carry upto 3 Amps. with a quoted accuracy of $0.001 \%$. It served two purposes:

(a) The value of the current could be determined accurately by measuring the: voltage across $D$ with a d. c. potentiometer V. This enabled the current to be determined to $\pm 0.25 \mathrm{~mA}$ and was used to calibrate the scale of the Beckman potentiometer in terms of current.

(b) The potential across $D$ was fed into the $X$ channel of an $X-Y$ recorder $X Y$ 
thus enabling the value of the current to be plotted along the $X$ axis. (ii) A is an Avometer which, used on its current ranges, provided a rough indication of the current.

(iii) S represents the specimen of superconducting wire and will be described below. The potential across $S$ was fed into (iv) K, a low noise d. c. amplifier with an adjustable gain of upto 108. The model used was a Keithley 149 milli-microvoltmeter which, on its most sensitive range, gave a full scale deflection for 0.1 microvolt. The accuracy was within $2 \%$ of full scale on all ranges. The input shorted noise level was quoted at less than 3 nanovolts peak to peak and, in practice, was always found to be below 5 nanovolts peak to peak. The Keithley Model 149 acting as an amplifier gave a d. c. output (10 volts for full-scale deflection) and this was fed into the $Y$ channel of

(v) the X-Y recorder XY. As indicated previously, the potential across the standard 1 ohm resistor was fed into the $X$ channel of this recorder; thus the recorder gave us a continuous plot of V-I for the specimen involved. The voltages fed into the recorder being in the ranges 0 to 3 volts and 0 to 10 volts, the recorder did not require sensitive amplifiers and the model used was a Moseley 7035.

(vi) I is a load resistor. The remaining elements of the main circuit had a total resistance of just over an ohm and the value of $L$ was chosen in such a way that the Power Supplies $P$ were delivering about two thirds of their total available power under conditions of maximum current,

\section{3 THE CRYOGENIC SET-UP:}

Cryogenic experiments are fairly common nowadays and, as our experiment was a simple one and did not require any special techniques, the apparatus used will be described briefly.

Fig. 3.2 shows a sketch of the experimental set-up used. H is the Helium dewar made from Monax glass which is attached with an 'O'ring seal to the 


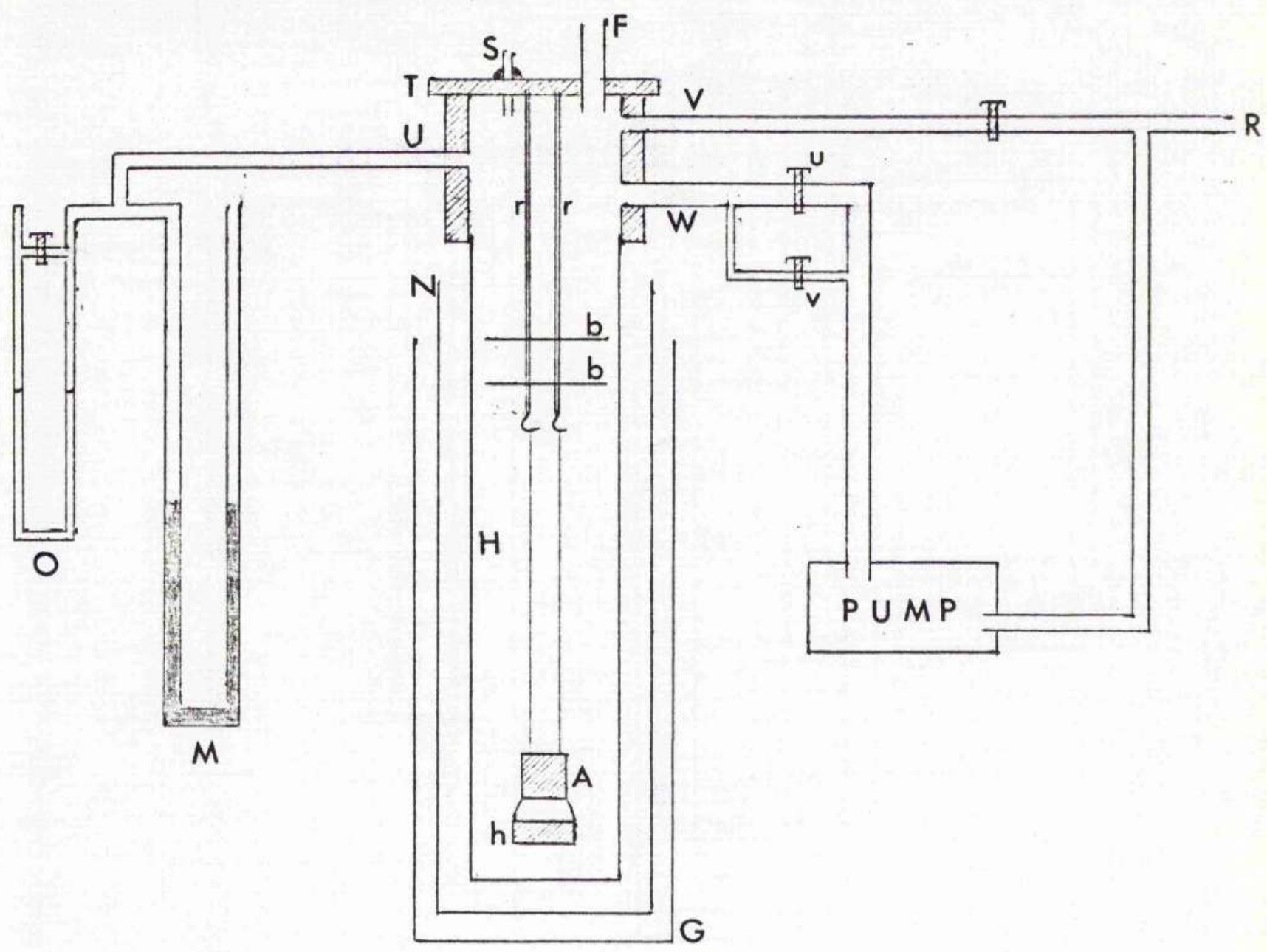

Fig. 3.2 Schematic of the cryogenic set-up.

cryostat neck and sits inside the liquid nitrogen dewar N. The latter is surrounded by a Mumetal cylinder G, open only at the top, whose dimensions are such that the lower part of the helium dewar (upto a height of about $10 \mathrm{cms}$. from the bottom) is screened from the earth's magnetic field. It was found that the shielding was about $98 \%$ effective.

The cryostat neck has three openings. $U$ is a quarter inch copper tube connecting the helium dewar to a mercury manometer $M$ and an oil manometer 0 . The mercury manometer together with the reading of a Fortin's barometer gave the absolute pressure of the system at any instant. With the help of a cathetometer the wide bore mercury manometer could be read to an accuracy of of 0.05 torr. During the latter part of the work a Van Mal-Akerboom (1968) type single-reading manometer was used. The oil manometer was filled with butyl phtalate and was 
particularly useful for monitoring changes of pressure. Another quarter inch line $\mathrm{V}$ connected the cryostat to the helium gas recovery line $\mathrm{R}$. $\mathrm{W}$ is a one inch line connecting the cryostat via valve $u$ to a rotary pump whose exhaust' could, when the system was pumping on helium, be connected to the helium gas recovery line R. The big vaflve u could be bypassed by a quarter inch line containing a sixteen turn needle valve $v$ and this provided a very fine control of the pumping rate. By monitoring the pressure of the system on the oil manometer and/or the mercury manometer with the help of a cathetometer and by using the needle valve $v$, the pressure could be kept constant to within about $\pm 0.5 \mathrm{~mm}$ on the oil manometer which, in the relevant range of pressure, is equivalent to keeping the temperature constant to within \pm 0.25 millidegrees. During the later stages of the work the use of a Cartesian Nanostat made it easier to control the temperature in the system.

The cryostat top plate $T$ has an opening $F$ for the liquid helium transfer siphon and several glass-metal seals $S$ for taking electrical leads into the cryostat. These were of the hollow type and copper leads were passed through them and soldered on both sides. Thus the potential leads to the specinen were all copper leads thereby reducing thermal noise. Seals were required for two voltage and two current leads to the specimen, two current leads to a carbon resistor heater $\mathrm{h}$ and an earth point. The carbon resistor had a nominal room temperature resistance of $10 \mathrm{ohms}$ and was fed from the power supply p (Fig.3.1). It was used to raise the temperature of the helium bath if required and also to help boil off excess helium at the end of an experimental run.

Three itainless steel tubes $r$ soldered to the base of the top plate helped support two copper discs b which acted as heat shields. The copper plates had concentric circular holes cut in them.to allow the helium transfer siphon to pass through. At their lower ends the tubes $r$ terminated in hooks from which the specimen holder A was suspended by nieans of nylon thread.

The specimen holder A (see plate I) consisted of a cylindrical former made 
of tufnol, a bakelite, of radius about one inch so that it just slipped comfortably into the helium dewar and about $2 \frac{1}{2}$ inches long. The cylinder had circumferential ruts cut into it so that the specimen could be laid in one of these ruts, without completing a full circle. In this way it was possible to mount a longer specimen (upto about $6 \mathrm{cms}$. in length) at the same horizontal level than would be possible with a straight specimen. When specimens were superconducting wires of diameter of the order of $1.0 \mathrm{~mm}$. or larger, the normal resistance of the specimens were sufficiently small that the noise (mainly thermal) voltage became significant enough to make the experiment unsatisfactory. For such wires longer specimens were used and these were wound round the former as shown in Fig. 3.3 so that (a) the winding was non-inductive, and

(b) the magnetic fields due to neighbouring portions of the wire were self-cancelling. Such a specimen would be spread over a height

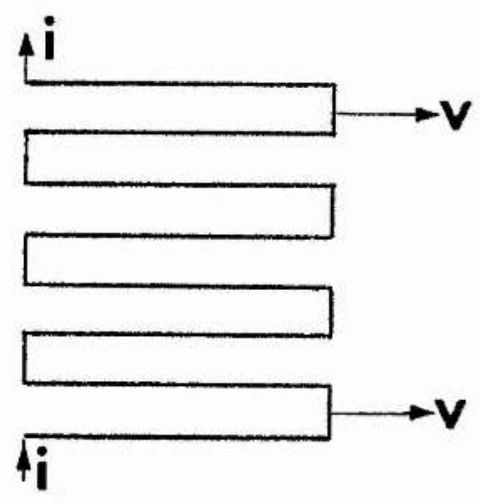
of about 2 to $3 \mathrm{cms}$. in the helium so that the temperature of the specimen may vary by about a millidegree, and the resistance jump is slightly less sharp than would otherwise be the case. However by comparing results obtained with long and short specimens of a thin wire, it was found that the value of the resistance jump $\rho$ itself was the same (to within experimental spread) in both cases. Hence for our purposes the slight spread in the transition was not an important factor.

Enamelled copper wires were used for the current and voltage leads and optimum guages were calculated following Rose-Innes(1959) to minimize the heat input due to thermal conductivity and joule heating. The leads were soldered on to the specimen with Woods Metal solder using a low temperature soldering iron. A $5 \mathrm{Kohm}, 25$ watts potentiometer was connected in series with the soldering iron to regulate its temperature. 


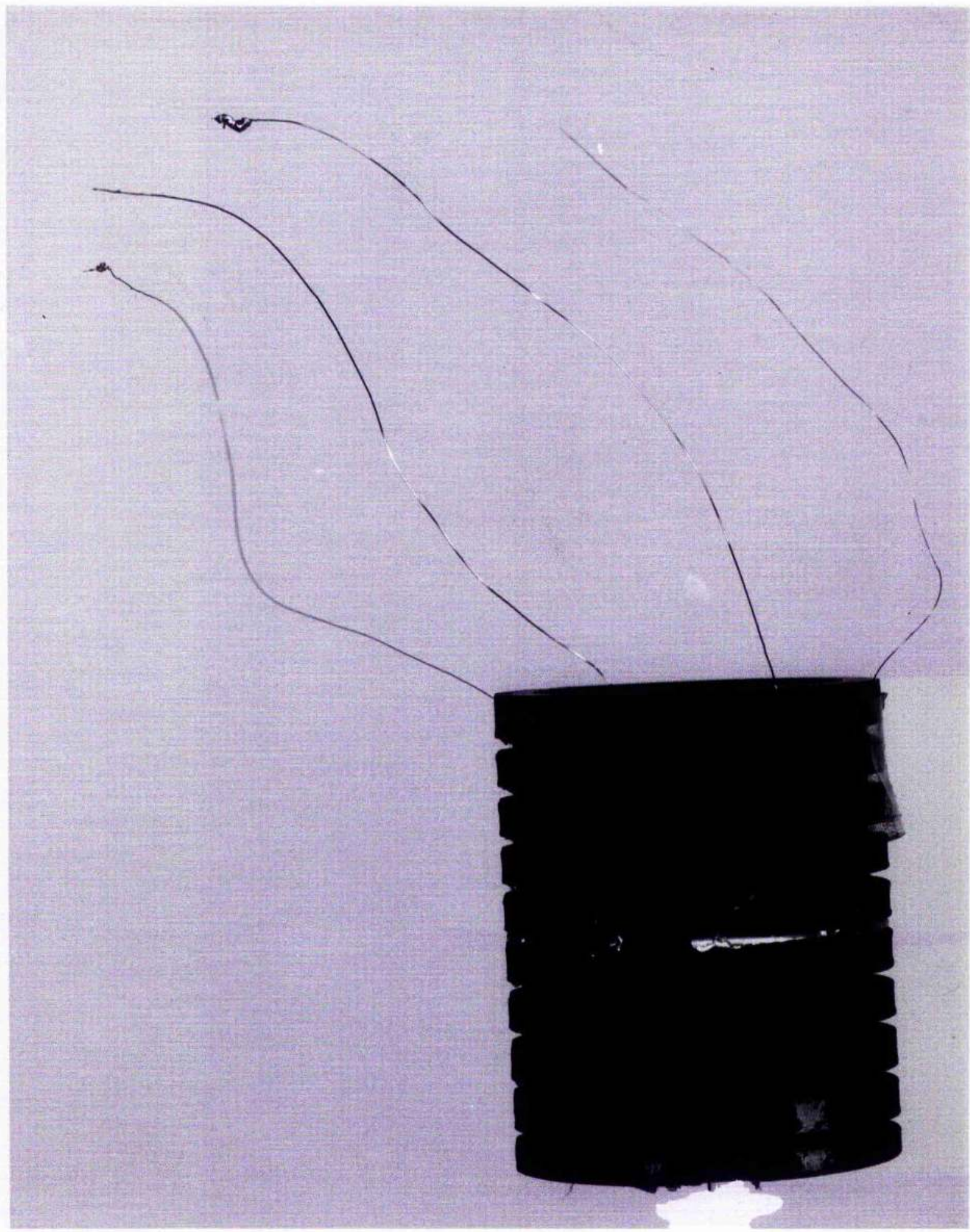

PLATE I. The Specimen Holder 


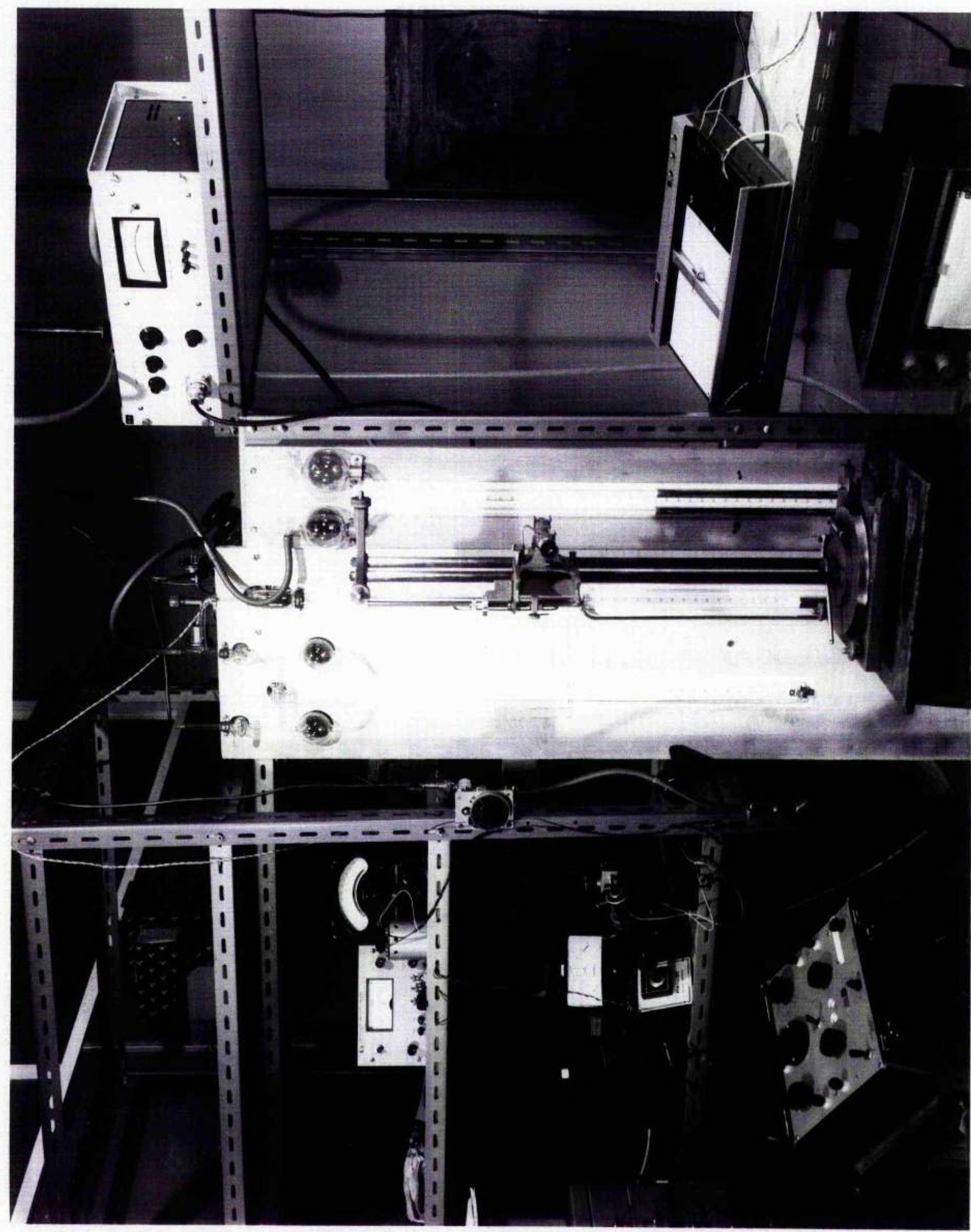

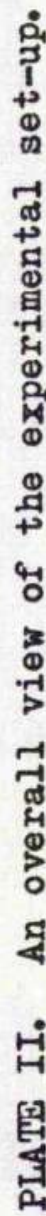




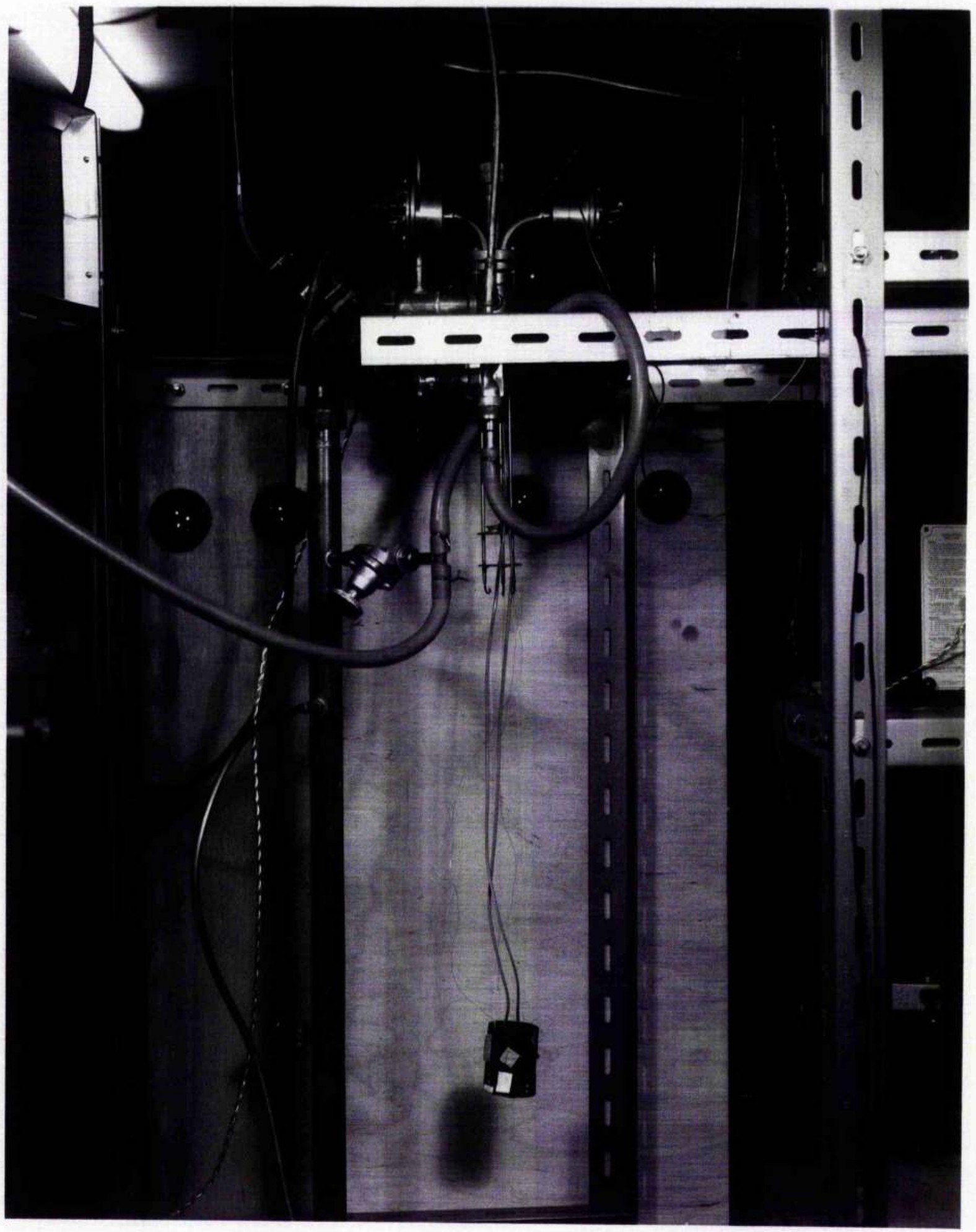

PLATE III. Rear view of experimental set-up with the dewars removed and the specimen holder in place. 
An overall view of the experimental set-up is shown in Plate 2 and in 2 Plate 3 the specimen holder is shown in situ with the dewar removed.

\section{4 THE SPECINENS:}

The experimental investigation was carried out mainly on Indium specimens. We were primarily interested in obtaining a few accurate observations (particularly on the resistance jump $\rho$ ) for very pure metal. On the basis of theory and previous work there was no reason to suppose that the transition differed fundamentally as between different metals. On several counts it was particularly convenient to work with Indium:

(a) it is easy to obtain very pure Indium.

(b) with a $\kappa$ value of about 0.11 Indium is a strongly Type-I superconductor. (c) it is relatively easy to extrude Indium wires.

(d) Indium anneals at room temperature.

(e) Indium has a critical temperature of $3.407^{\circ} \mathrm{K}$. which is very convenient for experimental purposes.

Pure Indium was obtained from two sources: Johnson Matthey and Co., London, whose Indium had a quoted purity of $99.9995 \%$, and Consolidated Mining Co. (Cominco) of Montreal, Canada, who quoted the purity of their Indium at 99. $9999 \%$ Resistance measurements on the Indium specimens at room temperature and at $4.2^{\circ} \mathrm{K}$ showed the Cominco Indium to be much the purer and most of the experiments were done with their Indium. It was interesting to note that the purer Indium vas much stickier too. This stickiness constitutes the main problem in extruding Indium wires, as the Indium tends to creep up the sides of the extruder piston and jam it. With this in view, an extruder was made out of tool steol with the piston made to fit the cylinder to a fine degree of tolerance and the whole was heat treated in an atmosphere of hydrogen. The piston head was recessed and this helped to reduce the creep of Indium up the cylinder wall during extrusion. The extrusions were carried out at room temperature with the help of an automati hydraulic press; by this means the wires drawn had their diameters constant to 
within $\pm 0.005 \mathrm{~mm}$.

Altogether seven Indium specimens were used with their diameters varying in the range $0.25 \mathrm{~mm}$. to $1.65 \mathrm{~mm}$. Additionally experiments were carried out with one specimen of Tin wire of $0.5 \mathrm{~mm}$. diameter and a specimen of thallium wire of $1.0 \mathrm{~mm}$. diameter. Both of these wires were obtained from Metals Research Ltd., Royston. Table 3.1 describes the specimen used; the purity given in column 4 is that quoted by the suppliers while column 5 gives the ratio $R_{4.2} / R_{293}$ of the resistivities of the specimens at the temperature of liquid helium at atmospheric pressure and at room temperature.

In the case of Indium I the current and voltage leads were connected close together

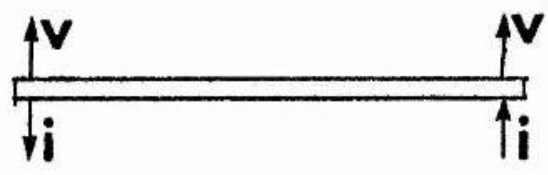
near the ends of the specimen as indicated in Fig. 3.4(a). However this set-up resulted in a tail in the resistance transition curve, i.e.

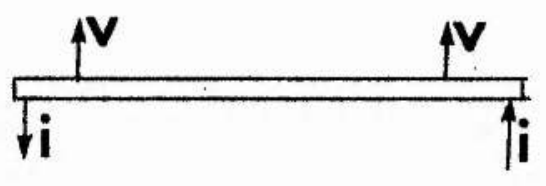
instead of a sharp jump in resistance when the current became critical, the resistance showed a slow increase even before $i_{c}$ was reached as may be seen in Figs. 3.5 and 3.8. In the case of Indium II the voltage leads were connected to the specimen at points about a $\mathrm{cm}$. away from where the current leads were soldered, as

Fi.g.3.4 Sketch showing the relative positions where the voltage and current leads werd attached to the specimen (a) for Indium $I$ and Thallium $I$ and (b) for all other specimens. shown in Fig. 3.4(b) and no 'tail' was obtained (see Figs.3.6 and 3.9). This can probably be explained as follows: The current leads being of copper, a nonsuperconducting metal, a certain amount of heat is generated in them by the passage of current. Consequently the ends of the specimen, where it makes contact with the current leads, will be slightly heated and will have a lower critical current than the rest of the specimen, thus giving rise to the 'tail' if the voltage leads are connected close to the current leads. For all specimens other than Indium I and Thallium I the current and voltage leads were 


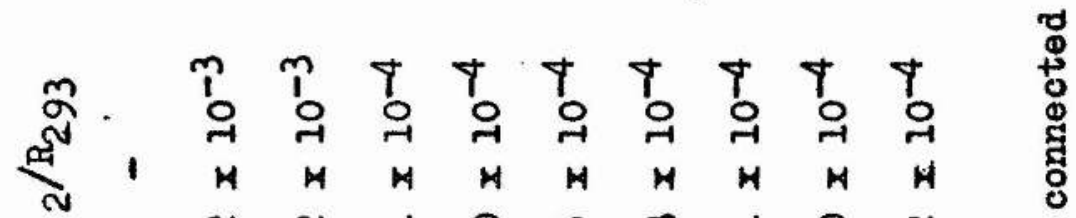

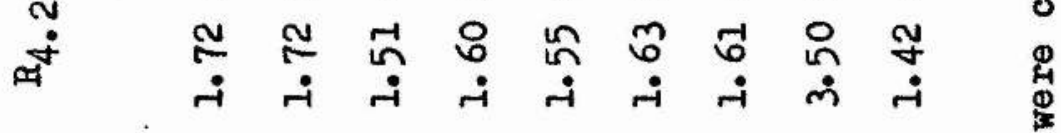

范

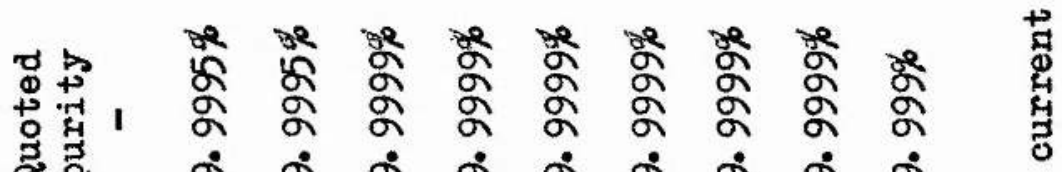
๙

苗

वु

게

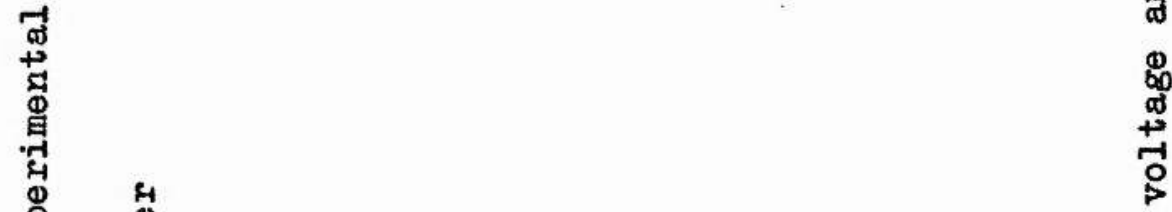

웠 영

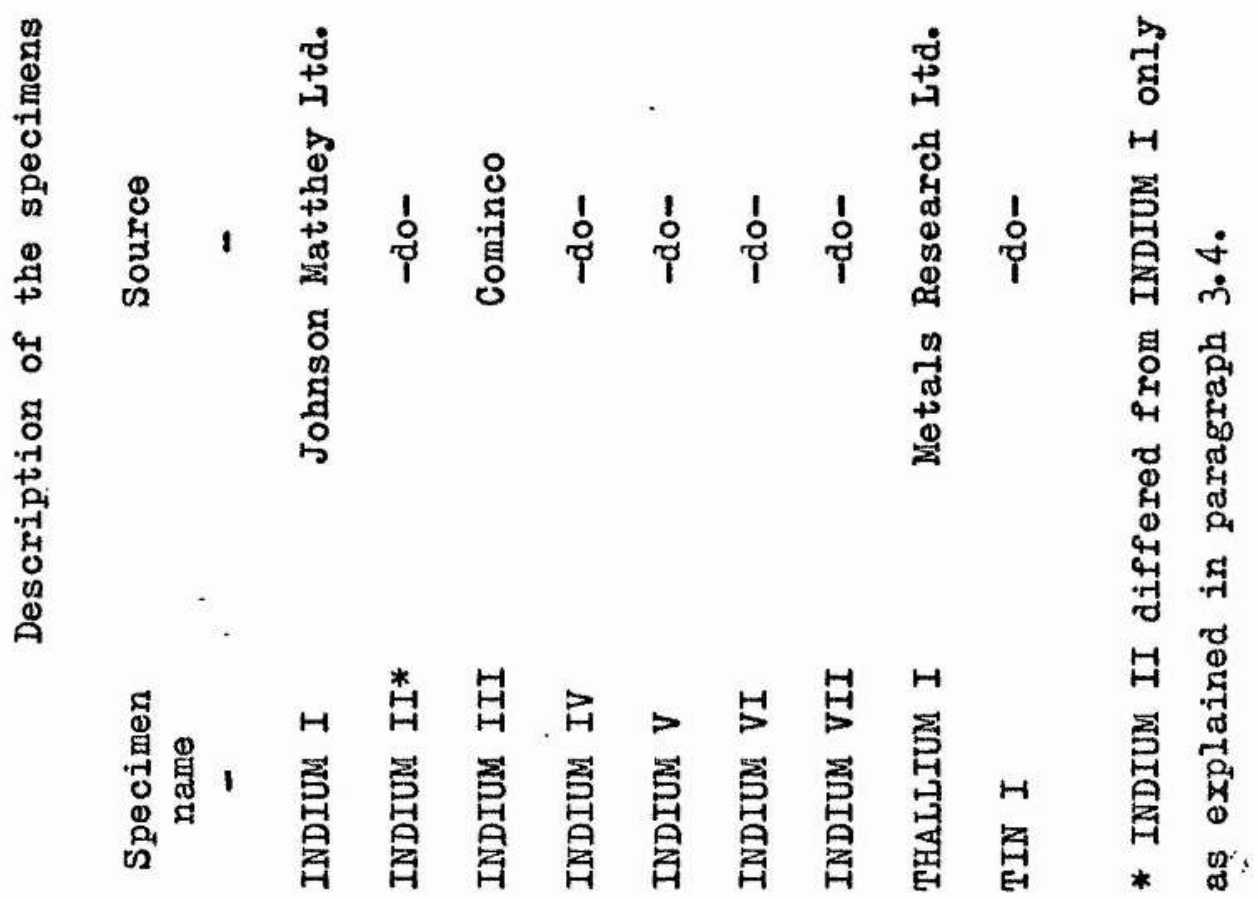


connected as shown in Fig. 3.4(b) with a minimum gap of $1.0 \mathrm{~cm}$. between the current and voltage connections.

\subsection{THE EXPERIMENTAL RESULTS:}

The output from any experiment consisted of an $X-Y$ recorder plot of the current-voltage characteristic for a specimen kept at a constant temperature. Three typical curves are shown in Figs. 3.5, 3.6 and 3.7 , and it can be seen from these continuous plots (except in Fig. 3.5 for reasons discussed in the previous paragraph) that when the current reaches its critical value the resistance jump is well defined. We thus obtain a more accurate value of $\rho$ than would be possible if discrete measurements of $(V, i)$ were made and a curve drawn to pass through them. Figs. 3.6 and 3.7 show that the resistance jump at $i=i_{c}$ is spread over a small current range; this is an experimental artefact due to the small but finite rate of change of current. By manually operating a very fine current control it was possible to eliminate the transition width and it was found that the values of $\rho$ obtained in this way lie within the experimental spread in the values of $\rho$ obtained from the continuous plots.

It is the resistance of a wire specimen rather than the voltage across it that is the basie quantity of interest to us. Accordingly our experimental results are presented in Figs. 3.8 to 3.15 in the form of curves showing the variation of the resistance ratio $R / R_{n}$ with current $i$. These have been obtained by conversion from the $V-i$ curves on a point by point basis and since the latter were continuous curves, it has been possible to use a large number of points at regions of large curvature.

The inherent noise level in the experiments was of the order of 20 nanovolts. As may be seen in Figs. 3.5 to 3.7 , there were occasional random excursions of the order of 100 nanovolts or less - if these occurred where the curvature was small, the curve was smoothed out, but when they happened near the sharp transition, the curve was rejected and the experiment was repeated if required. 
3. 11

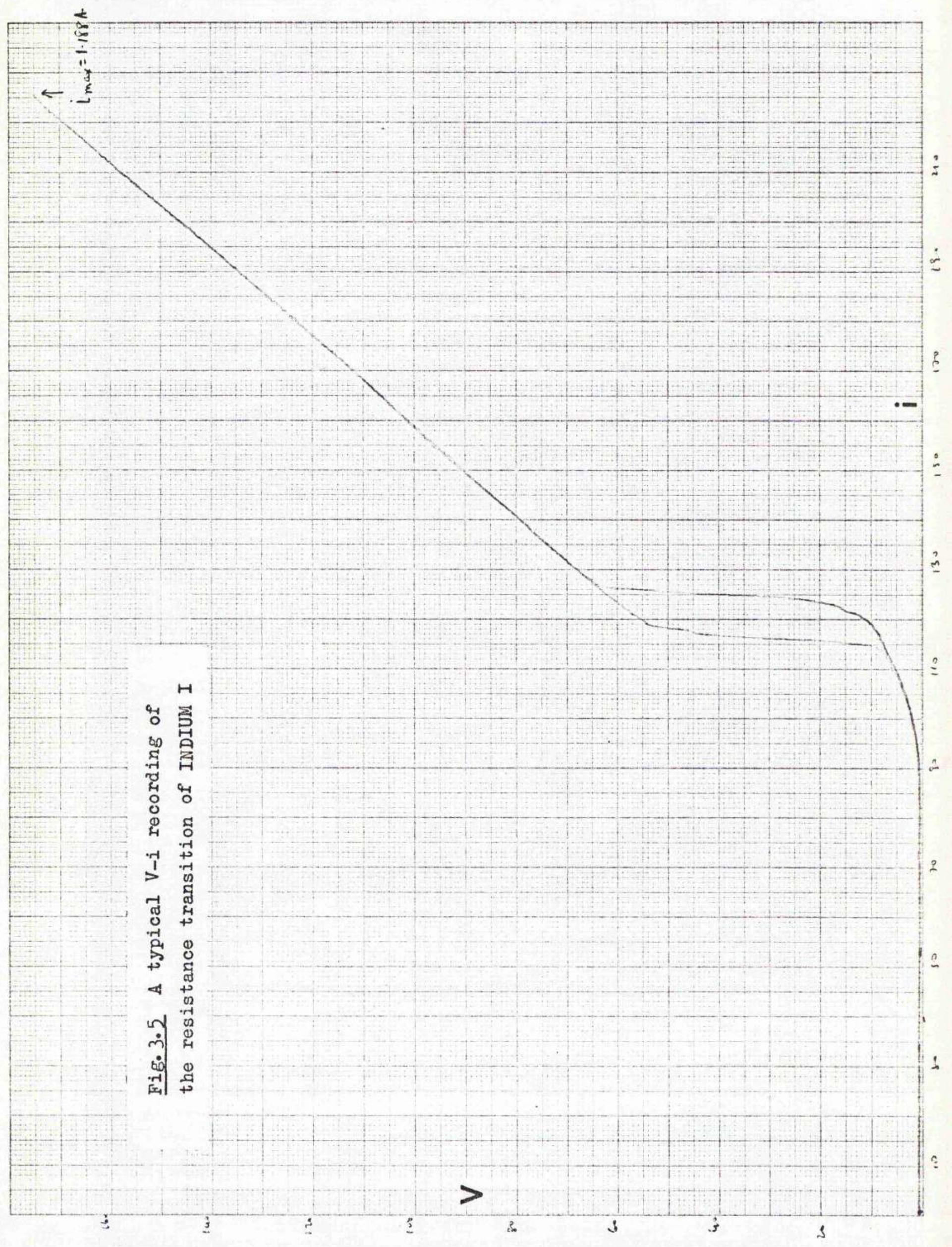


3.12

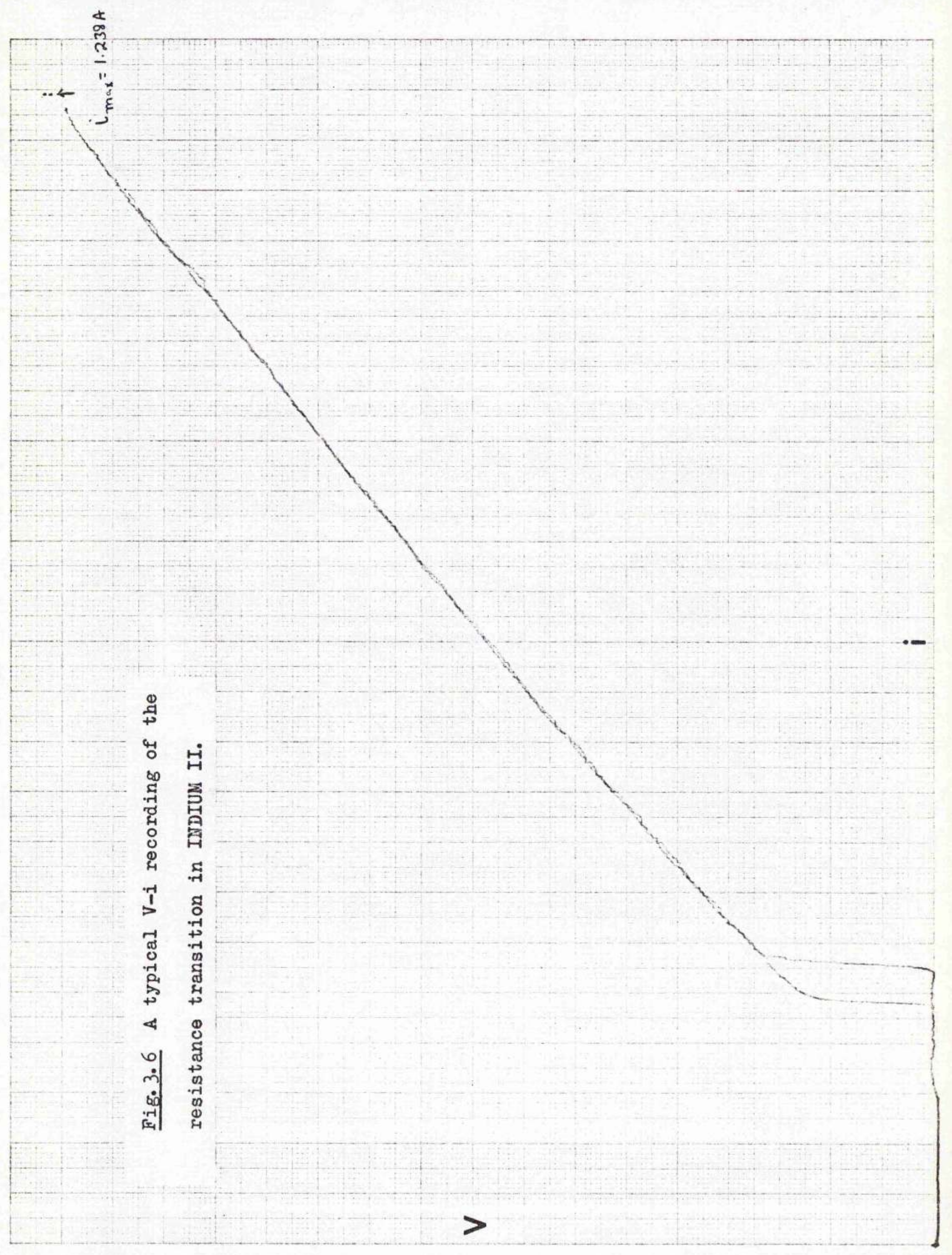




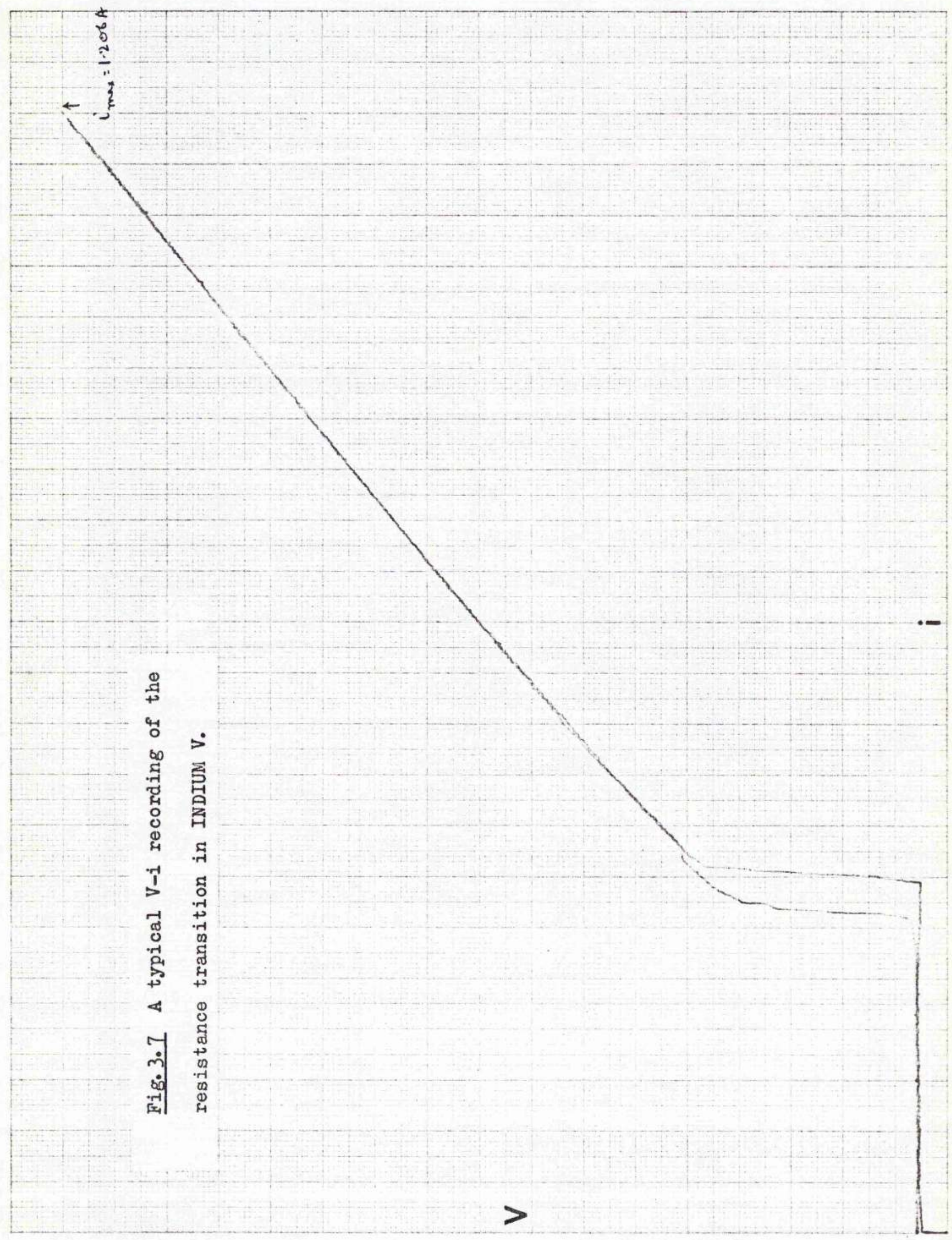




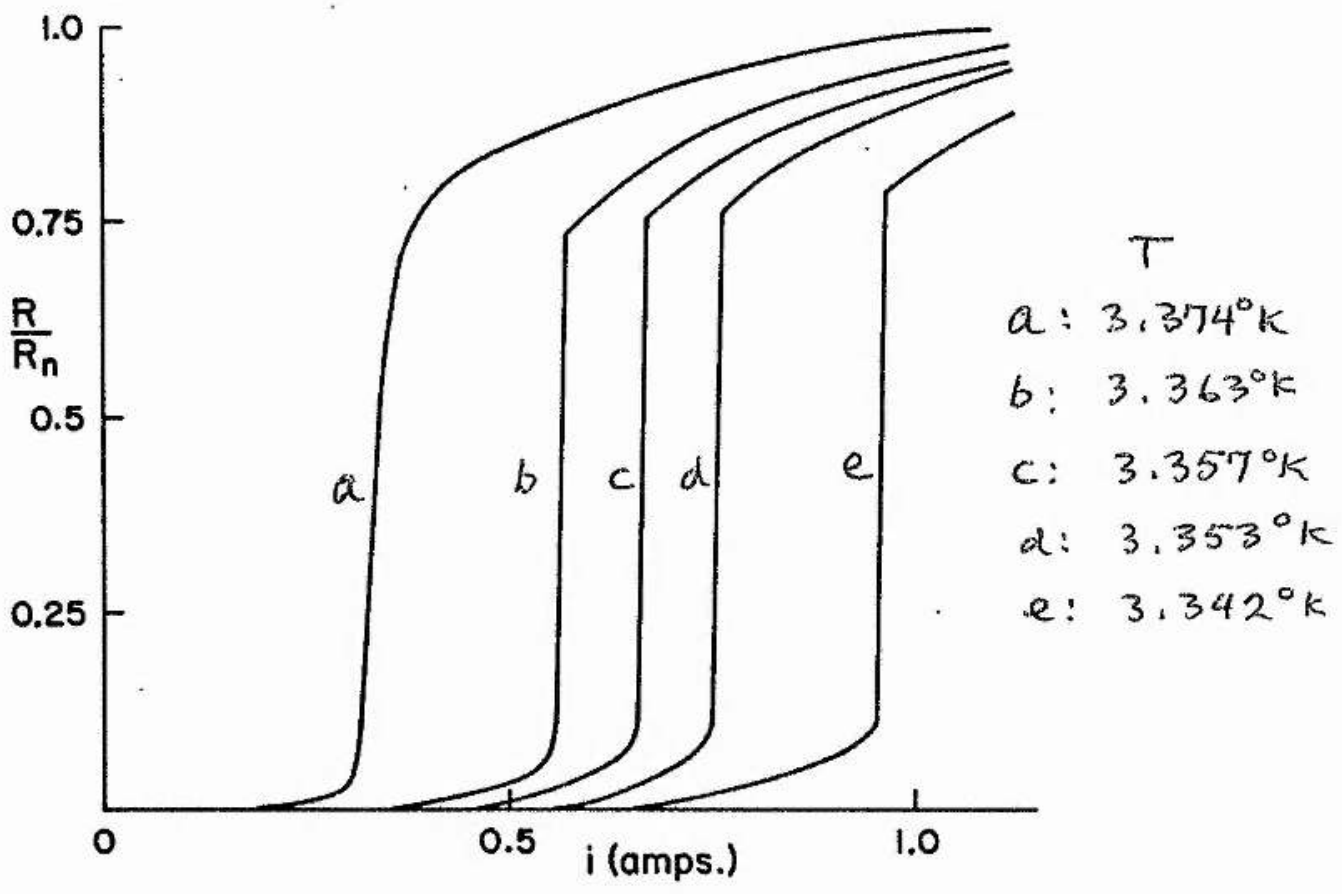

Fig. 3.8 The resistance ratio $R / R_{n}$ as a function of current $i$ for specimen INDIUM I $\left(R_{4.2} / R_{293}=1.72 \times 10^{-3}\right.$, wire diameter $=0.5 \mathrm{~mm}$.)

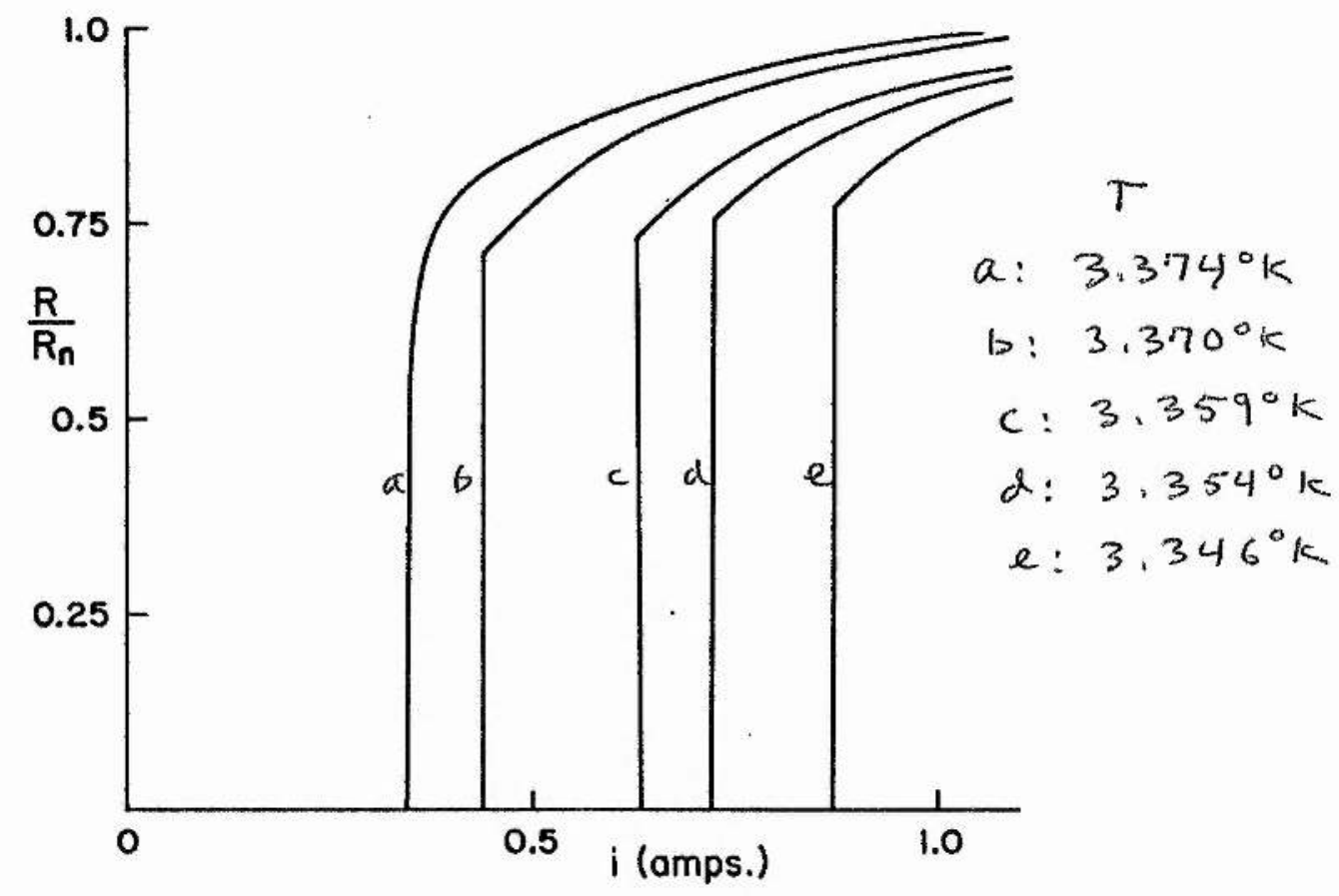

Fig. 3.9 The resistance ratio $R / R_{n}$ as a function of current $i$ for specimen INDIUM II $\left(R_{4.2} / R_{293}=1.72 \times 10^{-3}\right.$, wire diameter $=0.5 \mathrm{~mm}$ ) 


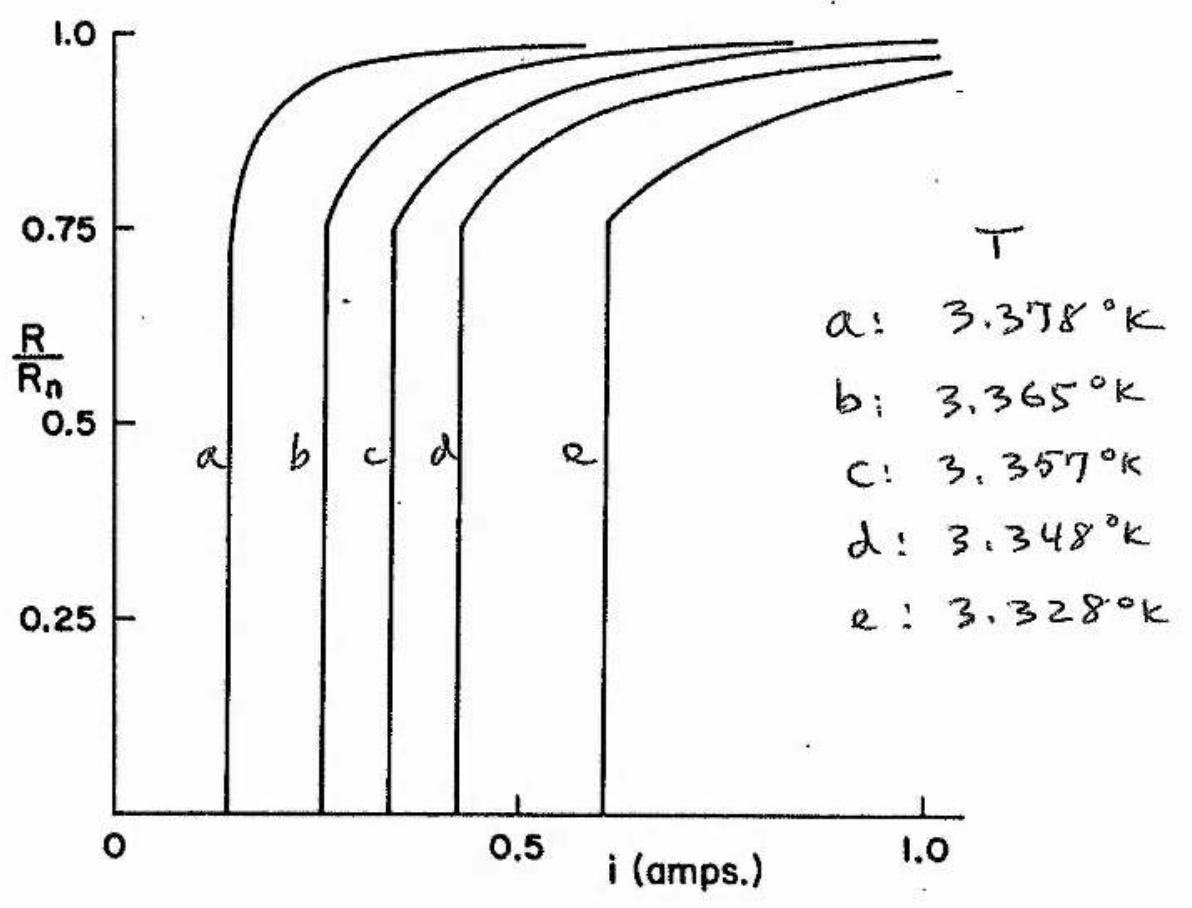

Fig. 3.10 The resistance ratio $R / R_{n}$ as a function of current $i$ for specimen INDIUM III $\left(R_{4.2} / R_{293}=1.51 \times 10^{-4}\right.$, wire diameter $=0.25 \mathrm{~mm}$.)

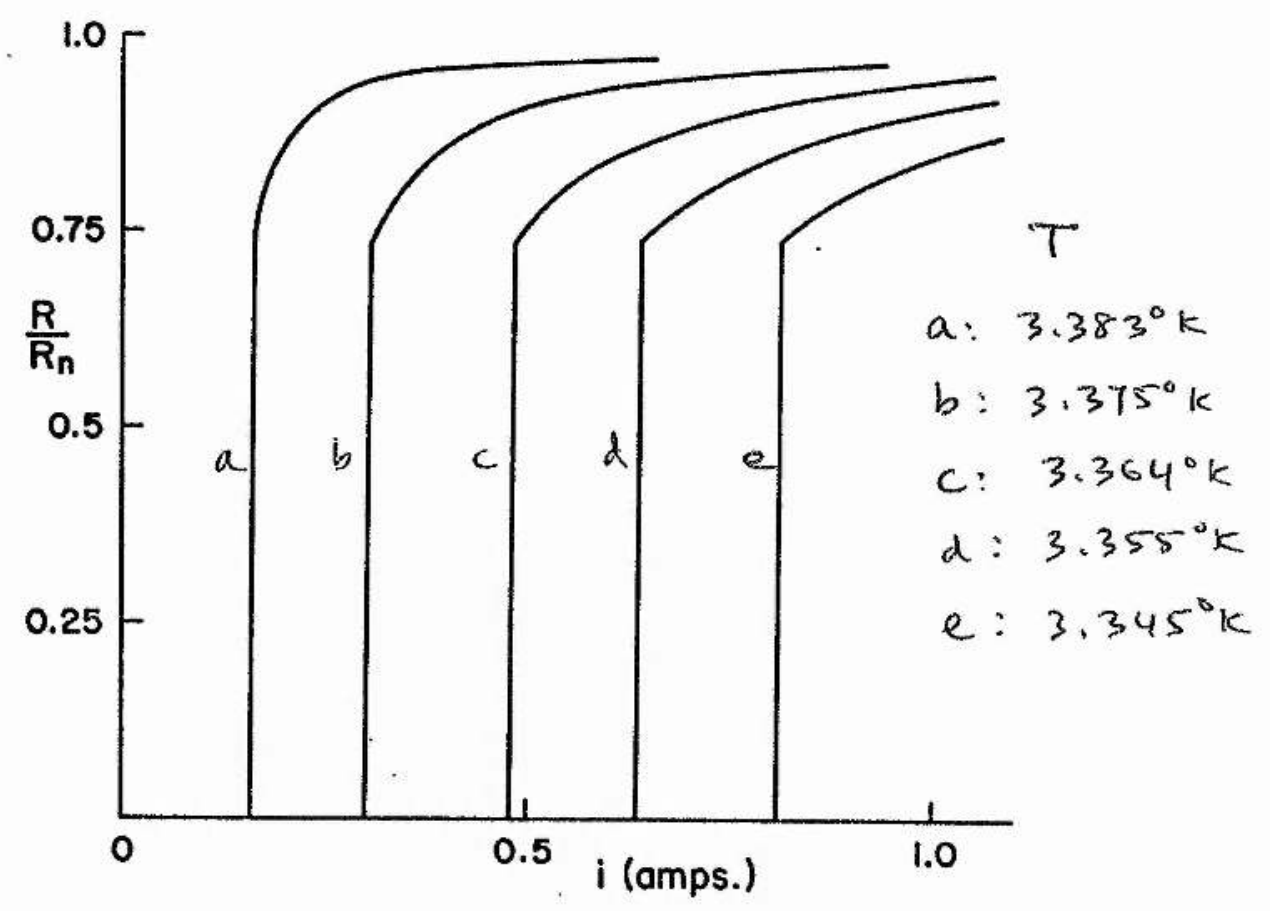

Fig. 3.1. The resistance ratio $R / R_{n}$ as a function of current $i$ for specimen INDIUM IV $\left(R_{4.2} / R_{293}=1.60 \times 10^{-4}\right.$, wire diameter $=0.45 \mathrm{~mm}$ ) 


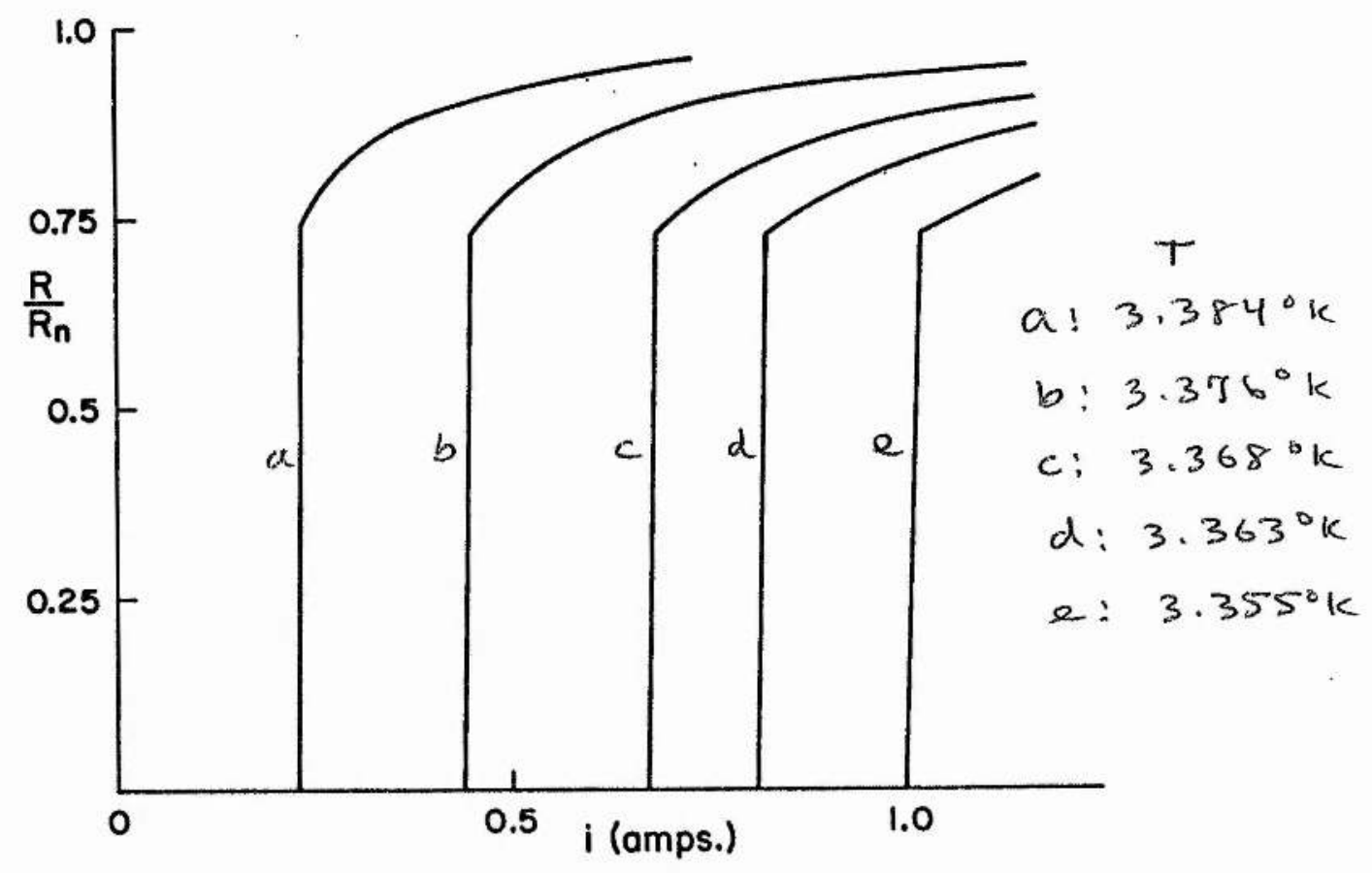

Fig. 3.12 The resistance ratio $R / R_{n}$ as a function of current $i$ for specimen INDIUM $v\left(R_{4.2} / R_{293}=1.55 \times 10^{-4}\right.$, wire diameter $=0.72 \mathrm{~mm}$ )

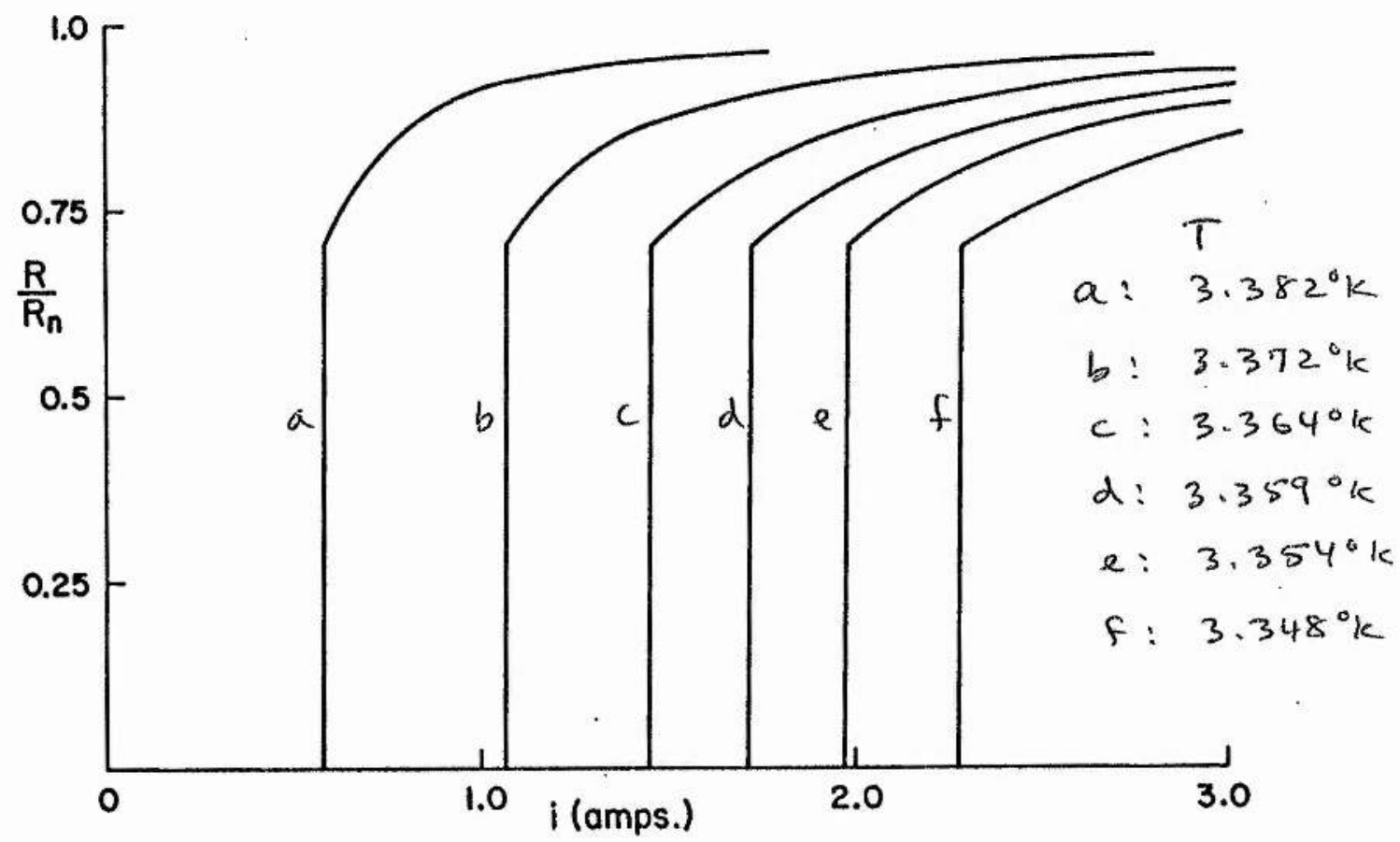

Fig. 3.13 The resistance ratio $R / R_{n}$ as a function of current $i$ for specimen INDIUM VI $\left(R_{4.2} R_{293}=1.63 \times 10^{-4}\right.$, wire diameter $=1.40 \mathrm{~mm}$.) 


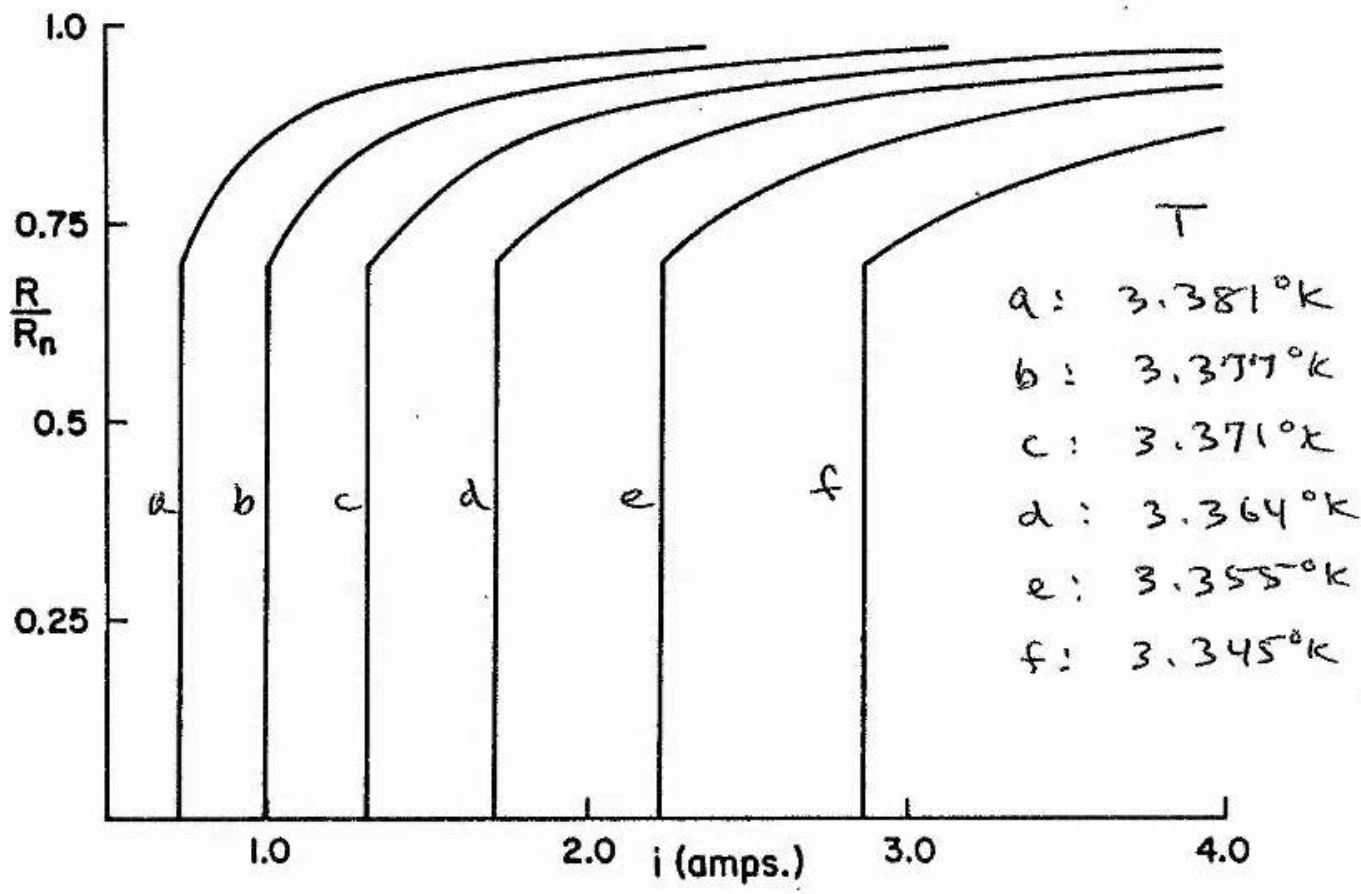

Fig. 3.14 The resistance ratio $R / R_{n}$ as a function of current $i$ for specimen INDIUM VII $\left(R_{4.2} / R_{293}=1.61 \times 10^{-4}\right.$, wire diameter $=3.65 \mathrm{~mm}$ )

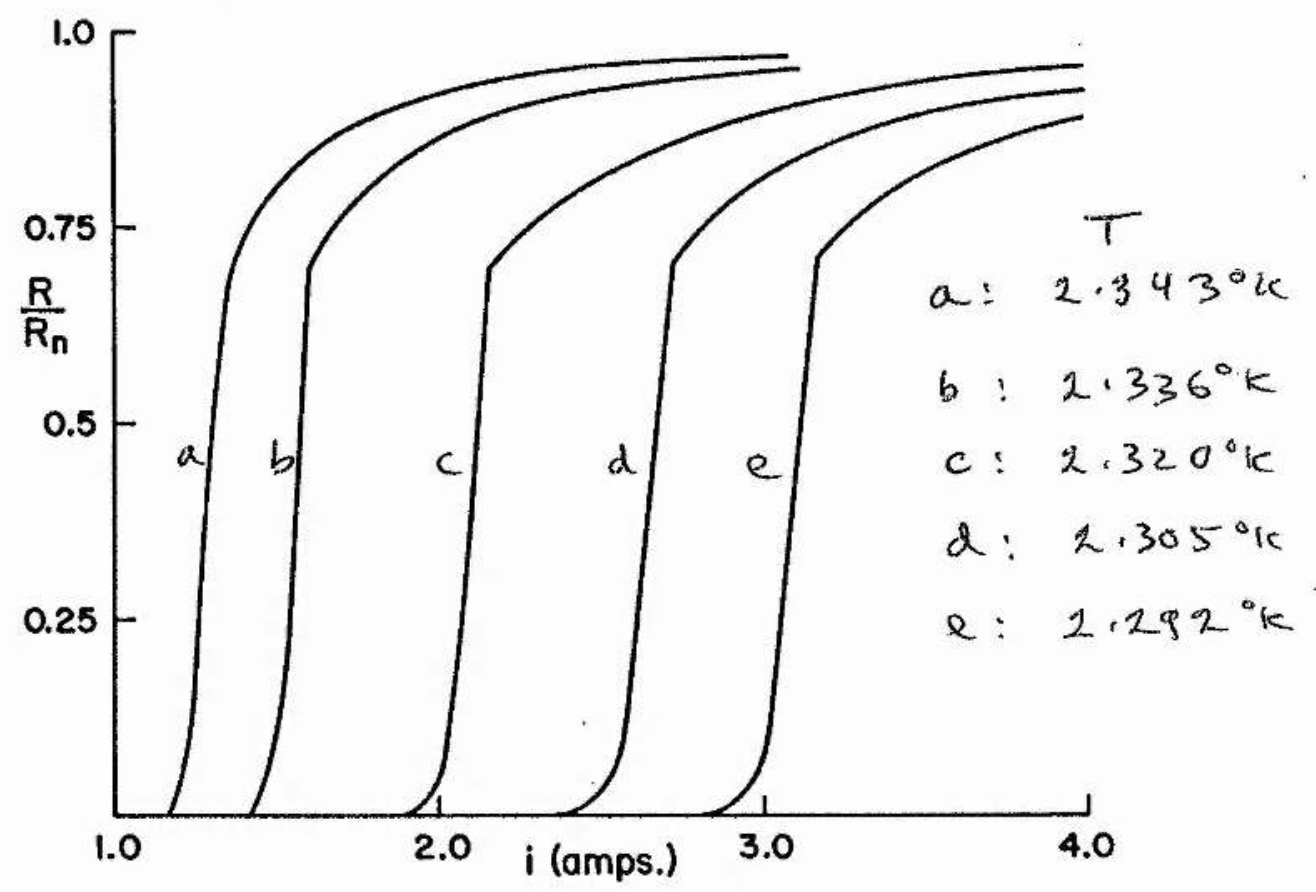

Fig. 3.15 The resistance ratio $\mathrm{R} / \mathrm{R}_{\mathrm{n}}$ as a function of current $i$ for specimen THALLIUM I $\left(R_{4.2} / R_{293}=3.5 \times 10^{-4}\right.$, wire diameter $=1.0 \mathrm{~mm}$ ) 


\subsection{DISCUSSION: :}

We shall now discuss the conclusions that may be drawn from our experimental results.

With certain exceptions which will be discussed below, the basic currentinduced transition in superconducting wires may be described as follows: for values of current less than a certain critical value $i_{c}$ the wire is fully superconducting; at the critical current resistance suddenly appears and jumps to a considerable fraction $\rho$ of the fully normal resistance; as the current is increased further, the resistance increases gradually until it asymptotically, reaches the fully normal resistance at a current of about $4 i_{c}$ for very pure metals.

For very low current transitions, the resistance jump at $i_{c}$ is not sharply defined but becomes smoothed out as may be seen in Figs. 3.8, 3.9, 3.10 and 3.11 This has been observed before (D.C. Baird, private communication) but is not yet properly understood. However it may be pointed out that a comparision between the results for INDIUM II and INDIUN IV shows that the effect of making a specimen impure is to increase the range of current over which the resistance jump is not sharply defined.

Leaving aside these 'low-current' transitions, our results (as well as the results of other workers) indicate that the value of $p$ varies from 0.69 upwards. The exact value seems to depend on temperature (i.e. the critical current), the diameter of the wire and on the purity. For example, Fig.3.16 shows the variation of the resistance jump $p$ with critical current for two cases: INDIUM II and INDIUM IV. In the case of INDIUM II the value of $\rho$ goes up as the critical current increases. However in the case of INDIUM IV, which is a purer specimen, the value of $\rho$ remains constant (within the limits of experimental accuracy) for values of $i_{c}$ less than about 0.9 Amps. and then begins to increase as the oritical current is increased further. In the case of INDIUM VJJ, which has the same purity as INDIUM IV but is a much thicker wire, 


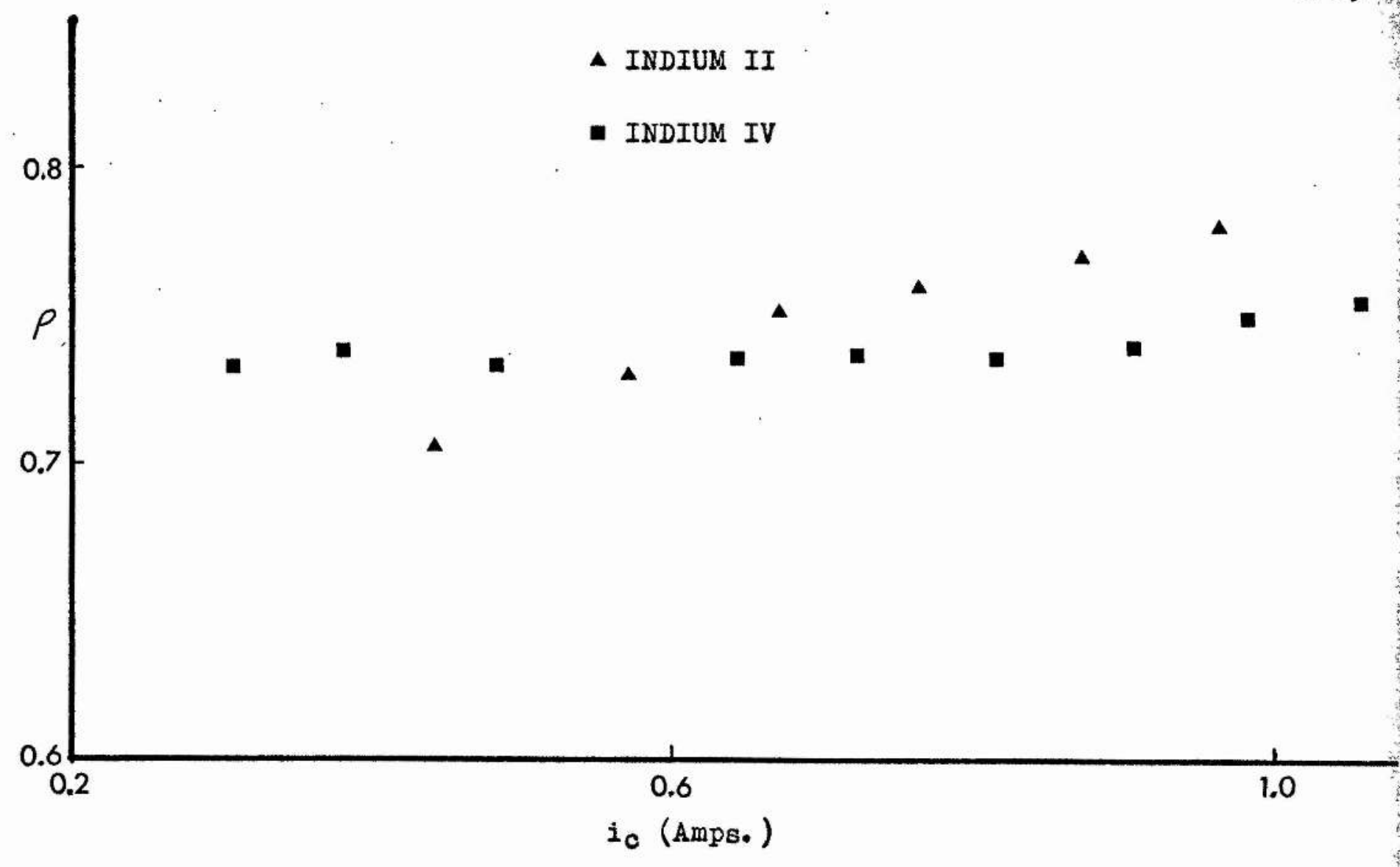

Fig. 3.16 Variation of the resistance jump (at $i=i_{c}$ ) with critical current for INDIUM II and INDIUM IV specimens.

the value of $\rho$ remains constant upto at least $i_{c}=2.5$ Amps. (see Fig. 3. 14\%). These observations suggest that the value of $\rho$ is independent of the critical current as long as the latter is below a certain limiting value; this limiting value becomes smaller as the wire becomes thinner or less pure. When the critical current is greater then the limiting value $i_{l}, \rho$ increases as $i_{c}$ goes up and the increase is larger for less pure specimens.

Fig. 3.17 shows the variation of the resistance jump $\rho$ with diameter of wire for very pure Indium where the point corresponding to a wire diameter of $3 \mathrm{~mm}$. has been taken from the work of Freud et al (1968). It appears that $\rho$ has a value of about 0.695 for thick, pure Indium wires but rises gradually as the wire diameter is reduced below about $1.5 \mathrm{~mm}$. Scott (1948) and Meissner and Zdanis (1958) amongst others had previously studied the variation of as a function of wire diameter. The present work along with that of Freud and his collaborators confirms that even for thick, pure wires the value of 


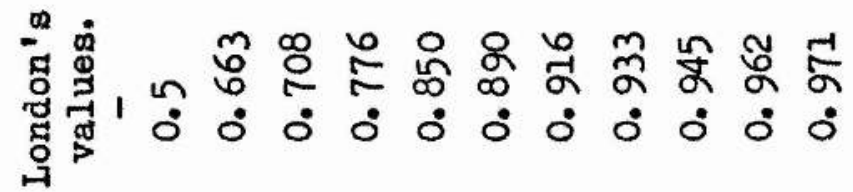

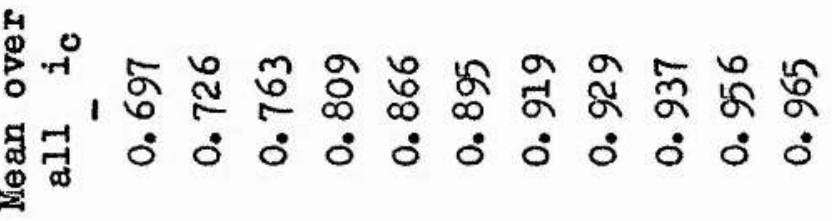

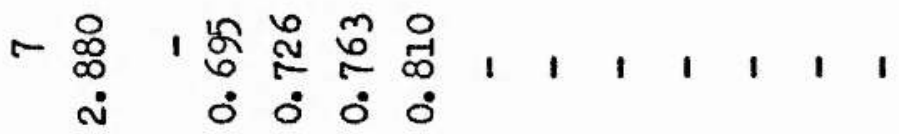

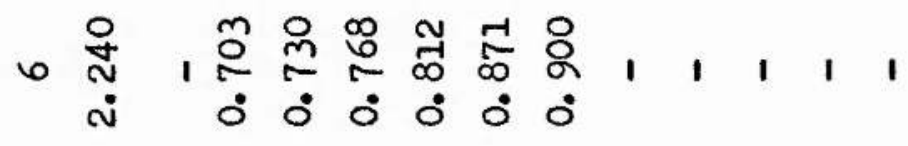

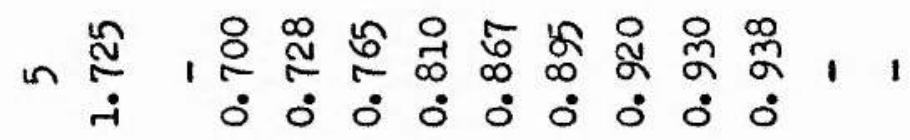

$$
\begin{aligned}
& + \text { ్ㅣ, }
\end{aligned}
$$

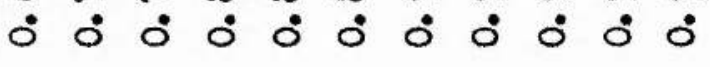

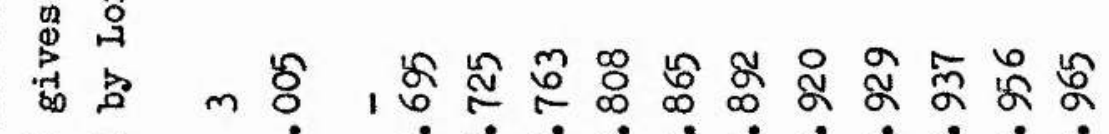

$$
\begin{aligned}
& \infty \begin{array}{lllllllllllll}
\infty & -1 & 0 & 0 & 0 & 0 & 0 & 0 & 0 & 0 & 0 & 0 & 0
\end{array}
\end{aligned}
$$

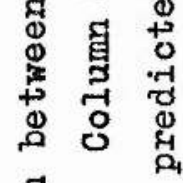

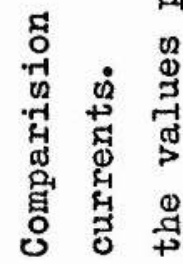

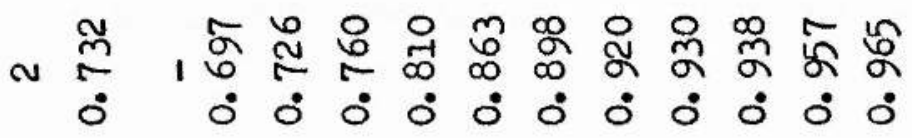

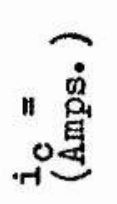

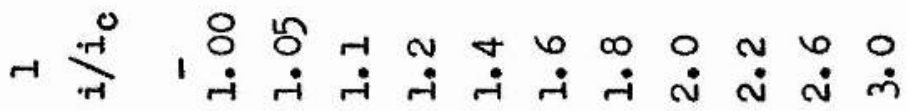




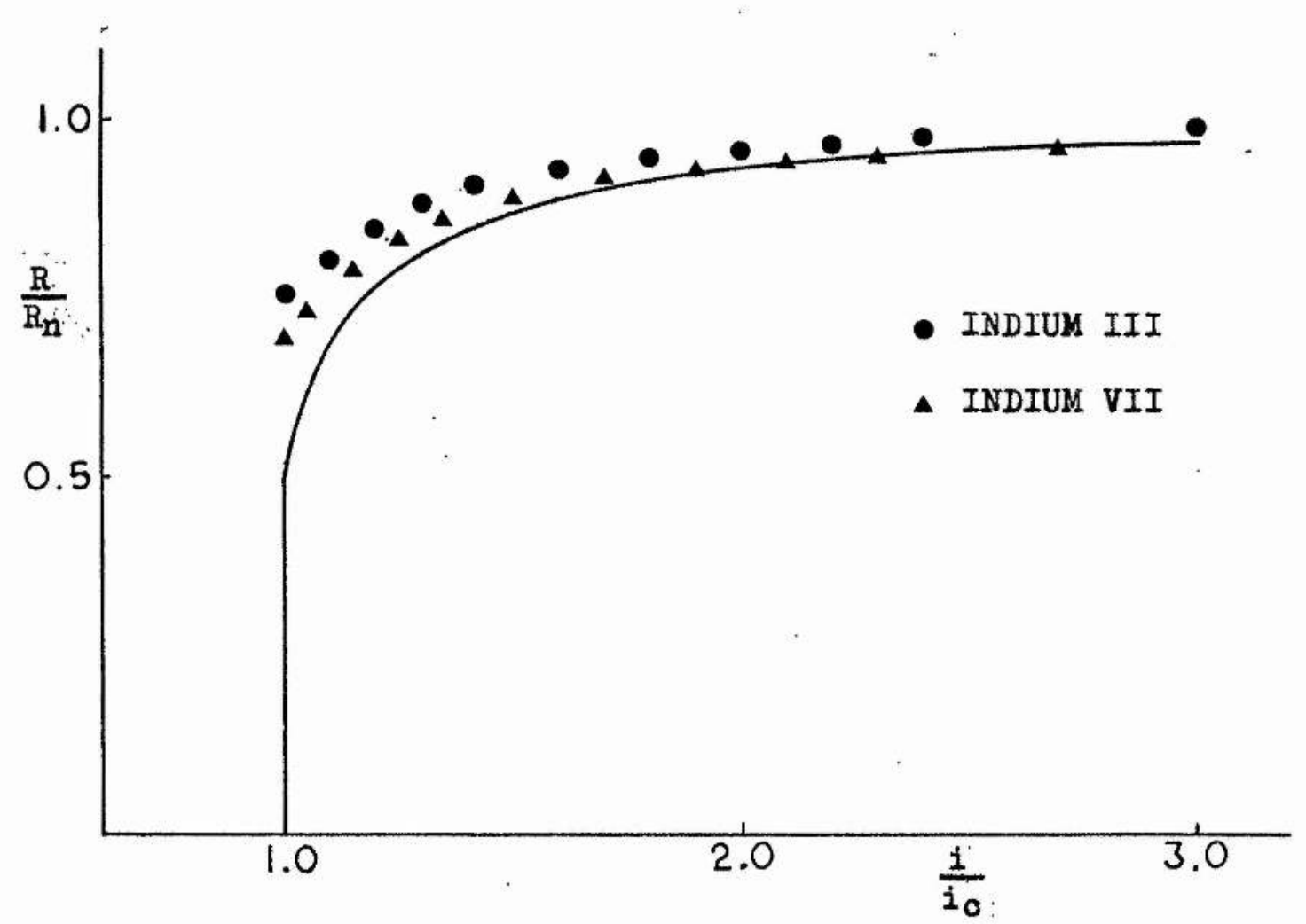

Fig. 3.18 Experimental values of the resistance transition of INDIUM III and INDIUM VII specimens compared with the theoretical curve predicted by London.

discussion has indicated that (a) increasing the wire thickness any further has negligible effect, and (b) increasing the impurity content only increases the value of the resistance for any value of $i / i_{0^{*}}$. It is therefore clear that the predictions of London's model do not agree quantitatively with our experimental results.

Our discussion so far has been restricted to Indium specimens because the availability of results on wires of different diameters and different purities enabled us to draw conclusions on the dependence of $\rho$ on these factors. However resistance transition experiments were also carried out with a Thallium wire of $1.0 \mathrm{~mm}$. diameter and a Tin wire of. $0.5 \mathrm{~mm}$. diameter. The results for Thallium are shown in Fig. 3.15: There was considerably more noise in the V-i curves obtained for Thalliumand the curves of Fig. 3.15 should only be taken as accurate to within 3\%; taking this into consideration, the Thallium curves fit in quite well vith the transitions obtained for Indium wires of different diameters, suggesting that, apart from some possible secondary effects, the 
basic resistance transition is independent of the Type-I superconductor used.

Our results for the Tin specimen were not very meaningful; no sharp transition was observed even for currents upto 3 Amps. as shown in Fig. 3.19. This was perhaps due to the specimen being an extruded sample and hence quite polycrystalline. De Haas and Voogd (1931) and Aziz and Baird (1959) have show that the presence of grain boundaries broadens the transition. The experiment was repeated after the tin wire was annealed for about twenty four hours at just below its melting point; this time the transition remained wide and seemed to proceed by more distinct jumps (see Fig.3.20) suggesting an increase in grain size, with each jump perhaps representing the transition of a single grain. Further annealing appeared to make little difference and in any case it was felt that the influence of grain size on the transition was a problem beyond the scope of this work. 


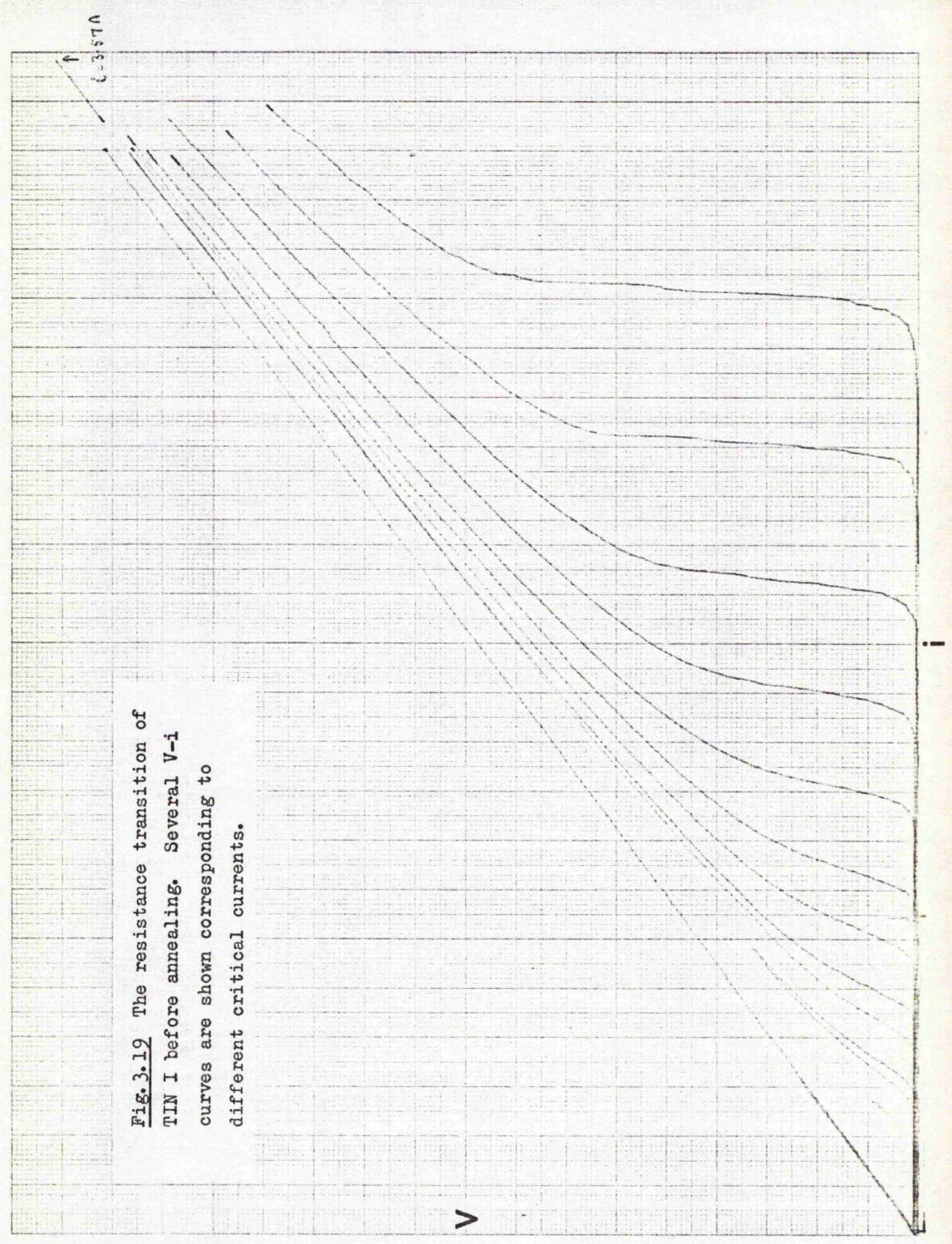




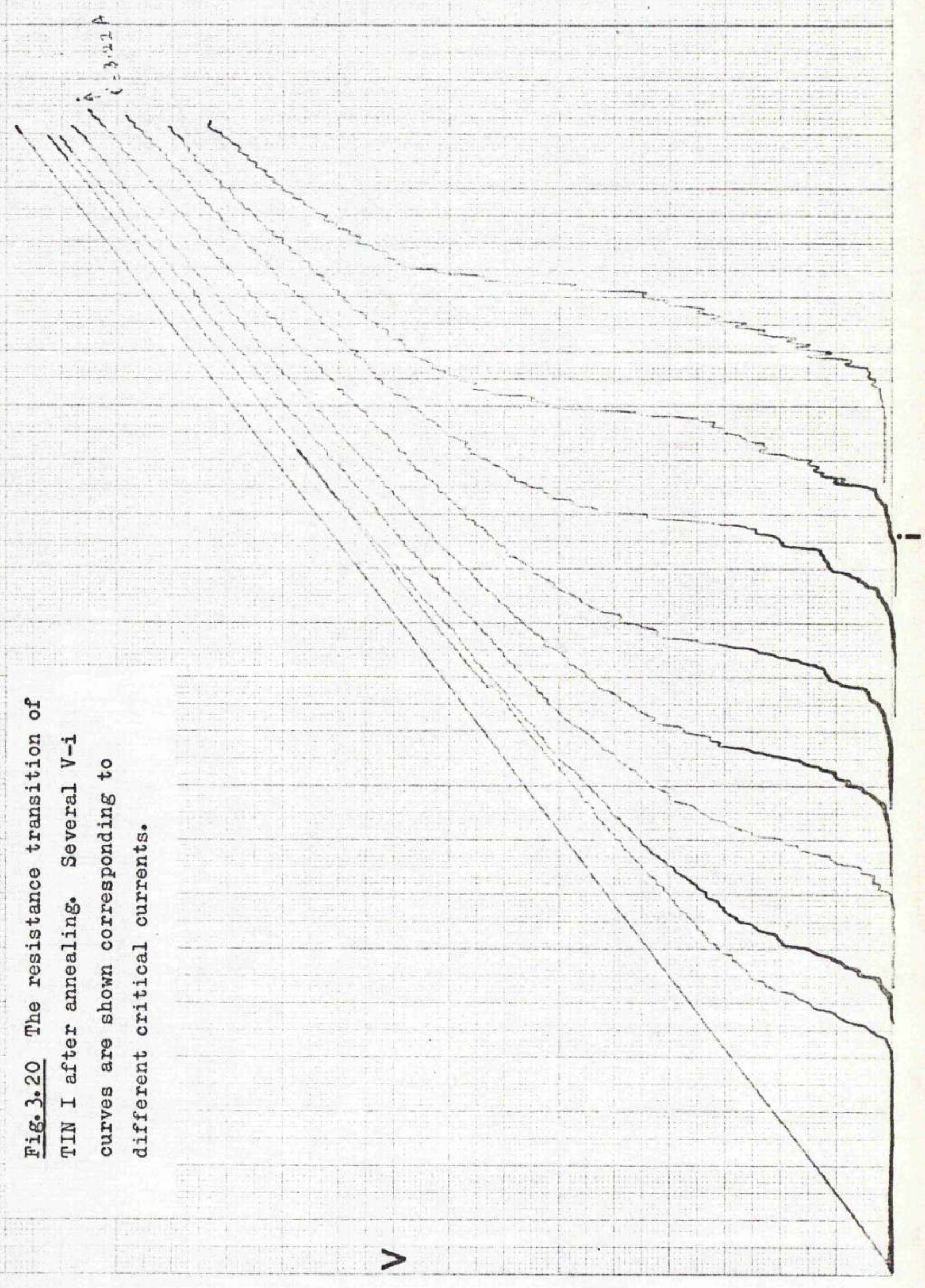




\section{THE SEARCH FOR A SATISFACTORY MODEL}

\section{I DISCUSSION OF THE LONDON MODEL:}

We have seen in the last chapter that the predictions of London's model on the return of resistance do not agree well with experimental results. We shall now investigate if the model is satisfactory from a theoretical viewpoint and whether it can be improved.

London assumed that throughout any normal region of the intermediate state core the magnetic field has the critical value $\mathrm{H}_{\mathrm{c}}$, from which it follows that in such a region (see $\mathrm{d} 2.2$ )

$$
i(r) \propto r \quad \text { and } \quad j(r) \propto \frac{1}{r} \quad \ldots \quad \ldots \quad(4.1)
$$

However he did not show that such a current distribution would result from the structure he suggested, which is shown in Fig.4.1 for the case $i=i_{c^{\circ}}$ Indeed it is easy to see that this structure does not give the required current distribution. Since there is no potential drop across a superconducting region, the interphase boundaries must be equipotential lines and the current lines in the normal regions must intersect them normally. Hence the current lines cannot be parrallel to the axis of the cylindrical wire; thus, in Fig.4.1, $A B C D E$ is a typical current line (noting that, in the first approximation, current only flows along the surface of a superconducting region). Then comparing

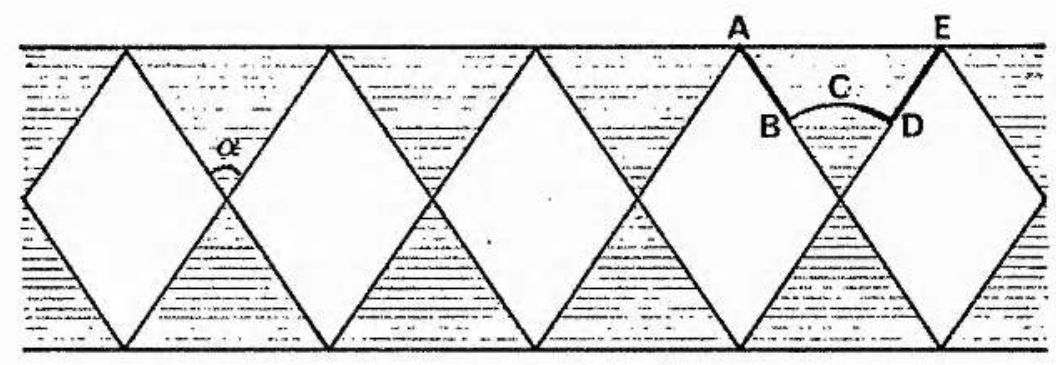

Fig.4.1 Structure proposed by London for the intermediate state in a wire carrying the critical current. The figure has cylindrical symmetry and the shaded areas represent normal regions. 
radii, currents and fields at $B, C$ and $D$ we have:

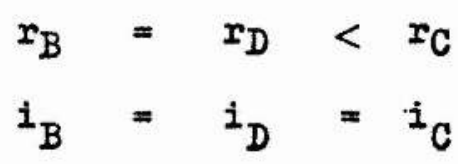

so that

$$
\mathrm{H}_{\mathrm{B}}=\mathrm{H}_{\mathrm{D}}>\mathrm{H}_{\mathrm{C}}
$$

which shows that the field is not constant throughout the normal region.

London did not specify the angle $\alpha$ at the apex of the normal regions. Obviously as $\alpha$ is decreased, the curvature of the current lines will decrease and with it the difference $H_{D}-H_{C^{*}}$ However London's requirement of the field being constant and equal to the critical value throughout the normal region would only be approached if $\alpha$ was made infinitesimally small. However a structure with such a small apex angle is unreasonable on two counts: (a) It would imply a very large number of boundary surfaces per unit length of wire; these would contribute a large surface energy making such a structure energetically unfavourable in Type-I superconductors.

(b) As $\alpha$ approached zero, the shape of the normal regions would approach that of a thin cylindrical disc with a constant current density so that the condition expressed in equation (4.1) would not be satisfied.

To put the dilemma differently: if the interphase boundaries are normal to the axis, then the current density is constant and condition (4.1) is not satisfied; on the other hand if the interphase boundaries are not normal to the axis, then the current lines are curved and the field is not constant throughout the normal region.

Thus no structure would quite satisfy London's criterion of a constant field $\mathrm{H}=\mathrm{H}_{\mathrm{c}}$ throughout the normal regions of a wire in the intermediate state. And as London's derivation of the resistance as a function of current is based. on the assumption that $\mathrm{H}=\mathrm{H}_{\mathrm{C}}$ throughout the normal regions, it is somewhat unrealistic and it is not surprising that London's predictions on the resistance transition differ considerably from experimental results. 


\subsection{AN ALTERNATIVE CRITERION:}

Since London's criterion of $\mathrm{H}=\mathrm{H}_{\mathrm{c}}$ throughout each normal region is unrealistic, we must look for an alternative criterion which must be satisfied by the intermediate state structure. Clearly, for superconducting and normal regions to coexist in quasi-equilibrium conditions, the field along the boundary must be the critical field. Inside any normal region the requirement of $H=H_{\text {. }}$ is not quite that important as a certain amount of supercooling may be allowed for. Both theoretical treatments and experimental results show that considerable supercooling is possible not only in ideal, unflawed specimens, but even in extruded wire. (see for example Deltour et al (1970)).

It therefore seems reasonable to apply the criterion $\mathrm{H}=\mathrm{H}_{\mathrm{c}}$ only to the interphase boundaries; inside any normal region the field may fall slightly. below the critical value, but it must still be greater than the supercooling field and, additionally, the amount of supercooling called for must be minimised so that London's ideal criterion may be approached as far as possible.

The problem, now, is to see whether any structure can be found to satisfy our new criterion and, having found the structure, to evaluate the return of resistance on the basis of the new model. The ultimate test of our alternative criterion will lie in the measure of agreement that can be obtained with experimental results.

\section{3 FINDING THE STRUCTURE:}

To begin with we shall restrict ourselves to the situation when the current is just critical.

When the current through a superconducting wire reaches the critical value, normal regions will nucleate near the surface and such nucleation will occur preferentially around 'weak points' which are regions with a surface energy that is negative due to the presence of impurities or imperfections. Faber (1954, 1955) has shown that each nucleus will first expand to form an annular sheath around the wire and the annulus will then expand both inwards and along 
the length of the wire. Restoration of resistance will occur when the normal regions meet at the axis of the wire and thus break the continuity of the superconducting core. If the ahape of the normal regions is such that $\mathrm{H}_{\mathrm{C}}=\mathrm{H}_{\mathrm{C}}$ on the interphase boundary, then the motion of the boundary will stop and the equilibrium will not be disturbed until the current is further increased. Finding this static equilibrium structure is the first objective.

Shalnikov's (1957) experiments, referred to in 2.3 have shown that the superconducting and normal phases were distributed perpendicularly to the direction of current flow. Also; when the current is critical the field at the surface of the wire is $\mathrm{H}_{\mathrm{C}^{*}}$ These two considerations suggest that the normal regions would be roughly perpendicular to the axis and would be broad near the surface tapering off near the axis. London's picture itself conforms to this rough pattern and can be used as the starting point in our search for a satisfactory boundary shape.

In Fig. 4.2 let ABCDEF represent a typical normal region in a cylindrical wire which has just entered the intermediate state and is in equilibrium with $i=i_{c}$ (the axial width BE has been exaggerated for clarity). ABC and DEF are two interphase boundaries at equilibrium and they must therefore be electric

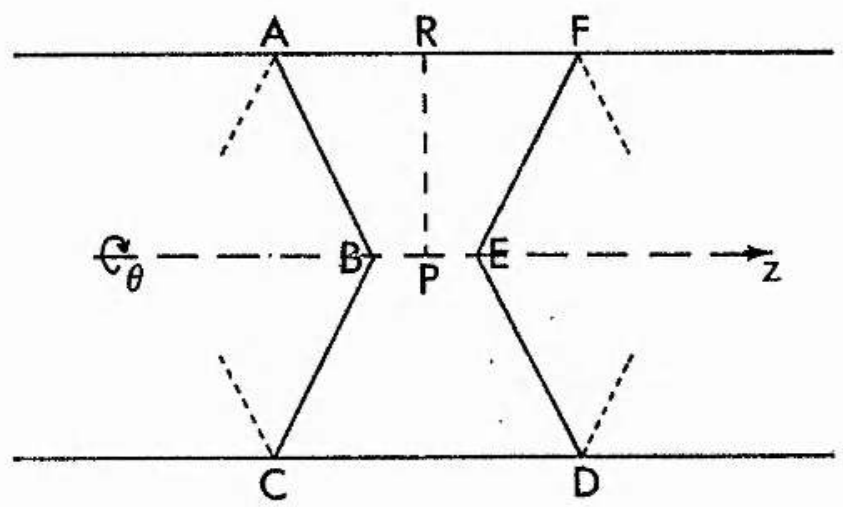

Fig.4.2 Schematic of a typical noxmal region ABCDEF in a wire which has just enetered the intermediate state with $i=i_{c^{*}} \quad$ The axial direction has been chosen as the $z$ axis of a cylindrical coordinate system. 
equipotentials. The potential distribution in the region $A B C D E F$ can be obtained by solving Laplace's equation, in cylindrical coordinates, subject to the following boundary conditions:

(i) The potential is constant on each interphase boundary, i. $\theta$. $\mathrm{V}$ along surface $\mathrm{ABC}=\mathrm{V}_{\mathrm{ABC}}=$ constant $=\mathrm{V}_{\mathrm{A}}$ $\mathrm{V}$ along surface $\mathrm{DEF}=\mathrm{V}_{\mathrm{DEF}}=$ constant $=\mathrm{V}_{\mathrm{F}}$, and

(ii) No current can flow out of the surface of the wire, i.e.

$$
\frac{\partial V}{\partial R}=0 \text { at the surface of the wire. }
$$

Assuming $\mathrm{Ohm}$ 's Law to hold in normal regions, the current distribution can be calculated from the potential distribution using the electromagnetic equations:

$$
\text { and } \left.\quad \begin{array}{l}
\underline{J}=-\sigma \underline{\nabla V} \\
\quad \quad=\int_{S} \underline{J} \cdot d \underline{S}
\end{array}\right\}
$$

which hold for steady flow conditions in a conducting wire (see, for example, Scott (1959) pp. 182-183) and where $\sigma$ represents the normal conductivity of the specimen.

Finally, the magnetic field distribution in the normal region can be obtained using the circuital form of Ampere's Law:

$$
\int \underline{H}(r, z) \cdot d \underline{I}=i(r, z)
$$

noting that, because of symmetry, $H$ and $i$ are independent of the $\theta$ coordinate. In particular we can thus obtain the field distribution along the interphase boundaries. In the present problem we know the kind of field distribution that is required on the boundary surfaces and what we must $f$ ind is the shape of the boundary that will give rise to such a field distribution. The solution of this problem may be attempted in two ways.

(a) Analytically. The problem, as enunciated above, is clearly independent of the $\theta$ direction. Thus the boundary may be represented as a function $F(r, z)$ of $\mathrm{r}$ and $\mathrm{z}$ only. The potential, current and field distributions may be found as a function of $F$ and then the boundary conditions may be applied to find $F(r, z)$. 
(b) Using numerical methods. A guess may be made of the possible boundary shape, arbitrary potential values could be given to the boundaries, and :. Laplace's equation could be solved by using numerical relaxation methods consistent with the boundary conditions on the surfaces of the normal region. This would give the potential distribution and using this the current and field distributions could be evaluated numerically. The knowledge of the field distribution along the interphase boundary would enable a better guess of the boundary shape to be made and the process repeated as many times as necedsary until a boundary shape is obtained that satisfies the requirement of constant field along the boundary. This method of solution is only made possible by the availability of computers.

In this work we have used numerical methods to find satisfactory boundaries. This method had the advantage of producing actual numbers for the potential, current and field at points on a regular array in the normal region and of thus giving a real feel of what was happening. In addition this approach served as an introdu ction to computational techniques.

An attempt at solving the problem by analytical methods has since been made by Mocill (1968) who found that the ultimate solution of a system of equations still required the use of approximation methods. Thus it would appear that the analytical method had no advantages over the numerical approach which has been followed in this work.

4.4 THE RELAXATIONAL SOLUTION OF LAPLACE'S EQUATION IN CYLINDRICAL COORDINATES: The relaxational solution to a differential equation consists of values of the wanted function, say $w$, at a number of equally spaced points of subdivision along the range of integration. Thus, if the range of integration is given by $x=a$ to $x=b$ and if the range be divided into $n$ intervals of length $h$ each, the relaxation process will yield values of $w$ at the points $x=a+h, a+2 h$, $\ldots \ldots a+(n-1) h$, the values at $x=a$ and $x=b$ having been specified by the boundary conditions. 
In applying a relaxation method, we shall need to replace derivatives by certain finite difference approximations and we shall now derive these.

In Fig. 4.3 let 0 represent a typical point of subdivision in the range of integration along, say, the $x$ axis and let 1 and 3 represent adjacent points of sub-

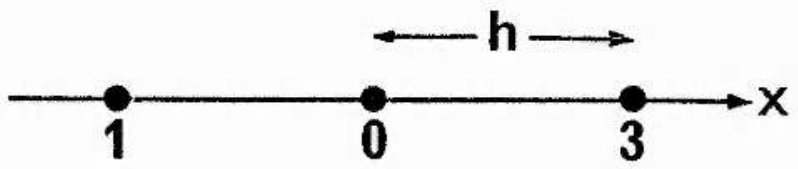

Fig.4.3 Three typical points along the range of integration.

division. Using Taylor's series

the function $w$ can be expanded in the neighbourhood of 0 :

$$
w=w_{0}+\left(\frac{d w}{d x}\right)_{0}\left(x-x_{0}\right)+\frac{1}{2 !}\left(\frac{d^{2} w}{d x^{2}}\right)_{0}\left(x-x_{0}\right)^{2}+\frac{1}{3 !}\left(\frac{d^{3} w}{d x^{3}}\right)_{0}\left(x-x_{0}\right)^{3}+\ldots .
$$

Replacing $x$ successively by $x_{0}+h$ and $x_{0}-h$ we get, in turn,

$$
\begin{aligned}
& w_{1}=w_{0}+h\left(\frac{d w}{d x}\right)_{0}+\frac{h^{2}}{2}\left(\frac{d^{2} w}{d x^{2}} b_{0}+\frac{h^{3}}{6}\left(\frac{d^{3} w}{d x^{3}}\right)_{0}+\frac{h^{4}}{24}\left(\frac{d^{4} w}{d x^{4}}\right)_{0}+\ldots\right. \\
& w_{3}=w_{0}-h\left(\frac{d w}{d x}\right)_{0}+\frac{h^{2}}{2}\left(\frac{d^{2} w}{d x^{2}}\right)_{0}-\frac{h^{3}}{6}\left(\frac{d^{3} w}{d x^{3}}\right)_{0}+\frac{h^{4}}{24}\left(\frac{d^{4} w}{d x^{4}}\right)_{0}-\ldots
\end{aligned}
$$

so that

$$
w_{1}+w_{3}=2 w_{0}+h^{2}\left(\frac{d^{2} w}{d x^{2}}\right)_{0}+o\left(h^{4}\right)
$$

where $O\left(h^{4}\right)$ contains terms with fourth or higher powers of $h$. Assuming $h$ to be small, $O\left(h^{4}\right)$ may be neglected and we have

$$
h^{2}\left(\frac{d^{2} w}{d x^{2}}\right)_{0}=w_{1}+w_{3}-2 w_{0}
$$

which is the finite difference approximation to $\left(\frac{d^{2} w}{d x^{2}}\right)_{0}$

Similarly we have

$$
w_{1}-w_{3}=2 h\left(\frac{d w}{d x}\right)_{0}+o\left(h^{3}\right)
$$

and neglecting terms with $h^{3}$ or higher powers of $h$, we obtain

$$
h\left(\frac{d w}{d x}\right)_{0}=\frac{1}{2}\left(w_{1}-w_{3}\right)
$$

The problem we are interested in here has cylindrical symmetry. It is therefore appropriate to work in cylindrical polar coordinates. In these coordinates, Laplace's equation for a potential function $V$ is:

$$
\frac{\partial^{2} V}{\partial r^{2}}+\frac{1 \partial V}{r}+\frac{\partial^{2} Y}{\partial z^{2}}=0
$$


when $V$ is independent of $\theta$ as in our problem.

The entire range of integration (in our case, the normal region) is divided into a square net of side $h$, the two main coordinates being the $r$ and $z$ coordinates. We note that since $V$ is independent of $\theta$, the problem is really a quasi-twodimensional one, except that the appropriate Laplace's equation is the one for the cylindrical case. Fig. 4.4 shows a portion of the square mesh; let 0 be a typical node and let its nearest neighbours be termed $1,2,3$ and 4 as indicated

3

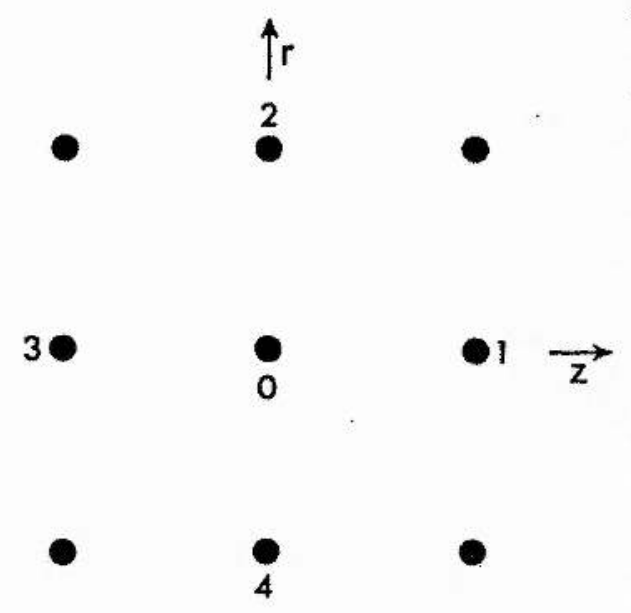

Fig. 4.4 Part of the square mesh used in the numerical solution of Laplace's equation.

in the figure. The finite difference approximations (4.2) and (4.3) applied to node 0 give:

$$
\begin{aligned}
\frac{\partial^{2} V}{\partial r^{2}} & =\frac{V_{2}+V_{4}-2 V_{0}}{h^{2}} \\
\frac{\partial^{2} V}{\partial z^{2}} & =\frac{V_{1}+V_{3}-2 V_{0}}{h^{2}} \\
\frac{1}{x} \frac{\partial V}{\partial r} & =\frac{1}{r_{0}} \frac{V_{2}-V_{4}}{2 h}
\end{aligned}
$$

so that Laplace's equation in cylindrical coordinates (4.4) becomes

$$
4 v_{0}=v_{1}+v_{3}+\left(1+\frac{h}{2 r_{0}}\right) v_{2}+\left(1-\frac{h}{2 r_{0}}\right) v_{4} \quad \ldots
$$

This is the important iterative equation which enables the value of $V$ at node 0 to be determined from the values of $V$ at points $1,2,3$ and 4.

Nodes on the axis $(r=0)$ constitute a special case as the term in $\frac{1}{r}$ in equation (4.4) becomes indeterminate and must be replaced by

$$
\operatorname{Lim}_{x \rightarrow 0} \frac{1}{x}\left(\frac{\partial V}{\partial x}\right)=\frac{\partial^{2} V}{\partial x^{2}}
$$




$$
2 \frac{\partial^{2} v}{\partial r^{2}}+\frac{\partial^{2} v}{\partial z^{2}}=0 \quad \cdots \quad \cdots
$$

We also note that when the node of interest 0 is on the axis, the axial symmetry of the problem implies that $v_{2}=v_{4^{\circ}}$. Now using $(4.2)$ and $(4.3)$, (4.6) gives

$$
6 \mathrm{~V}_{0}=\mathrm{V}_{1}+4 \mathrm{~V}_{2}+\mathrm{v}_{3}
$$

which is the iterative equation applicable to nodes on the axis.

\subsection{APPLICATION TO THE PREISENT PROBLIM:}

Our problem can be considerably simplified by taking into consideration the various symmetries involved. (See Fig. 4.2, page 4.4)

(a) There is cylindrical symmetry, so that the potential $\mathrm{V}$ is independent of the $\theta$ direction. The problem can therefore be treated as a two-dimensional one, say in the plane of Fig.4.2, with the proviso that the appropriate form of of Laplace's equation to use is that which is expressed in cylindrical coordinates.

(b) The axis of the cylinder, i.e. the $z$ axis, is an axis of symnetry. The solution of Laplace's equation may therefore be confined the region ABEF. (c) However the region ABEF is itself symmetric around the line PR. We may therefore further confine the solution of the problem to the region ABPR. As $A B$ and $E F$ are interphase boundaries, they must be equipotential lines and the symmetry just mentioned then implies that PR must also be an equipotential line.

The problem has thus been reduced to solving Laplace's equation in the region $A B P R$, with the following boundary conditions:

(i) $\mathrm{AB}$ and $\mathrm{PR}$ are equipotentials.

(ii) AR being on the surface of the wire, no current can pass across it, i.e. $\left(\frac{\partial V}{\partial x}\right)=0$ along $A R$.

(iii) $\mathrm{PB}$ is really the axis of the cylinder so that the relaxation equation (4.7) must be used for nodes on $\mathrm{PB}$.

Fig. 4.5 shows how a region such as ABPR can be covered by a square mesh of nodes to obtain a relaxational solution.

A rectangular coordinate system 


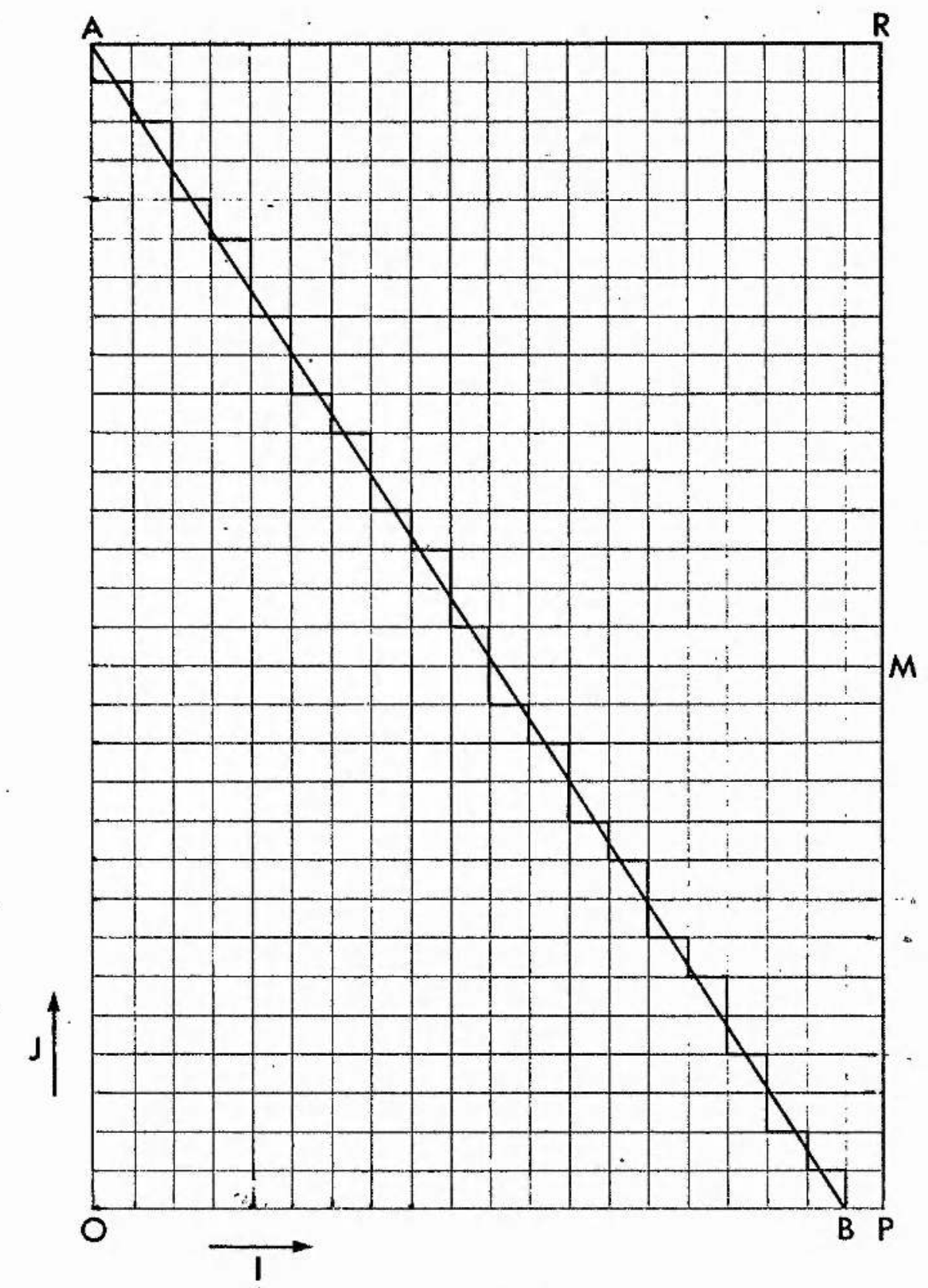

Fig.4.5. A typical region ABPR (see Fig.4.2) covered by a square mesh for numerical solution of Laplace's equation. The boundary $A B$ is approximated by a zigzag of mesh lines as shown in the figure. The line op represents the axis of the wire.

holps to identify the mesh points and the origin of the coordinate system is taken at 0 , the projection of $A$ on the axis of the wire; this choice helps to make the computer programme a little more flexible. The axis of the wire constitutes the $I$ axis and the $J$ axis is then the direction of the radius $r$. For computational convenience the origin is labelled $(1,1)$ instead of $(0,0)$ as is usual in coordinate systems. Points along AR have the maximum $J$ 
coordinate and we shall designate this value by JMAX. Similarly points along RP have the maximum I coordinate IMAX. Any arbitrary boundary shape AB is approximated by a zigzag of mesh lines and Fig. 4.5 shows an example of this; making the mesh finer improves the approximation.

The main object of the computer calculations is to find the distribution of magnetic field along any specified interphase boundary. When a boundary satisfying our criterion of field constancy has been found, we would wish to know

(a) the magnetic field distribution throughout the normal region - this would give us an idea of the supercooling involved, and

(b) the ratio of the electrical resistance of such a region to that of a similar region (AOPR) in a fully normal wire.

In practice it is easier to write one programme to achieve all this. The amount of computer time required to carry out the steps required for (a) and (b) is negligible compared with the time required to achieve a numerical solution of Laplace's equation.

The computer calculations involve the following steps:

I. Any boundary of interest is approximated by a zigzag of mesh lines and the coordinates of the nodes on this zigzag line are fed into the computer.

II. Boundary conditions. $A B$ and $P R$ are equipotentials. Without any loss of generality they are allotted the arbitrary potential values 100.0 and 0.0 respectively. The other boundary condition is that $\left(\frac{\partial V}{\partial r}\right)=0$ along the surface of the wire. This is achieved by setting $V(I, J M A X)=V(I, J M A X-I)$ where $V(I, J)$ represents the potgential at any point of coordinates I and J. III. Solution of Laplace's equation. Using equations (4.5) and (4.7), and subject to the above boundary conditions, the computer evaluates $V(I, J)$ sweeping through all values of $(I, J)$. As the number of iterative sweeps increases, the values of $V(I, J)$ begin to approach the exact values which are a solution to Iaplace's equation. For our purposes it was sufficient to 
find the value of $V$ to an accuracy of $0.1 \%$. By comparing the numerical solution of Laplace's equation in the case of a fully normal cylinder with the known analytic solution it was found that an accuracy of about $0.1 \%$ was being achieved when successive computer sweeps did not cause any $V(I, J)$ to change by more than 0.001. The computer was accordingly. instructed to stop iterating when this atage is reached. Thus for every $(I, J)$ we obtain the value of $V$ at that point which is a solution of Laplace's equation; in other words we obtain the potential distribution throughout the normal region.

IV. The current and field distribution. In order to find the value of the magnetic field at any point $M\left(I_{M}, J_{M}\right)$, it is necessary to know the total current $i_{M}$ flowing within a cylindrical core of the same radius as $M$. Then we can use the circuital form of Ampere's law:

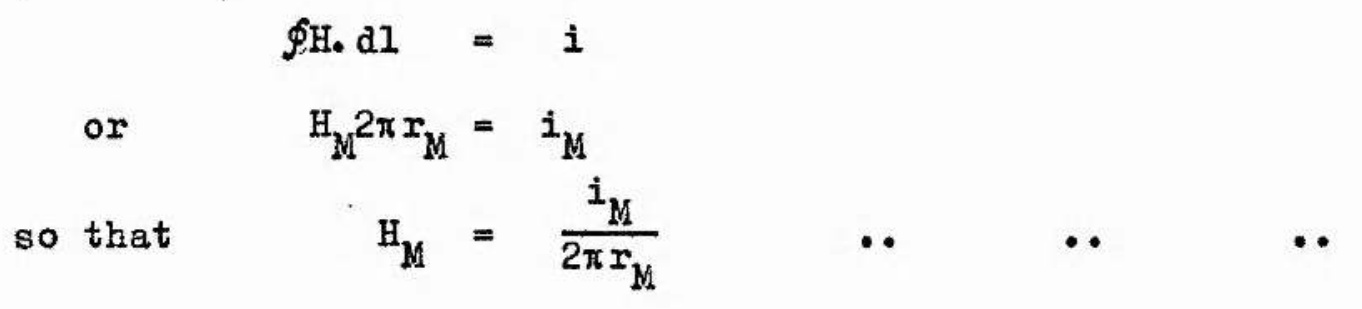

The total current $i_{M}$ can be found by adding the currents through all the volume elements defined by the mesh on any one cross-sectional plane, e. g. that through PR. This may be best understood by considering a particular example. In Fig.4.6(a) XY represents the axis of the:wire and A, B, ... N are mesh points with coordinates $A\left(I_{A}, J_{A}\right)$ etc.. In this case we note that $X X$ is also the I axis. Assuming $\mathrm{Ohm}$ 's Law to hold in the normal region, the average current density in the element ACDB may be approximated by:

$$
j_{e l}=\left\{\frac{v_{C}+v_{A}}{2}-\frac{v_{D}+v_{B}}{2}\right\} \sigma
$$

where $\sigma$ is the normal state conductivity. This current density is now flowing through a little cylinder of length $A B$ and radius $A C$, whose crossection is

$$
B_{e 1}=\pi \cdot A^{2} \cdot \cdots=\pi \cdot 1^{2} \cdot=\pi
$$

Hence the total current through the element ABCD is

$$
i_{e l}=j_{e I} \times \varepsilon_{e l}=\left\{\frac{v_{C}+v_{A}}{2}-\frac{v_{D}+V_{B}}{2}\right\} \sigma \pi
$$




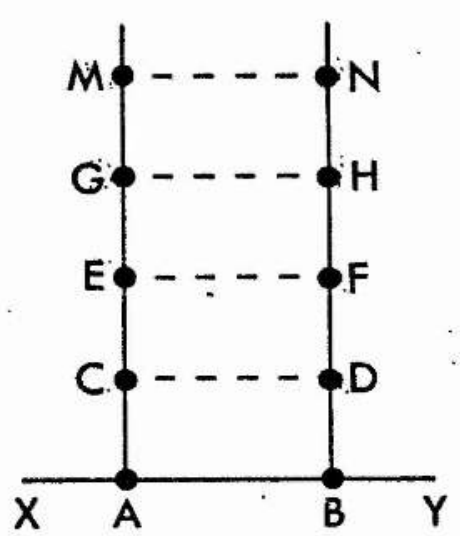

(a)

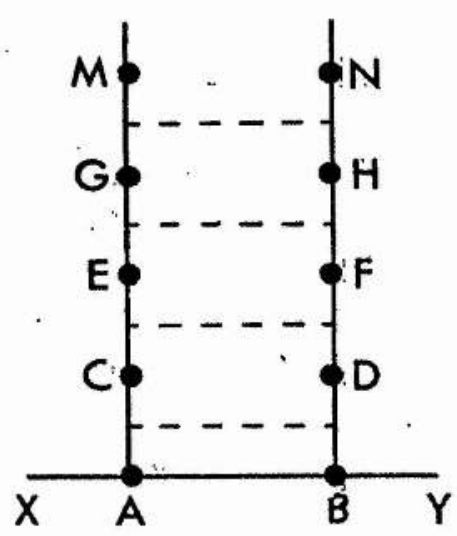

(b)

Fig.4.6 Two vays of dividing the computational mesh into volume elements for the purpose of calculating the current and magnetic field at mesh points.

This is now the total current flowing through a loop of radius AC. Hence the magnetic field at $\mathrm{C}$ is given by

$$
H_{C}=\frac{i_{C}}{2 \pi(A C)}=\frac{i_{e l}}{2 \pi .1}=\frac{\sigma}{2}\left\{\frac{V_{C}+V_{A}}{2}-\frac{V_{D}+V_{B}}{2}\right\}
$$

Since the factor $\frac{\sigma}{2}$ is constant and will appear in every single element, it may be taken out of the actual numerical evaluation and the computer only needs to calculate

$$
\mathrm{H}_{\mathrm{C}} \sim\left\{\frac{\mathrm{V}_{\mathrm{C}}+\mathrm{V}_{\mathrm{A}}}{2}-\frac{\mathrm{V}_{\mathrm{D}}+\mathrm{V}_{\mathrm{B}}}{2}\right\}
$$

Similarly we next consider the element CEFD. The average current density through this element is

$$
j_{e l}=\left\{\frac{V_{E}+V_{C}}{2}-\frac{V_{F}+V_{D}}{2}\right\} \sigma
$$

The volume element involved is now the cylindrical sheath of inside radius AC and outside radius AE. The crossectional axea of this sheath is

$$
s_{e l}=\pi\left(A E^{2}-A C^{2}\right)=\pi\left(2^{2}-I^{2}\right)=3 \pi .
$$

so that the current through this element is

$$
i_{e l}=3 \pi \sigma\left\{\frac{V_{E}+V_{C}}{2}-\frac{V_{F}+V_{D}}{2}\right\}
$$

The total current passing through the loop of radius AE, say $i_{E}$, is the sum of the current through the element CEFD and the current $i_{C}$ through the element 
$A C D B$ :

$$
i_{E}=i_{e l}+i_{C}=3 \pi \sigma\left\{\frac{V_{E}+V_{C}}{2}-\frac{V_{F}+V_{D}}{2}\right\}+i_{C}
$$

where $i_{C}$ has already been determined. The magnetic field at is now

$$
\mathrm{H}_{\mathrm{B}}=\frac{i_{\mathrm{E}}}{2 \pi \cdot \mathrm{AE}}
$$

Similarly the calculations may be continued to find $\mathrm{H}_{G}, \mathrm{H}_{M}$ etc..

The above approximation is not the only method by which the current can be calculated. Other approximations are possible - for example, the current and volume elements may be chosen as shown in Fig.4.6(b) instead of as show in Fig.4.6(a). However although various forms of approximation are possible, they all give values of $H(I, J)$ which are within $0.5 \%$ of each other in the case of the finest mesh used, and this is an indication of the accuracy. of the calculations.

V. The resistance ratio. The resistance ratio was determined as follows (see Fig.4.7). For any particular interphase boundary $A B$, the total current $I_{I}$ passing across $P R$ (i.e. the diametral section of the wire at $P$ ) is found as indicated above. This is compared with the total current $I_{N}$ which would have passed through $P R$ if the whole wire had been normal, i.e. if the boundary was simply $A O$ and was given the

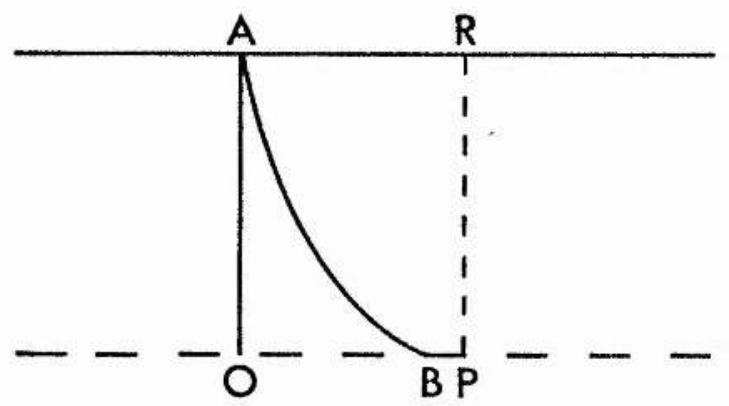

Fig.4.7 Sketch illustrating how the resistance ratio was determined for a typical interphase boundary $A B$.

The resistance ratio is then given by:

$$
\frac{R_{\text {Intermediate }}}{R_{\text {Normal }}}=\frac{I_{N}}{I_{I}}
$$

for

$$
V=I_{N} R_{N}=I_{I} R_{I}
$$

All the calculations detailed above were carried out by computers. The 
process consisted of 'guessing' a boundary and feeding it to the computer, then using the field variation along that boundary to try and improve the boundary so as to get a better constancy of magnetic field, and feeding the new boundary to the computer. And so on, until a shape of boundary was found which would satisfy our criterion of $\mathrm{H}$ constant along the boundary. The whole process was slow because it was difficult to 'adjust' a boundary so as to make it satisfy our criterion better.

Initially the work was carried out on an IBM 1620 computer available locally. By modern standards this is a slow computer and later, for finer meshes the much faster ATLAS and IBM $360 / 44$ computers were used. In the finest mesh used the radius of the wire corresponded to 200 mesh divisions. The computer programmes were written in FORTRAN language and a typical example is given in Appendix A. 


\section{THE NEW MODEL}

5. 1 THE NEW MODEL $\left(1=i_{c}\right)$

In the previous chapter we have suggested a realistic criterion which must be satisfied by the intermediate state structure and we have established the methods of looking for such a structure. Using these methods we find that there is no unique solution to the problem but that a structure to satisfy the criterion of $\mathrm{H}=\mathrm{H}_{\mathrm{C}}$ on the interphase boundaries can be found for any given value of the structure periodic length $2 d$ upto a minimum value $\mathrm{d}_{\min }=0.715 \mathrm{a}$ where $\mathrm{a}$ is the radius of the wire. In Fig.5.1 three such structures are show in axial crossection. The axial width $w$ of the normal regions at the centre of the wire falls rapidly to zero as $d$ approaches

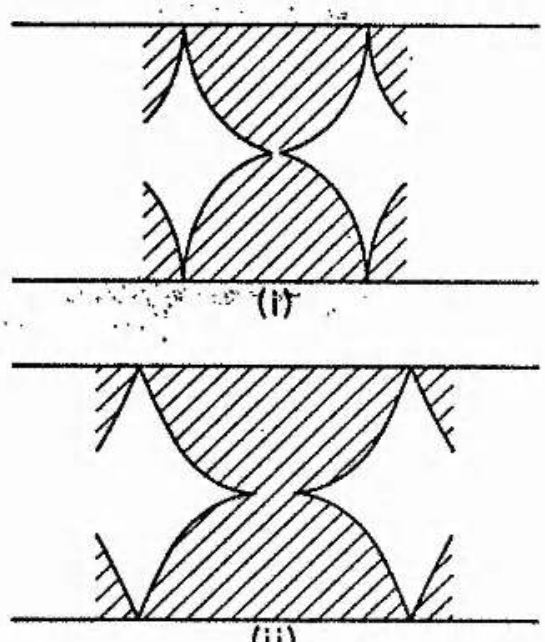
(ii)

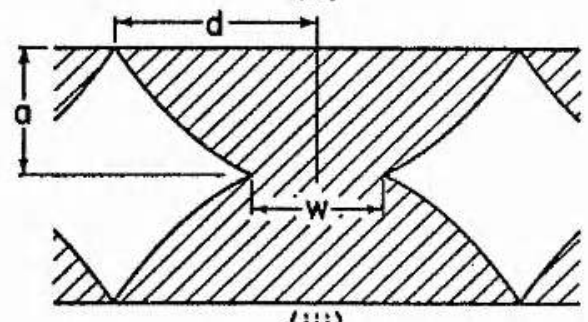
(iii)

$0.715 a$ and we have found it impossible to find satisfactory structures for d smaller than $0.715 \mathrm{a}$. In Fig. 5.1 the axial width in case (i) has been exaggerated to make its existence Fig. 5.1 Equilibrium intermediate state structures show in axial cross-section for three different values of periodicity: (i) $d=$ $0.715 a$, (ii) $d=a$, (iii) $d=$ 1. 5 a. clear - actually in this case $w=a / 140$.

It is not entirely surprising that there should be a minimum periodic length below which no structure can be found to satisfy our criterion. For, in the limit of very small $d$ the current density in the normal region would approach constancy rather than satiofy the requirement of $j(r) \propto 1 / r$. It is also intuitively satisfactory to find that an abrupt increase in the resistance 
should correlate with a minimum value of d.

It may be pointed out that for any given value of d we required about fifteen tries in order to obtain a boundary that gave a constant field along the boundary. In each case the work was first carried out on a coarse mesh and then refined on a fine mesh until constancy of the field at the boundary could be achieved to within about 1 percent.

As we have pointed out in $\mathbf{1} 4.1$, no structure quite satisfies the ideal requirement of $\mathrm{H}=\mathrm{H}_{\mathrm{c}}$ throughout the normal regions. For structures which have $\mathrm{H}=\mathrm{H}_{\mathrm{C}}$ on the boundary the field in the normal regions is less than or equal to $\mathrm{H}_{\mathrm{c}}$. There is thus a certain amount of supercooling and, in choosing the optimum structure (i.e. the optimum value of d) from the solutions we have obtained, we must minimise the supercooling required per unit length of wire. Quantitatively this amounts to minimising

$$
\theta=\frac{1}{H_{c}{ }^{2} \mathrm{~d}}\left\{\frac{\mathrm{H}_{\mathrm{c}}{ }^{2}}{8 \pi} \mathrm{V}_{\mathrm{n}}-\int_{\mathrm{V}_{\mathrm{n}}} \frac{\mathrm{H}^{2}}{8 \pi} \mathrm{dV}\right\}
$$

where $V_{n}$ is the volume of the normal region. Values of $e$ have been calculated and Table 5.1 shows the variation of $\theta$ with $\frac{d}{a}$. As ds only to be expected e decreases as $\frac{\mathrm{d}}{\mathrm{a}}$ becomes smaller, the supercooling involved is reduced as the structure becomes thinner.

We knov that for Type-I superconductors the normal-superconducting interfaces have a positive surface energy. Hence, in choosing the optimum structure we must also minimise the surface energy s per unit length of wire. We have calculated values of $s$ for our structures in the case of Indium which is a strongly liype-I superconductor $\left(\Delta \sim 3.4 \times 10^{-5} \mathrm{~cm}.\right)$ and these are given in Table 5.1 for three different radii. It can be seen that for values of radii normally used in experimental work, $\mathbf{s}$ is negligible in comparision with $\theta$.

It follows that in choosing the optimum structure, it is e that is the determining factor, and since $\theta$ is minimum for the structure corresponding to $d_{\text {min }}$, we suggest that at $i=i_{c}$ the intermediate state structure in the wire 


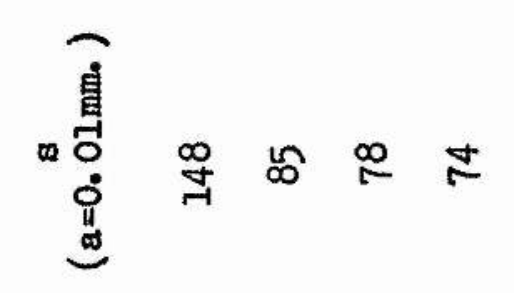<smiles>C#CC#CC#CC#N</smiles>

$$
\text { वा क }
$$




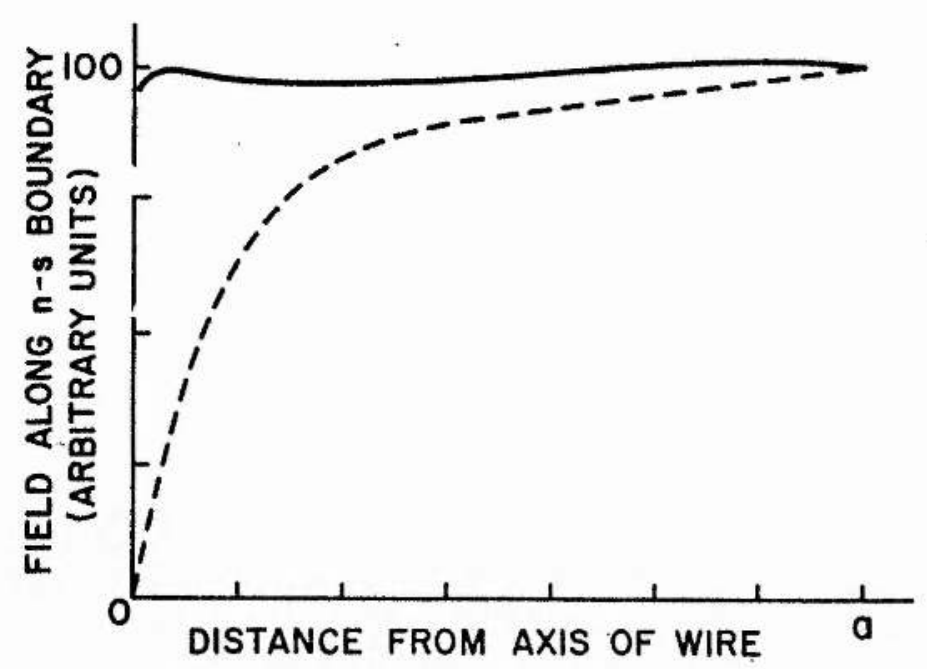

Fig. 5.2 Variation of magnetic field H along interphase boundary. Solid line, this model; broken line, London's model (assuming $d=0.715 a$ )

is that shown in Fig.5.1(i). The variation of magnetic field along any interphase boundary in this structure is compared with the equivalent variation in London's model in Fig.5.2. If one accepts the criterion that the magnetic field along a normal-superconducting boundary should be $H_{c}$, it is clear that the present model is much more satisfactory than London's model.

The normalised resistance of the structure shown in Fig.5.1(i) is

$$
\rho=\left(\frac{R}{R_{n}}\right)_{i=i_{c}}=0.69
$$

which agrees much better with experimental values than London's figure of 0.5

The above value of $\rho$ is calculated on the assumption of a perfectly periodic structure. We note however that $\theta$ is only a slowly varying function of $(a / a)$ and in the presence of any sharp local variation of surface energy, caused by matallurgical defects or impurities, it is possible that the optimum value of $d$ will be slightly larger than 0.715 a and the probable local structure will be that corresponding to doptimum. The values of $\rho$ corresponding to the equilibrium structures for various values of $(a / a)$ are shown in Table 5.2 and it can be seen that any distortion in the periodicity will, if anything, cause an increase in the value of $p$ for the whole wire. 


\section{TABLE 5.2}

$\frac{\mathrm{d}}{\mathrm{a}}$

$$
\rho
$$

0.715

0.69

1.00

0.724

1.24

0.747

1.53

0.788

5.2 THE SUPERCOOLING INVOLVED IN THE STRUCTURE:

As indicated earlier the magnetic field does not quite have the critical value throughout the normal regions. Overall, the amount of supercooling may be characterised by the summing $\mathrm{H} / \mathrm{H}_{\mathrm{c}}$ over the whole of any normal region, $\mathrm{H}$ being the actual magnetic field at any point. For the structure of Fig.5.I(i)

$$
\mathrm{S}=\Sigma \frac{\mathrm{H}}{\mathrm{H}_{\mathrm{c}}} \text { over a normal region }=0.82
$$

The actual distribution of field over the normal region can be understood by referring to Fig. 5.3 where the essential part of the structure is shown at
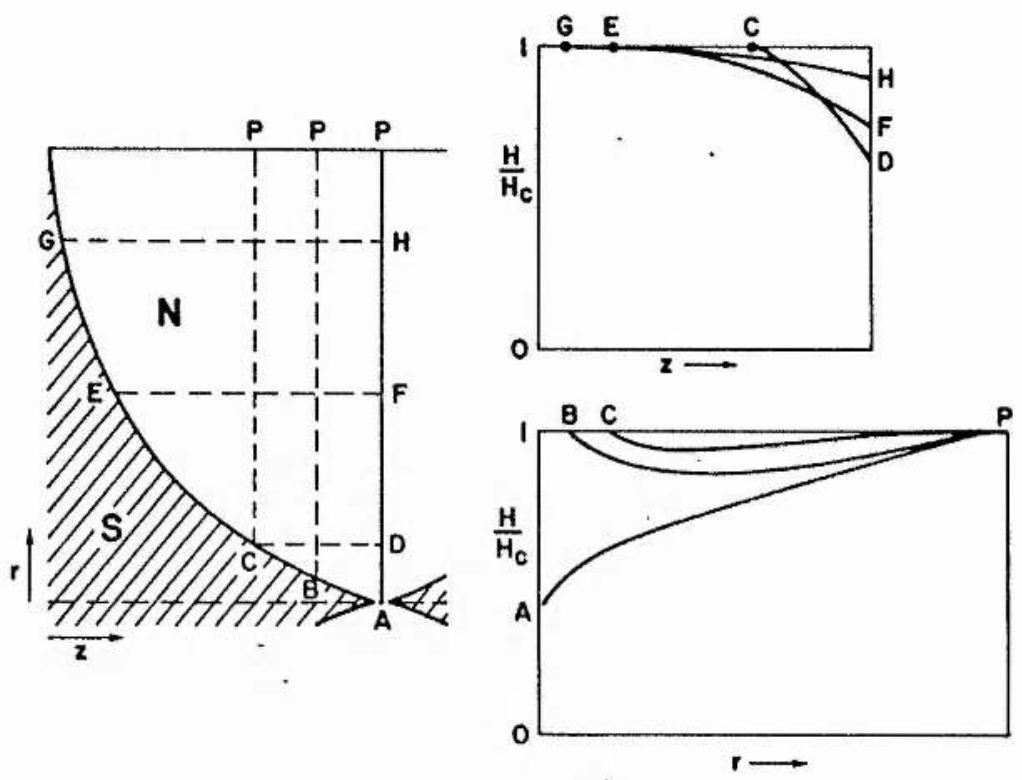

Fig. 5.3 Distribution of the magnetic field in a normal region: A quarter of the axial section of a normal region is shown at left, and the field distribution along the lines $A P, B P, C P$ and $C D, E F$ and $G H$ is shown at right. 
left and the field distribution along a few interesting directions is shown at right. It can be seen that apart from a small region around $A D$ the field is everywhere greater than $0.8 \mathrm{H}_{\mathrm{c}^{*}}$ At $\mathrm{A}$ the value of $\mathrm{H}$ decreases to about $0.5 \mathrm{H}_{\mathrm{c}^{\circ}}$

We note that for computational purposes the axial width $w$ of a normal region cannot be smaller than one mesh unit length. In practice w should be infinitesimally small but finite, so that the values of $\mathrm{H} / \mathrm{H}_{\mathrm{c}}$ in this region will really be considerably higher than shown in Fig.5.3. The effect of this correction will become smaller as one moves away from A.

We have seen that in an ideal solution to the problem the current density in the normal regions should be inversely proportional to $x$. Although our solution does not quite satisfy this ideal condition, it is clear from the shape of the boundary that the current density will be highest at A and will decrease progressively as the distance from the axis increases. It follows that joule heating will be relatively greater near the axis. On the other hand, in any actual experiment the wire is cooled from the surface inward. Thus the axial region is likely to have a slightly higher temperature than the surface. This is an additional reason why the supercooling near A will really be smaller than shown in Fig.5.3.

We note that experiments on resistance transitions in superconducting wires are all carried out at temperatures close to the critical temperatures. Ginzburg (1956) has show that under these conditions the lower limit of magnetic field $\mathrm{H}_{1}$ to which an ideal Type-I superconductor can be supercooled is given by

$$
S_{1}=\frac{H_{1}}{H_{c}}=\sqrt{2} k
$$

For $T$ close to $T_{c}$, Faber (1957) experimentally obtained the following values of $S_{1}$ : Tin: $S_{1}=0.164$ and Indium: $S_{1}=0.112$. Using values of $k$ deduced from experimental measurements of the penetration depths, these results agree well with Ginzburg's theoretical formula. Faber's measurements were carried out on wires about $1.5 \mathrm{~mm}$. in diameter which is of the same order as that of 
wires used in resistance transition experiments. Besides, recent experiments by Deltour et al (1970) with extruded polycrystalline Indium wires, similar to those used in our experiments give $s_{I} \sim 0.36$.

The maximum supercooling involved in the present model is considerably less than that allowable on the basis of Ginzburg's theory and the experiments of Faber and Deltour et al. Thus the structure we have proposed for the intermediate state when $i=i_{c}(F i g .5 .1(i))$ satisfies the thermodynamic equilibrium condition that $\mathrm{H}=\mathrm{H}_{\mathrm{c}}$ along the boundary while the supercooling required is well within acceptable limits.

5.3 THE NEW MODEL $\left(i_{c}<i\right)$ :

When the current through the wire rises above $i_{c}$, it may be assumed that the superconducting cores will shrink to a new equilibrium shape for each value of current, so as to maintain the condition $\mathrm{H}=\mathrm{H}_{\mathrm{c}}$ along the boundaries. Obviously the magnetic field in the continuous normal sheath along the surface of the wire will be greater than $\mathrm{H}_{c^{*}}$ Using the same computational techniques as before, boundaries to satisfy the criterion have been found for several values of $i>i_{c}$, and two of them are indicated by the broken Iines in Fig.5.4.

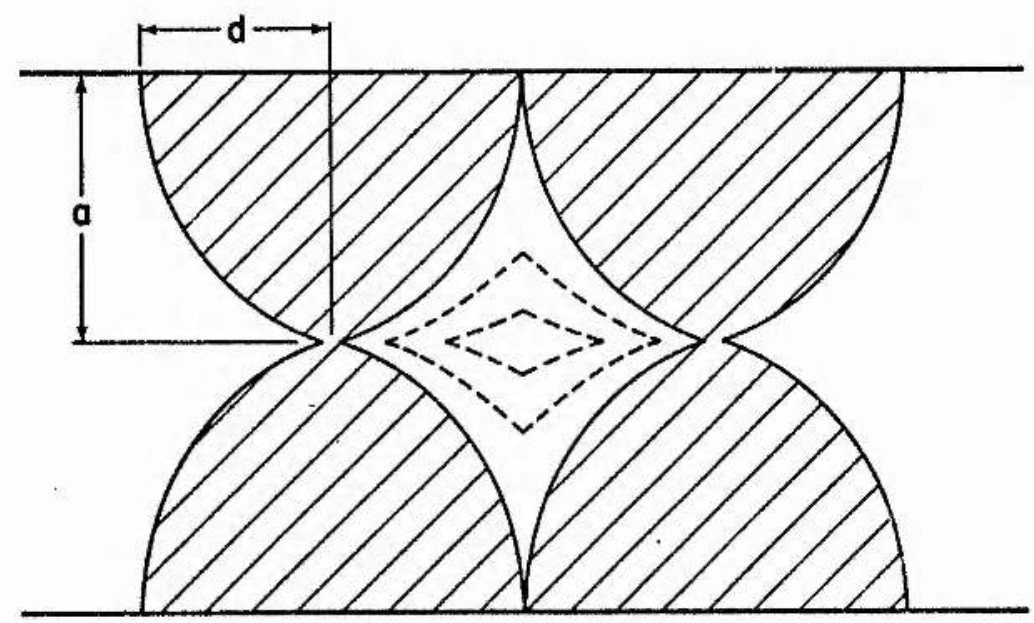

Fig.5.4 Possible evolution of the intermediate state structure as $i$ increases over $i_{c}$. The solid lines show the interphase boundaries at $i=i_{c}$. As $i$ increases the superconducting cores may shrink to successive equilibrium boundaries; two such boundaries are shown in dotted Iines. 
It can be seen from the figure that as the current increases, so the axial width of the normal regions increases (the periodicity stays the same), and it follows that the supercooling in the normal regions increases. In particular the magnetic field at the centre of the axial width becomes very small and at $i=2.25 i_{c}$ this is less than $0.1 H_{c^{\circ}}$ This amount of supercooling cannot be justified and such a model is therefore unacceptable even though it gives a return of resistance curve in reasonable agreement with experimental results.

An alternative possibility, first suggested by London in his model, is that for $i>i_{c}$ there will be an intermediate state core and a normal sheath in the wire. As the current increases the core will shrink in diameter and the normal sheath will expand inwards until finally the whole wire becomes normal. The core will consist of normal and superconducting regions and, once again, the structure must satisfy the criterion that $\mathrm{H}=\mathrm{H}_{\mathrm{C}}$ on the interphase boundaries. We therefore suggest that the interphase boundaries in the intermediate state core will be identical in shape to the boundaries proposed for the case of $i=i_{c}$. The periodicity of the structure will therefore be a function of the radius of the internediate state core. Fig.5.5 (page 5.9) shows the structure for three different values of current and it can be seen that as the current increases so the periodicity of the intermediate state structure decreases. The magnetic field distribution in the normal regions of the intermediate state core is now the same as discussed in 85.2 and the supercooling involved is therefore within acceptable limits.

\subsection{COMPARISION WITH EXPERINENT. THE RESISTANCE TRANSITION:}

Resistance valueg for structures corresponding to various values of current have been numerically evaluated and they have been used to plot a smooth curve in Fig. 5.6 where a few of our experimental points have been show for comparision. In Table 5.3 the resistance values predicted by oux model have been compared with experimental values obtained by us and other workers on 'thick' wires near $T_{c}$ (we shall see later that in thin wires certain 


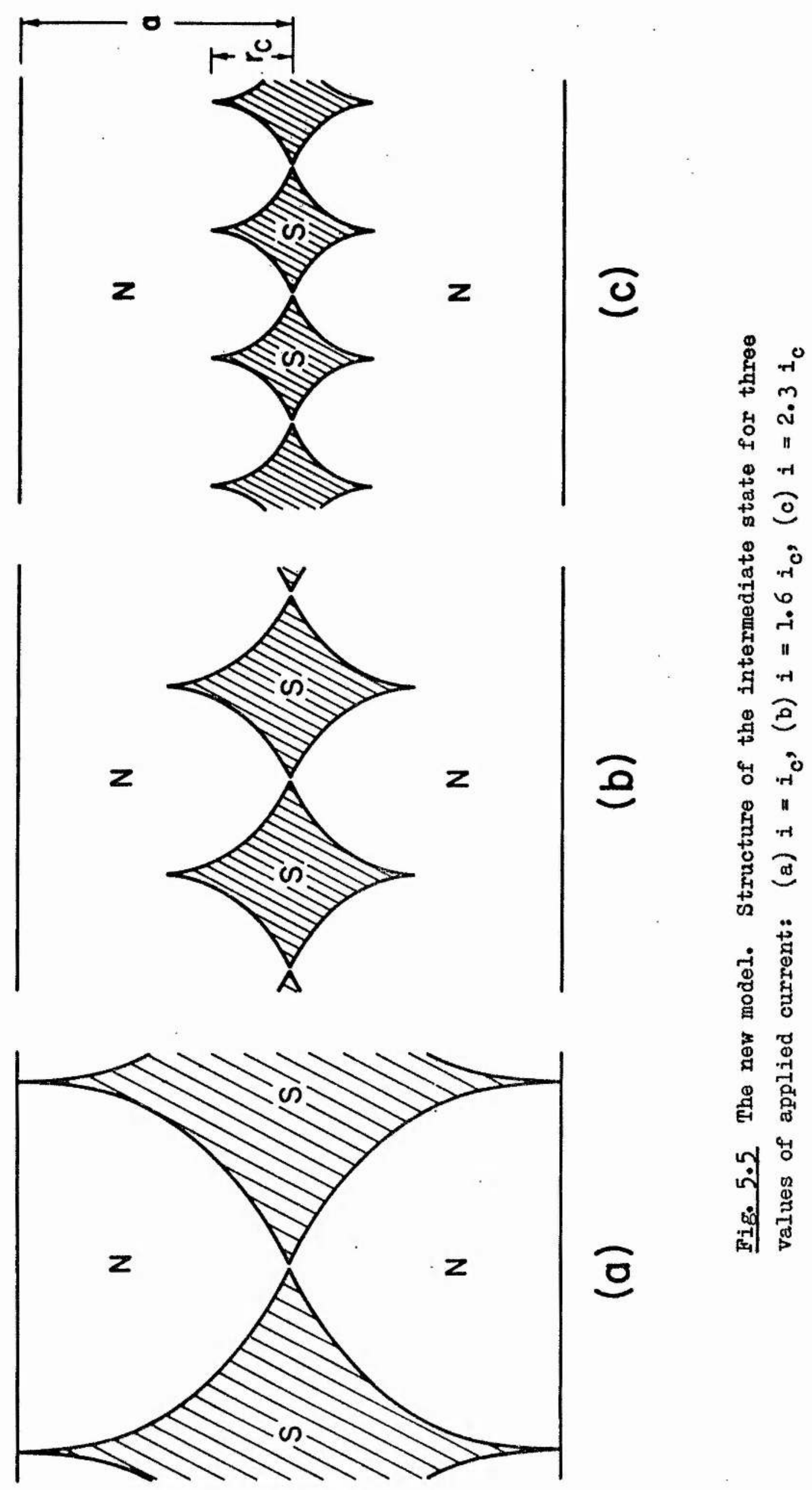




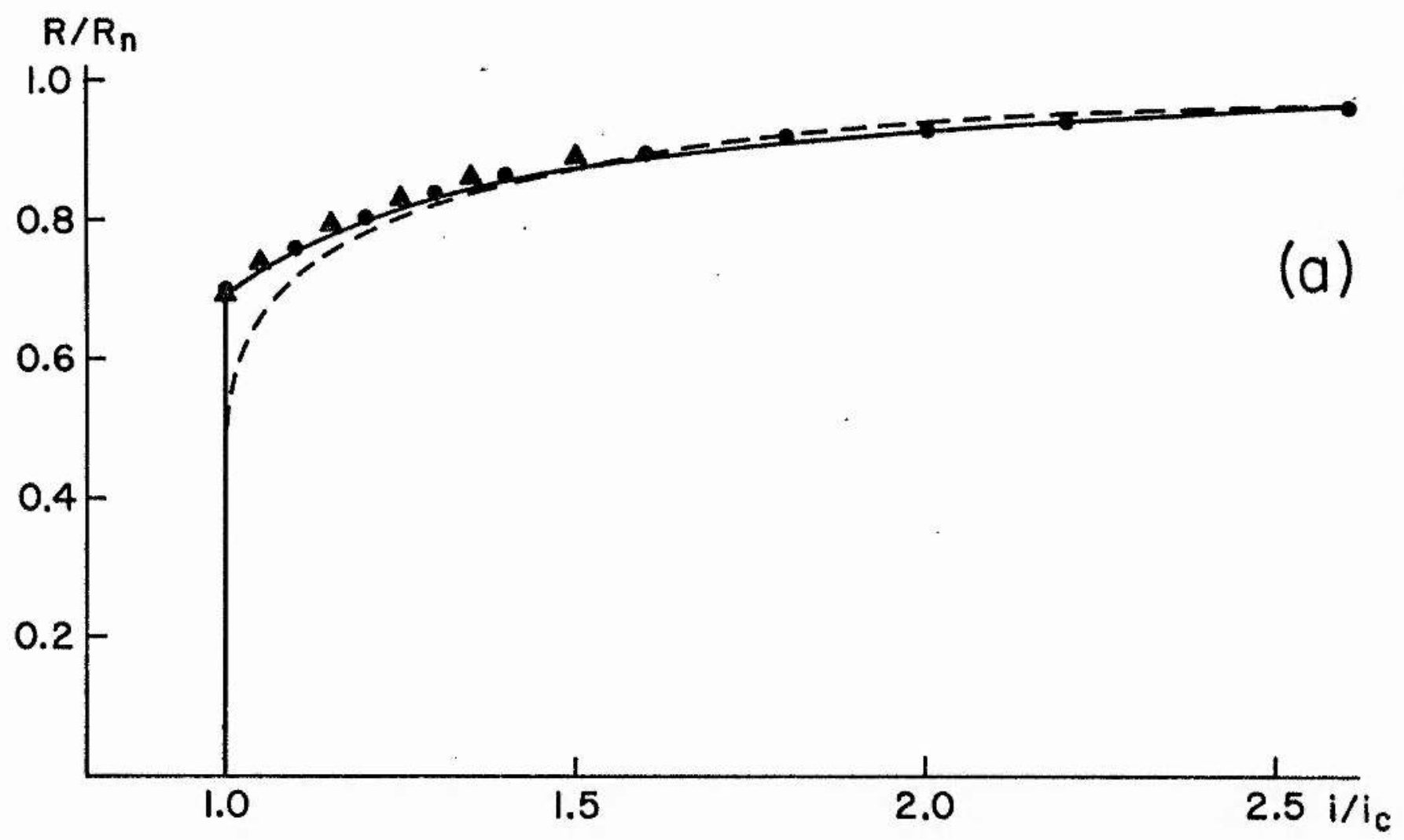

Fig. 5.6 Return of resistance curve ( $R$ is actual resistance, $R_{n}$ is fully normal resistance): solid line, this model; broken line, London's model; closed circles, our experimental values for $1.65 \mathrm{~mm}$. diametex Indium wire; closed triangles, experimental values for $3 \mathrm{~mm}$. diameter Indium wire (Freud et al, 1968)

secondary effects can occur). The table also shows the values given by London's model and Andreev's model (to be discussed later) and it can be seen that the predictions of our model are in reasonably good agreement with experimental results on Indium whereas the two other models do not give such good agreement. In comparing the values obtained by Freud et al for $3.0 \mathrm{~mm}$. diameter Indium wire with those obtained by us for $1.65 \mathrm{~mm}$ diameter Indium wire, it must be borne in mind that Freud ot al worked with very high currents. (ic $\sim 12$ amps. $)$

Table 5.3 also gives our experimental values for Thallium and, noting that these values are only accurate to about $3 \%$ (see page 3.22), it can be 


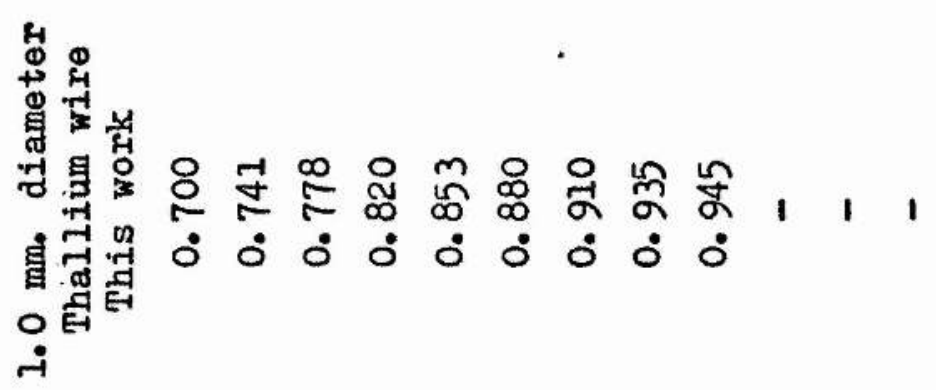

$$
\begin{aligned}
& \text { ఫ零 }
\end{aligned}
$$

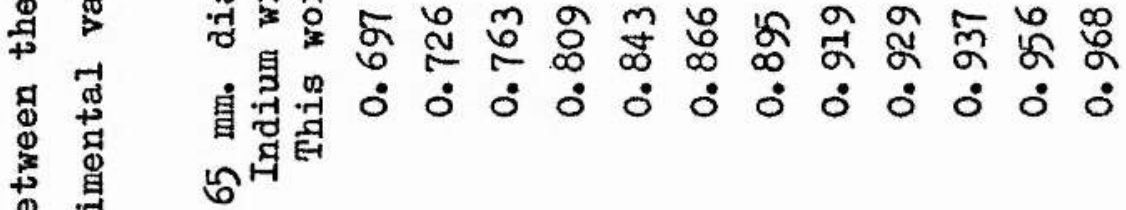

$$
\begin{aligned}
& \text { - } \\
& \text { : } \stackrel{0}{0}_{0}
\end{aligned}
$$

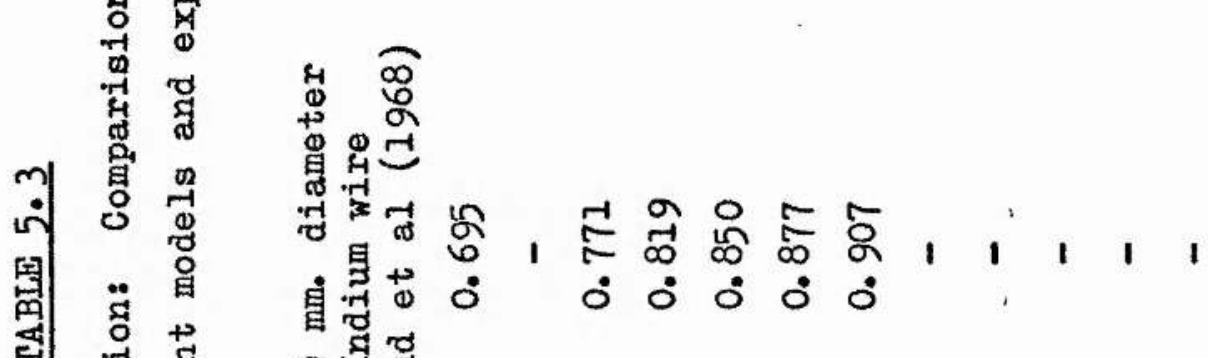

$$
\begin{aligned}
& \text { 官范 }
\end{aligned}
$$

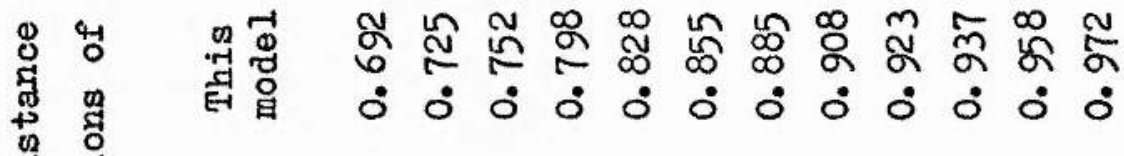

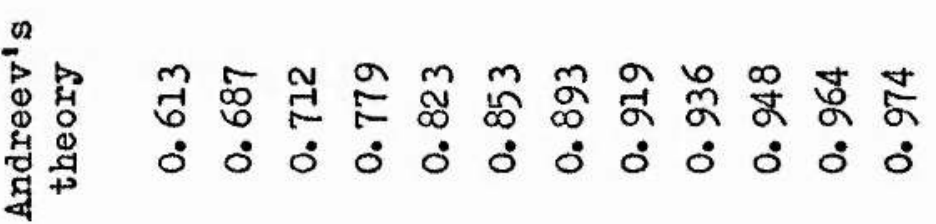

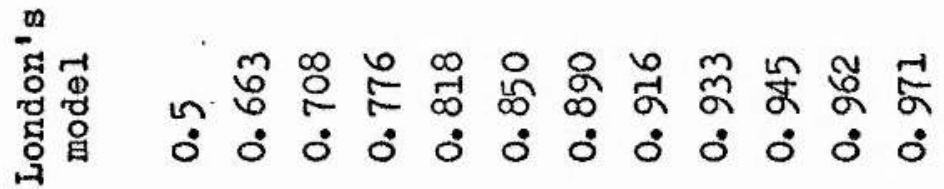

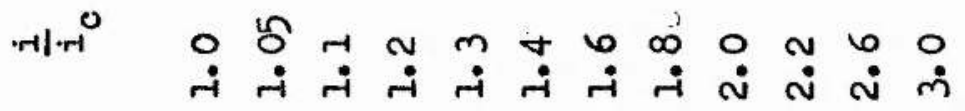


seen that they agree reasonably well with our model.

Experiments on tin have been carried out by a number of workers, for example Rinderer (1956), Meissner (1958), and Freud et al (1968). Of these both Rinderer and Freud et al worked with polycrystalline specimens and Meissner used both single crystal and polycrystalline specimens. Their results do not agree very well with each other and it is difficult to establish general qualitative trends which could perhaps be caused by secondary effects. For his purest specimen $\left(R_{3.8} / R_{300}=2.0 \times 10^{-4}\right.$, diameter $\left.=0.75 \mathrm{~mm}.\right)$ Rinderer does not get a sharp transition below about 7 amps. when he gets a value of about 0.6 for $\rho$. Freud et al used very pure $\left(R_{4.2} / R_{300}=6.4 \times 10^{-5}\right)$ but thick wire (diameter $=3.0 \mathrm{~mm}$ ) ) but even for currents upto $10 \mathrm{Amps}$. their results do not shcw a sharp transitiond Meissner worked with a large number of specimens of varying purity and diameters but his results vary to an extent that makes positive conclusions rather difficult. On the value of $\rho$ for tin Meissner's own conclusion is 'on the whole, the result of these measurements is that for ideally pure tin, $\rho$ depends only slightly on the diameter between $\alpha=0.1 \mathrm{~mm}$. and $d=3 \mathrm{~mm}$. and has values $0.57<\rho<0.73 .{ }^{\prime}$

Fink (1959) studied resistance transitions in Tantalum wires and found that they were in qualitative and quantitative disagreement with London's model. It is now known that his results are typical of Type-II behaviour and that Tantalum of the same purity as used by Fink is indeed a Type-II superconductor (see Bots et a.l, 1965).

5.5 COMPARISION WITH FXPERIMENT. RADIUS OF THE INTERMEDIATE STATH CORE:

An independent check on the validity of any model of the current-induced intermediate state is provided by Rinderer's (1956) measurements of the radius of the intermediate state core as a function of applied current. Rinderer considered the effect of a steadily increasing transverse field $\mathrm{H}_{t}$ on the intermediate state in a wire. Fig. 5.7(a) represents a diametral cross- 


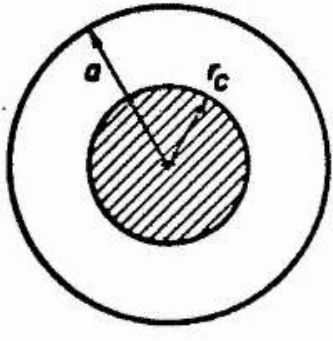

(a)

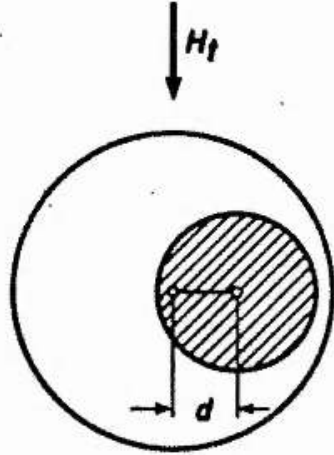

(b)

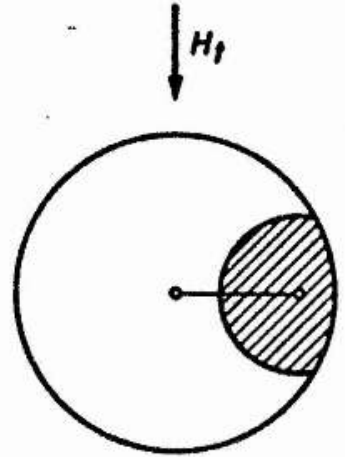

(c)

Fig. 5.7 The effect of a transverse magnetic field on the current-induced intermediate state in a wire specimen. The intermediate state core is shaded. (Based on Rinderer, 1956)

section of a wire which has a current greater than critical flowing through it; the shaded region is the intermediate state core of radius $r_{c}$ outside of which is the fully normal sheath. The only magnetic field present is the circumferential field $H_{i}$ produced by the current. If now a transverse field $\mathrm{H}_{t}$ is applied to the wire in the direction shown in Fig.5.7(b), the effect will be to increase the absolute value of the magnetic field in the left half of the cross-section and to decrease it on the right half, and hence the core moves to the right. Rinderer found that as $\mathrm{H}_{t}$ is increased the resistance of the wire does not change appreciably until a specific value $\mathrm{H}_{t_{0}}$ is reached at which point the resistance begins to increase presumably because the core begins to disappear as shown in Fig.5.7(c). From values of $\mathrm{H}_{\mathrm{t}_{\mathrm{c}}}$ for different values of $i / i_{c}$ Rinderer could calculate the corresponding values of $x_{c} / a$. Fig. 5.8 shows that the values obtained by Rinderer are in good agreement with the present model whereas they do not agree well with the values predicted by London.

5.6 SECONDARY EFFECTS. I. JOULE HEATING:

In the previous paragraphs of this chapter we have introduced a new model of the intermediate state and we have shown that the basic resistance transition 


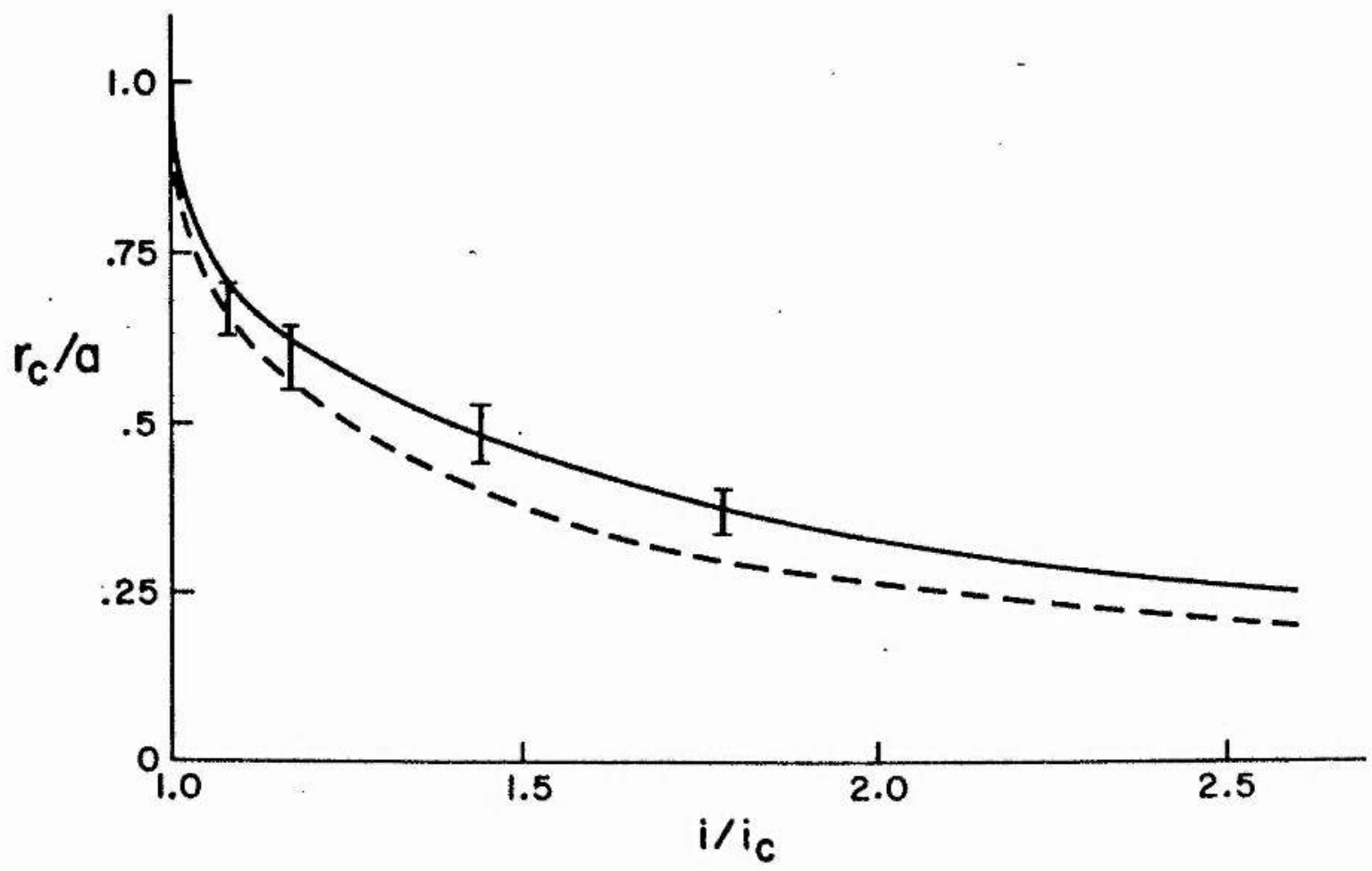

Fig. 5.8 Variation of the radius $r_{c}$ of the intermediate state core as a fraction of wire radius $a$, with applied current: solid line, this model; dashed line, London's model; experimental points are those obtained by Rinderer (1956)

predicted by the model is in reasonably good agreement with experimental results for thick wires. However in \$3.6 we have seen that the value of the rsistance jump $p$ depends on such factors as diameter of the wire and the actual value of the critical current. We shall now consider how these effects may arise.

Following London, we showed in \$2.2 that, ideally, the current distribution within any normal region of the intermediate state should satisfy the condition

$$
J(x) \propto 1 / x
$$

which implies a very high current density near the axis of the wire. Although the current density distribution in the present model does not quite satisfy this ideal condition, it is similar, as shown in Fig. 5.9 where the current density $J$ has been plotted as a function of the distance $r$ from the axis along a line in the centre of the normal region (e.g. PR in Fig.4.2 page 4.4) It follows that the major contribution to the joule heating occurs near the 


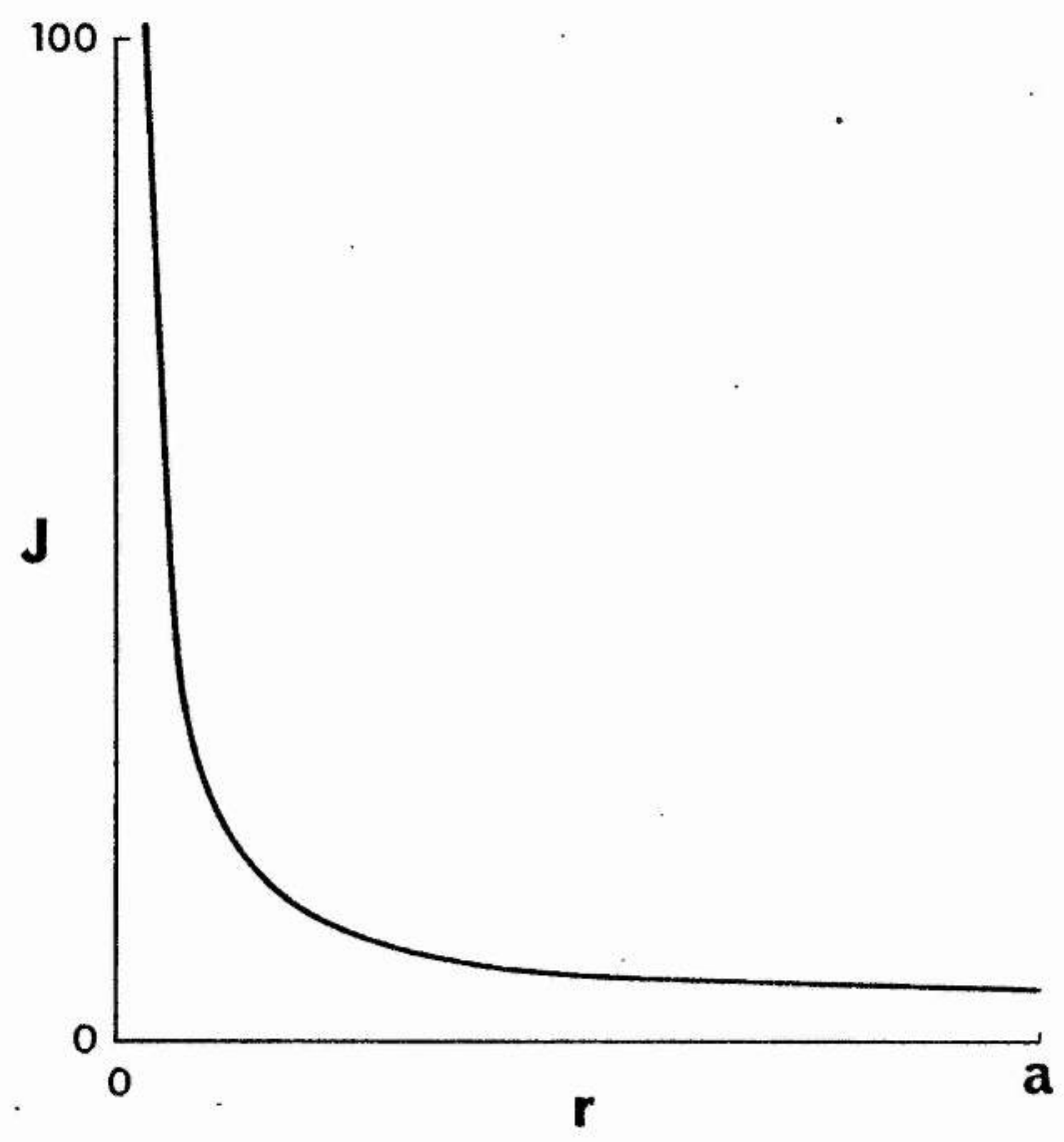

Fig. 5.2 The new model. Variation of current density with radius along a radius at the centre of the normal region.

axis of the wire. However in any actual experiment the specimen is cooled by immersion in a Helium bath so that the cooling takes place from the surface of the wire inwards. Thus, even at equilibrium, it is reasonable to expect a thermal gradient in the wire with the axis being at a higher temperature than the surface. A calculation of the temperature gradient involves a large number of factors:

(a) The Kapitza thermal boundary resistance at the surface of the specimen. Experimental evidence indicates that the Kapitza resistance is strongly dependant on the state of the surface (see e.g. Pollack, 1969).

(b) The thermal conductivity of the metal in the normal state.

(c) The thermal conductivity of the metal in the superconducting state.

(d) The thermal resistivity across an interphase boundary. 
(e) The shape of the interphase boundaries in the intermediate state:

(f) The current density distribution.

Neither the shape of the boundary nor the current density distribution are known in analytic form so that numerical methods would have to be used. Thus a calculation of the temperature distribution across the wire is a major computational undertaking and outside the scope of this work. We shall : therefore restrict ourselves to a qualitative discussion of the effects of joule heating.

Since the critical field $\mathrm{H}_{c}$ is a function of temperature, a thermal gradient in the wire with its axis being at a higher temperature than its surface implies a variation in the value of $H_{c}$ with distance $r$ from the axis, $\mathrm{H}_{c}$ being smaller near the axis than at the surface. In formulating our model we have used the criterion that $H_{c}$ be constant along the interphase boundary. In the course of the computational work involved in obtaining the best boundary, we have found that if the criterion is relaxed so as to allow the value of magnetic field on the interphase boundary to decrease near the axis, then the boundary shape is modified so as to increase the axial width w of the normal regions and the resistance of the resulting structure is higher than that given by our model. Thus the effect of joule heating is to increase the resistance of the intermediate state and, in particular, the value of the resistance jump $p$ at $i_{c^{*}}$ As a specific example we have found that if $H_{c}$ at the axis is about $25 \%$ less than its value on the surface of the wire, this results in an increase of about $8 \%$ in the value of $p$.

Joule heating is proportional to the resistance of the specimen and to the square of the current flowing through it. Hence for very low current transitions only a negligible amount of heat will be produced with little effect on the resistance transition. For transitions at higher values of critical current joule heating would become significant and we would expect that the value of $p$ will increase as $i_{c}$ is increased. Since the resistance of a wire 
specimen is inversely proportional to its crossection, we would expect the effects of joule heating to show at lower currents for wires of smaller diameter. Further since the presence of impurities increases the resistivity of specimens, we would expect impure specimens to show the effect of joule heating to a greater degree than would be shown by pure specimens of the same diameter.

In qualitative terms the above discussion agrees very well with the conclusions drawn in \$3.6 (page 3.18) on the basis of our experimental results.

\subsection{SECONDARY EFFECTS. II. THE SIZE EFFECT:}

In our method of calculating the resistance of any intermediate state structure (described in \$4.6) we have assumed that Ohm's Law holds good in the normal regions but we have neglected the possibility of electrons being scattered at successive interphase boundaries. Such scattering was first suggested by Kuper (1952) and would become important when the separation between successive interfaces is comparable to or smaller than the mean free path of electrons in the normal phase. Since in the present model the periodicity of the structure is directly proportional to the radius of the wire, it follows that scattering of electrons at interphase boundaries would only become important for relatively thin wires, and, following Kuper, we shall call this the'size effect'. The effect of the additional scattering would be to enhance the resistance of the wire in the intermediate state over the value calculated purely on the basis of Ohm's Law scattering. Thus we would only expect thick wires to have resistance transitions in agreement with the predictions of the present model. For 'thin' wires the resistance values should be higher and this expectation is confirmed, qualitatively, by our experimental results as shown in Fig. 3.17 and discussed in $\$ 3.6$ (page 3.17) and by those of other workers in the field.

Because of the geometry of the intermediate state structure in the present model, an exact calculation of the additional resistance due to interphase 
boundary scattering is impracticable. Even for the more simple London model Kuper had to use a 'crude approximation' and the following calculation is adapted from his treatment.

We assume the effective conductivity $\sigma$ to be a point function and we write

$$
\sigma=\sigma_{0} \frac{\mathrm{L}}{I}
$$

where $\sigma_{0}$ and 1 are the conductivity and electron mean free path respectively in bulk normal metal and $I$ is a quantity which we may call the 'effective mean free path' in a normal region of the intermediate state structure. For any given point $P$ in such a region, I may be eveluated as follows:

We construct a sphere of radius 1 about the point $P$. Let $\lambda$ be the. distance from $P$ to the surface of the sphere or the interphase boundary whichever is the smaller. Then

$$
I=\frac{1}{4 \pi} \int \lambda \cdot d \omega
$$$$
\text { -. } \quad \text {. }
$$

where $d \omega$ is the element of solid angle with $P$ as vertex. Since our interphase boundaries are not analytically defined, it is not possible to evaluate the above integral. Instead we may, once again, use a numerical approach and write

$$
L=\frac{1}{i} \sum_{i} \lambda_{i}
$$

where the $\lambda_{i}$ can be numerically evaluated along $i$ different directions. In our calculations we have used 60 such directions for each point $P$.

Our method of calculating the resistance of the intermediate state has been described in $\mathbf{0} 4.5$ where we showed that the ratio of the intermediate state resistance to that of the normal state is given by

$$
\frac{R_{I}}{R_{N}}=\frac{I_{N}}{I_{I}}
$$

where $I_{I}$ and $I_{N}$ represent the values of total current passing across a diametrai cross-section of the wire in the intermediate and normal states respectively under the same applied voltage. In calculating the values of $I_{N}$ and $I_{I}$ we had 
assumed that the normal phase conductivity was the same in both cases. Due to the size effect this is not strictly correct and the calculations may be repeated with the difference that in evaluating $I_{I}$ the current flowing across each mesh element has to be multiplied by the factor $\frac{\sigma}{\sigma_{0}}$ appropriate to that element and calculated as described above. In this way a corrected value of the resistance jump $\rho$ can be evaluated for any given mean free path $l$ and wire radius a.

It should be emphasised that the above method of correcting $\rho$ to take account of the size effect is only a crude approximation. In particular we have restricted ourselves to calculating $I / 1$ only for mesh points along the cross-sectional plane RP (see Fig.4.2, page 4.4) and have used this value as the correcting factor. A truly accurate calculation would require the correction factor to be evaluated and applied along every point of each current line in the normal region.

However, our approximate calculations are useful in giving a rough picture of what is happening and in Fig.5.10 (next page) we show a curve of $\rho$ vs. wire diameter for very pure Indium assuming $l=0.1 \mathrm{~mm}$. which is derived from the $\sigma / 1$ value of $9.0 \times 10^{10} \Omega^{-1} \mathrm{~cm}^{-2}$ obtained by Lyall and Cochran for 69 grade Cominco Indium; experimental values of $\rho$ obtained by us and other workers are shown for comparision. 


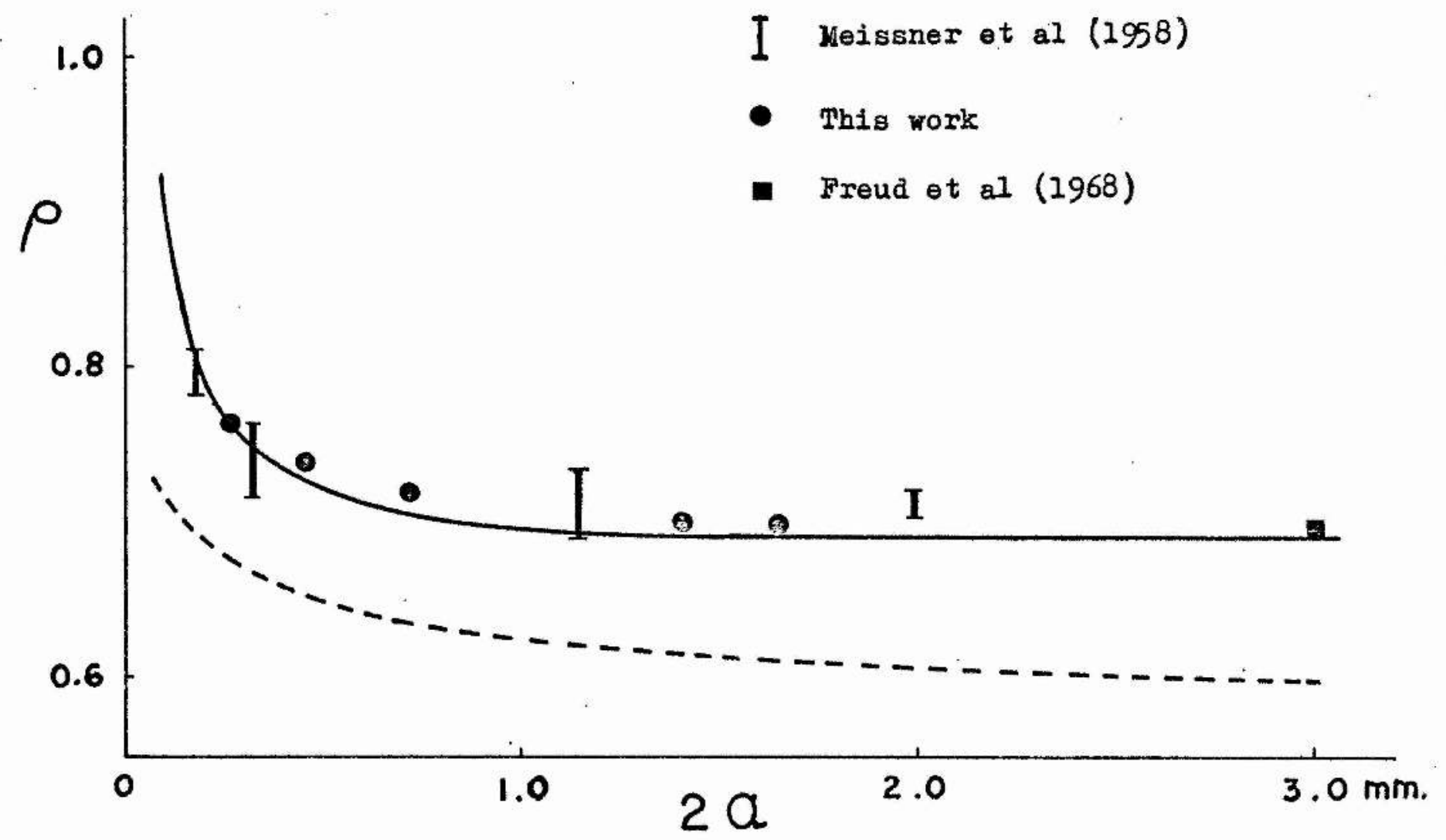

Fig. 5.10 Variation of the resistance jump $\circ$ as a function of the wire diameter 2a: solid line, this model; broken line, Andreev's theory; sources of experimental points are as indicated in the figure. 


\section{ANDREEV'S THEORY}

\section{1 ANDREEV'S MODEL:}

During the course of this work an alternative theory for the destruction of superconductivity by a current was put forward by Andreev (1968A). We find Andreev's paper rather hard to follow in places but we shall attempt to describe his approach and to compare his theoretical predictions with experimental values.

Andreev defines average values $\mathrm{H}$ and $\mathrm{E}$ of the magnetic and electric fields in the wire and writes the macroscopic electromagnetic equations which must be satisfied in the intermediate state in terms of these averaged quantities. The structure of the intermediate state will be given by the solution to these equations and Andreev obtains a family of solutions which are a function of an arbitrary constant $\alpha$. The structures thus obtained are not stationary but have a velocity which has components in both the axial and radial directions. For $\alpha=0$ the London structure is obtained and when $\alpha$ tends to infinity the Andreev theory leads to the Gorter model. The paraneter $\alpha$ corresponding to the equilibrium structure is determined by minimising the free energy and Andreev gets $\alpha=1.3$ in the limiting case of materials with a small Hall constant. Both the London and Gorter structures have higher free energies but Andreev points out that the potential of the London structure only exceeds that of his optimum structure by about $1 \%$, and that 'friction forces' due to inhomogeneities and crystal defects will reduce the optimum value of $\alpha$ and the structure will approach the London structure. For $\alpha=3.3$ Andreev finds the periodicity of his structure to be given by

$$
2 d \sim 2.9 \mathrm{a}^{\frac{2}{3}} \Delta^{\frac{1}{3}}
$$

and the velocity in the axial direction to be

$$
|u| \sim-0.1\left(c^{2} / \sigma a\right)
$$$$
\text { .. } \quad \text {.. }
$$

where $\Delta$ is the surface energy parameter and $\sigma$ is the normal state conductivity for the specimen. Using the above expressions, Andreev predicts a 
resistance transition of the form:

$$
\frac{R}{R_{n}}=\frac{1+\left(1-i^{1}-2\right)^{\frac{1}{2}}}{2}+\frac{1}{12 i} \cdot \frac{a}{\left(a^{2}-r_{c}{ }^{2}\right)} \cdot \frac{4 a^{2}}{r_{c}\left(1+\alpha^{2}\right)}
$$

where $i^{\prime}=i / i_{c}$ and $r_{c}=a\left\{i^{\prime}-\left(i^{\prime 2}-1\right)^{\frac{1}{2}}\right\}$

is the core radius as given by London.

Further, Andreev finds the resistance jump $\rho$ at $i=i_{c}$ to be related to the radius of the wire by the relation:

$$
\rho=\frac{1}{2}+0.64\left(\frac{\Delta}{a}\right)^{2 / 9}
$$

\subsection{COMPARISION WITH EXPERIMENT:}

Comparision of Andreev's theory with experiment is possible through the resistance transition and the variation of $p$ with wire diameter.

Andreev's prediction for the resistance as a function of current is given by equation (6.3) but it needs to be pointed out that this expression has an analytic discontinuity at $i=i_{c} ;$ for $i=i_{c}$ or $i^{\prime}=1, r_{c}=$ and the second term on the right hand side becomes infinite! Presumably we may use equation $(6.4)$ to get the value of resistance at $i=i_{c}$

We have calculated the resistance transition as pxedicted by Andreev's theory for Indium wire of $1.65 \mathrm{~mm}$. diameter using equation (6.3) for i'> 1 and equation $(6.4)$ for $i^{\prime}=1$. The values obtained are given in the third column of Table 5.3 (page 5.11) and these values may be compared with the predictions of our model and with our experimental values for $1.65 \mathrm{~mm}$ diameter Indium wire. It can be seen that Andreev's theory does not agree particularly well with experiment. Indeed comparisfon with the values given by London's model shows that for $i^{\prime}>1.1$ Andreev's values exceed London's predictions by only about $\frac{1}{2} \%$.

In so far as the radius of the intermediate state core is concerned, Andreev's theory does not differ from London's model and we have already shown 
(Fig5.8 page 5.14) that Rinderer's experimental values of the core radius agree with our model but not with London's.

The variation of the resistance jump $\rho$ with diameter of wire as given by Andreev has been evaluated for Indium and is compared with experimental results in Fig.5.10 (page 5.20). It would appearthat our model together with the correction applied to it due to the size effect agrees better with experimental values than does Andreev's theory.

Finally we note that for the case of Indium wire of $1.65 \mathrm{~mm}$. diameter, Andreev's theory gives a value of $0.178 \mathrm{~mm}$. for the periodicity of the intermediate state structure. The structure itself should be moving in the axial direction with a velocity of about $1.6 \mathrm{~cm} . / \mathrm{sec}$.

\subsection{THEORETICAL CONS IDERATIONS :}

In constructing his theory Andreev has formulated the equilibrium conditions in the intermediate state in terms of average values of the magnetic field. We doubt if this is a valid approach. Whether any point in the wire specinen should be in the normal or the superconducting state will surely be determined by the magnetic field at that point and not by an average of the field values along the length of the wire. The magnetic field at any point will be determined by the current distribution which is itself uniquely specified by the structure and the boundary conditions.

In his paper, Andreev (1968A) does not give any indication of what the equilibrium structure actually looks like. In presenting a paper at the XIth International Conference on Low Temperature Physics held at St. Andrews in 1968, Andreev (1968B) included a slide which showed the interphase boundaries to be fairly macroscopic paraboloids of revolution but this picture was not published in the proceedings. Since the interfaces shown were curved, it follows that the current lines in the normal regions would be curved and hence the field would not be constant throughout the normal region for the same 


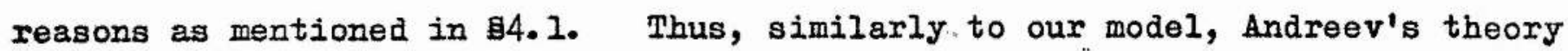
too calls for a certain amount of supercooling but this problem has not been discussed in his paper. 


\section{SUWMARY AND CONCLUSIONS.}

This work was started with the object of obtaining a better understanding of the resistance transition which occurs when superconductivity is destroyed in Type-I wires by pașage of a large enough current.

The two theories put forward by London (1937) and Gorter (1957) did not Five quantitative agreement with experimental measurements of the resistance transition. Besides, Shalnikov's experiments (1957) showed that the mechanism suggested by Gorter did not actually occur. Our own careful measurements of the resistance transition for a series of Indium wires and a Thallium specimen showed that London's model was indeed quantitatively inadequate.

We then investigated Iondon's theory more closely from a theoretical point of wiew and found that the intermediate state structure which he had suggested did not satisfy the conditions necessary for the coexistence of normal and superconducting regions in thermodynamic equilibrium; we found that no finite structure could quite satisfy the conditions. This led us to formulate a slightly less 'ideal' criterion for the coexistence of the two phases in the intermediate state. Our now criterion required the magnetic field to have the critical value along the interphase boundaries but allowed for a certain amount of supercooling within the normal regions providing this was within the Iimits acceptable on the basis of theory and independent experimental observations.

Jsing numerical methods we found a series of intermediate state structures which all satisfied our criterion and by requiring the supercooling to be a

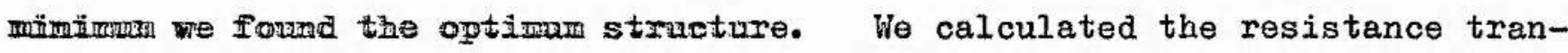
Sultion on the basis of our new model and found it to be in reasonable agreement wïtit experimental values obtained by us and other workers for 'thick' Indium and Thallium wires. 
An independent check on the validity of our model was provided when we found that the radius of the intermediate state core as given by our model agreed with the values obtained experimentally by Rinderer.

Bxperimental investigations had revealed that the resistance transition was a function of such factors as wire diameter and purity. We have shown that these variations could be understood, at least qualitatively and to some extent quantitatively, by considering the effects on our model of joule heating and of electron scattering at the interphase boundaries.

An alternative model put forward by Andreev (1968A) almost simultaneously with ours does not give good agreement with experimental results of the resistance transition and of the radius of the intermediate state core. As mentioned in the previous chapter, we are also not convinced that Andreev's approach is theoretically justified.

Thus the model presented in this work gives better agreement with experimental observations than any of the other models proposed and we may conclude that our work has improved our understanding of the destruction of superconductivity by a current. 


\section{REFERENCES}

Abrikosov, A. A., 1957. Sov. Phys. J.E. T.P. 2, 1174.

Andreev, A. F., 1968A. Sov. Phys. J.E. T.P. 27, 809.

Andreev, A. F., 1968B Proc. of the XIth Int. Conf. on Low Temperature Physics, University of St. Andrews, $2,831$.

Aziz, R. A. and Baird, D. C., 1959. Can. J. Phys. 37, 937.

Bardeen, J., Cooper, L. N. and Schrieffer, J. R. 1957. Phys. Rev. 106, 162.

Phys. Rev. 108, 1175.

Berkovich, S. Ya, and Lapir, G. M. , 1963. Sov. Phys. J.E. T. P. 17, 896.

Bogoliubov, N. N. , Tolmachev, V. V. and Shirkov, D. V. 1958. A new method in the theory of Superconductivity. Academy of Science, Moscow.

Bots, G. J. C. , Pols, J. A. , Blaisse, B. S. , De Jong, L. N. J. and Van Engelen, P.P. J. 1965. Physica 31, 1113.

Cooper, L. No, 1956. Phys. Rev. 104, 1189.

Daunt, J. G., Miller, A. R., Pippard, A. B. and Shoenberg, D., 1948 Phys. Rev. 74, 842. De Gennes,P.G., 1966. 'Superconductivity of Metals and Alloys', W. A. Benjamin Inc., New York.

De Haas, W.J. and Voogd, J., 1931. Leiden Communication 214c

Deltour, R., Delang, H. N. and Wyder, P., 1970. Phys. Letts. 31A, 515.

Eilenberger,G., 1966. Z. Phys. 190, 142.

Faber, T.E., 1957. Proc. Roy. Soc. A241, 531.

Fink, H. J., 1959. Can. J. Phys. 37, 485.

Freud,R., Sulkowski, Cz. and Makiej, B., 1968. Phys. Letts. 27A, 187.

Freud,R., Makiej,B. and Sikora,A. 1968A. Phys. Stat. Sol. 25, K127.

Frohlich,H., 1950. Phys. Rev. 79, 845. .

Ginzburg, V. I., 1956. Sov. Phys. J.E.T.P. 3, 621 and 4, 594.

Ginzburg, V. L. and Landau, L. D., 1950. J.Exp. Theor. Phys. (USSR) 20, 1064.

Gorkov, I. P., 1959. Sov. Phys. J. E. T.P. 2, 1364.

Gorter, C. J., 1957. Physica 23, 45.

Gorter, C. J. and Casimir, H. B. G., 1934. Phys. Z. 32, 963.

Gorter, C.J. and Potters, M. L., 1958. Physica 24, 169. 
Kuper, C. G., 1952. Phil. Mag. 43, 1264.

Kuper,C.G. and Tait,W., 1967. private communication.

London, F., 1935. 'Une conception nouvelle de la supraconducticilite', Hermann et Cie., Paris.

London, F. and London,H., 1935. Proc. Roy. Soc. Al55, 71.

Lucas, G. and Stephen, M. J., 1967. Phys. Rev. 154, 39.

Iynton,E. A., 1969. 'Superconductivity', Methuen and Co. Ltd., London.

Hatthias, B. T., 1969 in 'The NicGill University Advanced Study Institute on Superconductivity: (Gordon and Breach, New York).

Maxwell,E., 1950 Phys. Rev. 18, 477.

hoGill, N. C., 1968. private communication.

Meissner, H. 1958. Phys. Rev. 109, 668.

Meissner, H. and Zdanis, R., 1958. Phys. Rev. 109, 681.

Heissner, H. and Ochsenfeld,R. 1933. Naturviss. 21, 787.

Neighbor, J.E. , Cochran, J.F. and Shiffman, C. A., 1965. in 'Low Temperature Physics, IT9', (Plenum Press, New York) page 479.

Onnes,K., 1911. Leiden Communications 1206, and 1226

Pippard,A. B., 1953. Proc. Roy. Soc. A216, 547.

Pollack, G. I., 1969. Rev. Mod. Phys. 41, 48.

Quinn, D. J. and Ittner, W. B., 1962. J. Appl. Phys. 33, 748.

Reynolds, C. A., Serin, B., Wright, W. H. and Nesbitt, L. B., 1950. Phys. Rev.78, 487. Rinderer, I., 1956. Helv. Phys. Acta. 22, 339.

Rose-Innes, A. C., 1959. Brit. J. App. Phys. 10, 452.

Schawlow, A. L., 1958. Phys. Rev. 109, 1856.

Scott,R. B., 1948. Bur. Stand. J. Res. 41, 581.

Silsbee, F. B., 1916. J. Wash. Acad. Sci. 6, 597.

Shalnikov, A. I., 1957. J. Exptl. Theor. Phys. (USSR) 33, 1071.

Shoenberg, D., 1952. 'Superconductivity'. (Cambridge U.P., London)

Troinar,E., 1960. Sov. Phys. J.E.T.P. 11, 470.

Van Mal,H.H. and Akerboom,F., 1968. J. Sci. Instr. (Ser.ii) 1, 689。

Lyall, K. R. and Cochran, J. F., 1967. Phys. Rev. 159, 517. 


\section{ACKNOWLEDGEMENTS}

I would like to express my grateful thanks to the following people for their help during this work.

Professor J.F. Allen, F.R.S., for suggesting that I work in the field of superconductivity, for advice and encouragement while the work was being carried out and for having created a department with a friendly atmosphere in which it was such a pleasure to work.

Dr. D. C. Baird, for suggesting the project, for his advice and assistance all through this work and for his infectious enthusiasm.

Professor A.J. Cole and his staff at the St. Andrews University Computing Laboratory for their assistance with the numerical calculations.

Mr. R. H. Mitchell, for his invaluable advice and assistance on all cryogenic matters.

Mr. Howard Cairns for always lending apparatus in good cheer.

Mr. J. McNab and Mr. I. Robertson and his staff at St. Andrews for teaching me what little I know of workshop practice and for making the apparatus.

Mr. E. Redman, for always providing for my 'urgent' requirements with a smile on his face.

Last, but not least, my wife Ruma, for her encouragement and understanding while this work was being completed. 


\section{A_TYPICAL_COMPUTERE_PROGRAMME}

We give below an example of the computer programmes used to find the most satisfactory interphase boundaries for the current-induced intermediate state. The various steps involved have been described in Chapter 4 . The programme is written in FORTRAN language and headings in the body of the programme describe the various phases of the calculation.

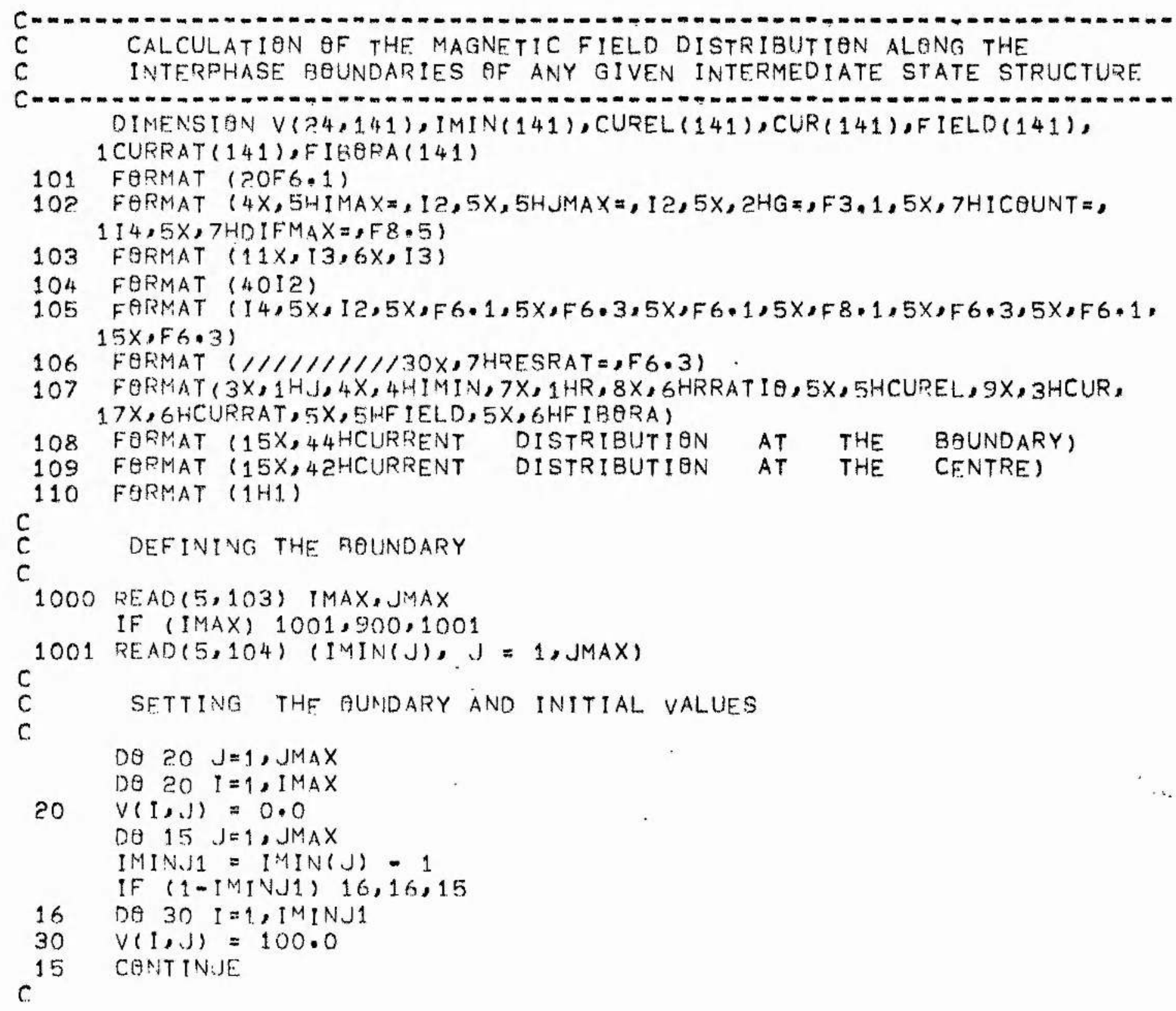


ICAUNT $=0$

$G=0.8$

IMAXI $=I M A X-1$

JMAX $1=J M A X-1$

100 DIFMAX $=0.0$

IMINI $=\operatorname{IMIN}(1)$

IF (IMAX-IMINI) 35,35,25

25 DO $160 \quad I=I M I N 1, I M A \times 1$

$V A L=(V(I+1,1)+V(I-1,1)+4 \cdot 0 * V(I, 2)) / 6.0$

DIFF $=V A L-V(1,1)$

IF (DIFF-DIFMAX) $160,190,190$

190 DIFMAX $=$ DIFF

$160 V(I, 1)=V A L+G * D I F F$

35 DQ $45 J=2, J M A X 1$

$R=J-1$

$B=1.0+0.5 / R$

$C=1.0-0.5 / R$

$\left.I M I N_{1}\right\rfloor=\mid M I N(J)$

IF (IMINJ-IMAXI) $36,36,45$

36 DE $40 \quad I=I M I N J, I M A X 1$

$V A L=0,25 *(V(I+1, J)+V(I-1, J)+B * V(I, J+1)+C * V(I, J-1))$

$D I F F=V A L-V(1, J)$

IF $(D I F F-D I F M A X) \quad 40,60,60$

60 DIFMAX = DIFF

$40 \quad V(I, J)=V A L+G * D I F F$

45 CENTINUE

DO $70 \quad I=1$, IMAX

$70 V(I, J M A X)=V(I, J M A X 1)$

ICOUNT $=$ ICOUNT +1

IF (DIFMAX -0.001$) 90,100,100$

$\mathrm{C}$
$\mathrm{C}$
$\mathrm{C}$

PRINT BUT OF THF ELECTRIC PATFNTIAL DISTRIBUTION

90 WRITE $(6,110)$

WRITE(6,102) IMAX, JMAX, G, ICOUNT, DIFMAX

IF $(I M A X-? 0) 1 ? 1,121,122$

12.1 DO $12.3 \mathrm{~K}=1$, JHAX $J=J M A X-K+1$

$123 \operatorname{WRITE}(6,101)(V(1,1), I=1, \operatorname{IMAX})$

GQ TO 150

122 DO $1 ? 4 \quad K=1, J M A X$ $J=J M A X-K+1$

124 WRIYE $(6,101)(V(1, J), I=1,20)$

WR ITE $(6,110)$

If $(M \wedge X-40) \quad 125,125,126$

125 DO $127 \mathrm{~K}=1$, JMAX

$J=J M A X-K+1$

127 WRITE $(5,101)(V(I, J), I=21, \operatorname{IMAX})$

GQ TO 150

126 DQ $128 \quad K=1$, JMAX $J=J M A X-K+1$

128 WRITF $(6,101) \quad(V(I, J), I=21,40)$

WR ITF $(6,110)$

129 DO $131 \mathrm{~K}=1$, JMAX $J=J M A X-K+1$

131 WRITE $(6,101) \quad(V(I, J), I=41, I M A X)$ 
c

CALCULATIAN EF CURRENT AND MAGNETIC FIELD DISTRIBUTION ALONG

C INAERDHASE RQUNDARY

138 CUREL(1) $=(100.0-$ VIMIN1.1))*0.25

DQ $400 \quad J=2, J M A X$

$R J=\rfloor$

$R=R J-0.5$

$I M I N J=I M I N(J)$

CUREL $(J)=(100 \cdot 0-V(I M I N J \cdot J)) *(2,0 * R-1 \cdot 0)$

IF (IMIN(J) - IMIN(J-1)) 410,420,420

410 IMINJ $=I M I N(J)$

IMINJM = IMIN $(J-1)-1$

De $430 \quad I=I M I N J$ S TMINJM

430 CUFEL $(J)=\operatorname{CUREL}(J)+(100.0-V(I, J)) * 2.0 *(R-1.0)$

420 CONTINUE

CUR (1) = CUREL (1)

$400 \operatorname{CUR}(J)=\operatorname{CUR}(J-1)+\operatorname{CUREL}(J)$

DA $450 \quad J=1$, JMAX

$R J=J$

$R=R J-0.5$

$F I E \perp D(J)=C U R(J) / R$

450 CURRAT (J) $=$ CUR $(N) /$ CUR (JMAX)

DA $445 \mathrm{~J}=1$, JMAX

445 FISGRA(J) = FIELD(J)/FIELD(JMAX)

WRITF $(6,110)$

WRITF $(6,108)$

WRITE $(5,107)$

DQ $440 \quad K=1$. JMAX

$J=J M A X-K+1$

$R J=J$.

$R=R J=0.5$

RJMAX = JMAX

RMAX = RJMAX -0.5

RRATIE = R/RMAX

440 WRTTE( 6.105) Je IMIN(J), R, RRATIE, CUREL(J), CUR(J), CURRAT(J) 1, FIELD(J), FIBSRA(J)

C

C.

C

$\operatorname{CUR}(J)=\operatorname{CUR}(J-1)+\operatorname{CUREL}(J)$

DQ $510 \mathrm{~J}=1$, JMAX

RJ $=J$

$R=R J-0.5$

FIELD $(J)=$ CUR $(J) / R$

510 CURRAT (J) $=\operatorname{CUR}(J) / C$ UIR $(J M A X)$

DE $515 \mathrm{~J}=1, J M A X$

515 FIPQRA(J) = FIELDI JIFIELD(JMAX)

WR ITF. $(k, 110)$

WRITF $(5,109)$

WRITF $(6,107)$

DQ $170 K=1$, JMAX 
$J=J M A X-K+1$

RJ $=J$

$R=R J-0.5$

RJMAX = JMAX

RMAX $=$ RJMAX -0.5

RRATI $\theta=$ R/RMAX

170 WRITE( 6,105j) J,IMIN(J),R,RRATIA,CUREL(J),CUR(J),CURRAT (J),FIELD( 1J), FIBARA (J)

c

CALCULATION AF RESISTANCE RATIO

$D=J M A X-1$

$E=I M A X=1$

$F=\operatorname{JMAX}$

RESRAT $=100,0 * F * D /(E * \operatorname{CUR}(J M A X))$

WRITE (6,106) RESRAT

GQ TO 1000

900 CALL EXIT

END 


\section{APPENDIX II}

\section{PUBLICATIONS BASED_ON THIS WORK}

In the following pages we present publications based wholly or partly on this work. In chronological order these are:

1. D.C. Baird and B.K. Mukherjee, (1967), Phys. Letts. 25A, 137.

2. B.K. Mukherjee, J.F. Allen and D.C. Baird, (1968) in 'Low Temperature Physics LTII' (University of St. Andrews, Scotland) 2, 827

3. D.C. Baird and B.K. Mukherjee, (1968), Phys. Rev. Letts. 21, 996.

4. B. K. Mukherjee, H. D. Wiederick and D.C. Baird, (1970) To be published in the Proceedings of the XIIth International Conference on Low Temperature Physics held at Kyoto in 1970.

5. D.C. Baird and B.K. Mukherjee, (1971), Phys. Rev. B. 3, 1043. 


\title{
DESTRUCTION OF SUPERCONDUCTIVITY BY A CURRENT
}

\author{
D. C.BAIRD * and B. K. MUKHERJEE \\ Department of Physics. University of St. Andrew's. St. Andrew's. Scotland
}

Received 12 June 1967

\begin{abstract}
A revised form of the London theory of the destruction of superconductivity by a current is presented. Numerical methods are used to find the optimum phase boundary configuration. The result specifies the scale of the structure and the resistance of the intermediate state.
\end{abstract}

When the current through a superconducting wire is gradually increased, a critical value $i_{\mathrm{c}}$ is reached at which resistance is restored dis continuously to some fraction $\rho$ of the normal resistance. The observed value of the critical current is generally in agreement with Silsbee's rule [1]. The present theory of the destruction of superconductivity by a current, put forward by London [2], predicts a value of 0.5 for $\rho$. However, experimentally $\rho$ has been found to vary in the range 0.6 to 0.8 , depending on parameters such as temperature and purity of specimen [3-6]. Attempts have been made to resolve this discrepancy by considering secondary effects such as heating of the wire [7] and electron scattering at the normal-superconducting phase boundary [8], but no attempt seems to have been made to correct what appear to be basic defects in the London theory. We present here a revised form of the theory which predicts for $p$ a value in excess of 0.5 and which also indicates the scale of the intermediate state structure.

When the current in a superconducting wire reaches a value such that the magnetic field at the surface has the critical value $H_{\mathrm{c}}$, flux should start to penetrate the wire, thus creating a nor mal region. Throughout this region the field must obviously be $H_{\mathrm{c}}$, or as close to $H_{\mathrm{C}}$ as possible, and it follows that the variation of current density with radius $r$ must have the form:

$$
j(r)=i_{\mathrm{c}} / 2 \pi a \cdot r
$$

where $a$ is the radius of the wire. Or, in terms of the current $i(r)$ within raclius $r$, the requirement in the normal region is that

* On leave from the Royal Military College, Kingston, Ontario, Canada.

$$
i(r)=i_{\mathrm{c}} r / a
$$

In the original presentation by London and in the description given by Shoenberg [9], it is assumed that the reguired current distribution will be produced by the phase boundary configuration illustrated by them [2, fig. $40 ; 9$ fig. 56]. However this is true only if the angle at the apex of each normal region is vanishingly small. It would be more satisfactory if one could find a finite interphase configuration which gives the required current distribution. This would have the advantage of specifying the intermediate state configuration uniquely and the resistance associated with the state would automatically follow.

The current distribution can be calculated from the potential distribution in the normally conducting region. This potential distribution is simply a solution of Laplace's equation subject to the boundary conditions which are (i) that $V=$

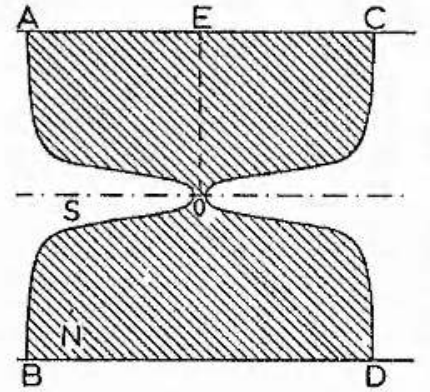

(a)

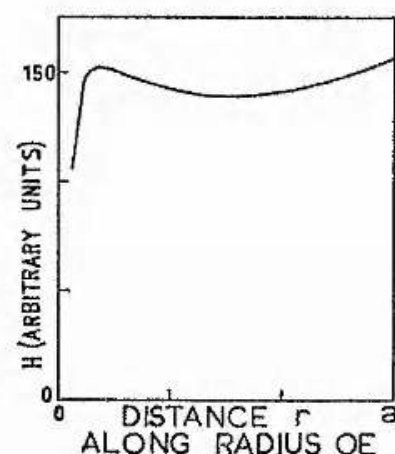

(b)
Fig. 1. (a) Axial section of the conductor showing the most favourable phase boundary. (b) The variation of field along radius of for the phase boundary shown in (a). 
constant on any $s-n$ boundary, and (ii) that $\mathrm{c} V / \partial r$ $=0$ at the surface of the wire. In a search for a phase boundary which will give the most nearly uniform distribution of field along the radius, we have used a relaxation method to obtain numerical solutions for a number of boundary configurations. The most satisfactory boundary shape which has emerged is shown in fig. 1(a), and the corresponding variation of magnetic field along the radius $O E$ is shown in fig. 1 (b). There is a major variation of field near the axis and a small departure from constancy away from the axis. The latter could almost certainly be removed by further slight adjustment of the boundary shape, which would be facilitated by the use of a finer computational mesh than the $30 \times 30$ mesh which we have used so far. The deviation near the axis is only to be expected since no finite computational mesh can reproduce the current density singularity at the centre implied by equation (1). Besides, this condition also entails a temperature singularity at the axis. Such singularities are physically unrealistic and it appears reasonable to expect an equilibrium situation with both the current density and temperature taking finite values at the axis.

It is important to note that fig. 1(a) effectively defines the scale of the intermediate state structure, the width $\mathrm{AC}$ being approximately equal to the diameter of the wire. The resistance associated with such a boundary configuration can be calculated in terms of the fully normal resistance. For the phase boundary of fig. 1(a), the ratio $\rho$ ' is 0.62 . It is found that the value of $\rho$ is very sensi- tive to variations of the critical field in the normal region and only a small percentage variation of field need be allowed for $\rho$ to give a value as high as 0.8 . Such small variations in the critical field could easily be caused by secondary effects such as Joule heating. In our treatment we have assumed that the mean free path of the electrons is small compared with the scale of the intermediate state structure. If this is not so, the effect will be to increase the resistance ratio $\rho$. We are currently investigating these effects and extending our treatment to cover the resistance curve for currents greater than $i_{\mathrm{c}}$.

, We wish to express our thanks to Professor J. F. Allen and to Dr. C. G. Kuper for many helpful discussions and suggestions, and to Dr.A.J. Cole and the staff of the St. Andrews University Computing Laboratory for their assistance with the numerical solutions.

$+$

References

1. F. B. Silsbee, Bull. Bur. Stand, 14 (1918-19) 301.

2 . ${ }^{\mathrm{T}} \mathrm{F}$.London, Superfluids Vol, 1 (John Wiley and Sons, Ino.,.,New York, 1950).

3. R. B. Scott. J. Res. Nat. Bur, Stand, 41 (1948) 581.

4. L. Rinderer, Helv. Phys. Acta 29 (1956) 339.

5. H. Meissner, Phys. Rev. 109 (1958) 668.

6. H. Meissner and R. Zdanis, Phys. Rev. 109 (1958) 681.

7. S. Y. Berkovich and G. M. Lapir, Soviet. Phys. JETP 17 (1963) 896 .

8. C. G. Kuper, Phil. Mag. 43 (1952) 1264.

9. D. Shoenberg, Superconductivity (Cambridge University Press, 1952). 
B4.4 DESTRUCTION OF SUPERCONDUCTIVITY BY A CURRENT.

B. K. Mukherjee and J. F. Allen, School of Physical Sciences, Jniversity of St. Andrews, St. Andrews, Scotland and D. C. Baird, Department of Physics, Royal Military College. Kingston, Ontario, Canada.

It is well known that, when the current through a superconducting wire is gradually increased, a critical value $i_{c}$ is reached at which resistance is restored discontinuously to some fraction $p$ of the normal resistance. As the current is increased further the resistance asymptotically approaches the normal value. The observed value of the critical current is generally in agreement with Silsbee's rule. In 1936 . F. London ${ }^{(2)}$ theoretically predicted a value for $p$ of 0.5 . However. observations $(3,4,5,6)$ generally give values for $p$ of between 0.7 and 0.9 . Proposals have been made to resolve this discrepancy by considering secondary effects such as heating of the wire ${ }^{(7)}$ and mean free path effects ${ }^{(8)}$. In pointing out ${ }^{(1)}$ that the model suggested by London was also not satisfactory theoretically, we presented a new treatment which accorded better with observation. We give here an extension of that treatment which covers not only the conditions at $i_{c}$ but also gives the resistance at higher currents.

When the current in a superconducting wire reaches a value such that the magnetic field at the surface has the critical value $\mathrm{H}_{\mathrm{c}}$, flux should start to penetrate the wire and create a series of normal regions along the wire. Throughout these regions the field must obviously be $\mathrm{H}_{\mathrm{c}}$ or as close to $\mathrm{H}_{\mathrm{c}}$ as possible. It follows that, in the normal regions, the variation of current density with radius $r$ should ideally have the form

$$
J(x)=\frac{1_{c}}{2 \pi a} \cdot \frac{1}{x}
$$

where $a$ is the radius of the wire. However, it is not possible to 
34.4

find a finite structure which gives $\mathrm{H}=\mathrm{H}_{c}$ throughout the whole of the romal volumes, and the most important condition is obviously that $\mathrm{H}=\mathrm{H}_{\mathrm{c}}$ at any normal-superconducting interface.

For a given boundary, the potential distribution in one of the normal regions can be obtained by a numerical solution of Laplace's equation subject to the boundary conditions

1) $\mathrm{V}=$ constant on any $s-n$ boundary, 2) $\frac{\partial V}{\partial r}=0$ at the surface of the wire. The current distribution and hence the field distribution can then be calculated from the potential distribution. Using trial and error methods a boundary satisfying the above condition can be found for any given ratio of radius to a structure perlodic length $d$ up to a limiting value $(a / d)_{\max }=1.4$. Some of these boundaries are show in Fig. 1. Beyond tris limiting value of $(a / d)_{\max }$ it has not been possible to find satisfactory boundaries. The criterion that throughout the normal region $\mathrm{H}$ be as ciose as possible to $\mathrm{H}_{\mathrm{c}}$ requires that

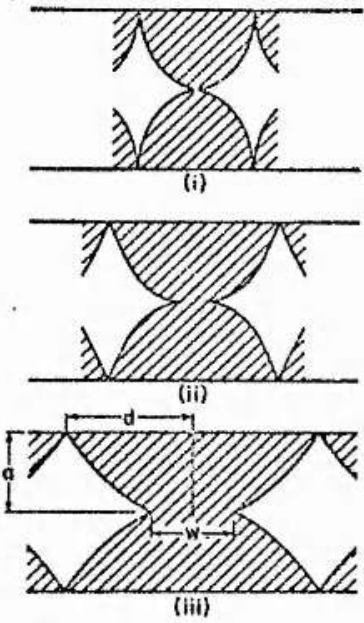

Fig. 1. Best boundaries for $a / d$ values (1) 1.4 (ii) 1.0 and (iii) 0.67

a/d has its maximum value, and we have chosen $a / d=1.4$ as the optimum proportion. Surface energy effects have been calculated and found to be negligible for values of $a / d$ of this order.

... ie resistance due to such a structure has been calculated ard we iind a value of $p=0.69$.

When the current rises above $1_{c}$, the superconducting cores must inrink to an equilibrium shape for each value of current so as to intrair. the condition $\mathrm{H}=\mathrm{H}_{\mathrm{c}}$ along the boundaries. Boundaries 
B4.4

829

to satisfy this criterion have been found for several values of $i>i_{c}$, and two of them are shown in Fig. 2. The resistances of these structures have been evaluated and the return of resistance curve as predicted by this model is shown in Fig. 3 .

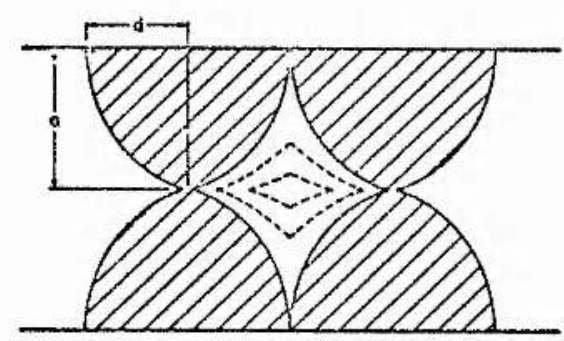

Fig. 2. Successive boundaries for $1 / i_{c}$ values of 1.0 , $1.31,2.2$.

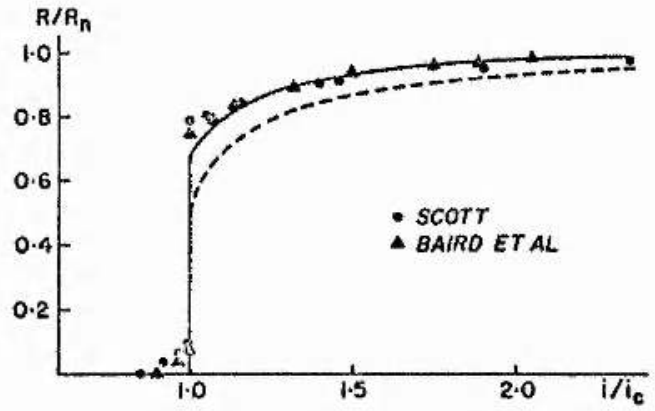

Fig. 3. Return of resistance curve: - - (present model). (Iondon), Scott $(0.286 \mathrm{~mm}$. diameter Incium wire), \& Baird et al $(0.5 \mathrm{~mm}$. diameter Indium wire).

Since the present model gives a definite size for the structure, it is amenable to the inclusion of secondary effects such as mean free path and joule heating.

In the above calculations we have assumed that the mean free path 1 in the normal material 1 s small compared with the dimensions of the normal regions. Kuper has suggested that when this is not the case, scattering of electrons at n-s interfaces will reduce the efiective mean free path, thereby increasing the value of $\rho$. He has proposed $^{(8)}$ that for the London model the average effective conductivity is

$$
\sigma(r)=\sigma_{0}[1-(1-s) \exp (-r s / e)]
$$


B4. 4

where $\mathbf{s}=\sin \psi$ and $\psi$

is half the angle at

the apex of the normal

region. Assuming that

In the present model

the phase boundary near

the axis may be reason-

ably approximated by

a straight line, we

have used the Kuper

formula to find the

values of $\rho$ for dif-

fere:: diameters of

Indium wire assuming

$I=2.5 \times 10^{-3} \mathrm{~cm}$.

Our clirve is shown

in Fig. 4 along with

expertinental results for comparison.

It is obvious that, due to joule heating, a temperature

gradient will be set up along the radius of the wire, with the axial

region having the higher temperature and correspondingly lower $\mathrm{H}_{\mathrm{c}}$.

- We have found that even a small relaxation of the field condition

at the boundary near the centre of the wire has a marked effect on the shape of the boundary near the axis with a resultant increase in

p. This effect will clearly be greater both when $i_{c}$ is increased by lowerir.e the temperature and when the residual resistance is greater. References: Rếerence numbers are as given in (1): D. C. Baird and H. K. rukherjee. Phys. Letts. 25A (1967) 137.

In $\mathrm{F}_{i g}$. 4: R. Freud: $\mathrm{Cz}$. Sulkowski and B. Makiej, Phys. Letts. 27?A $(: 968) 187$. 


\title{
DESTRUCTION OF SUPERCONDUCTIVITY BY A CURRENT
}

\author{
D. C. Baird \\ Department of Physies, Royal Military College, Kingston, Ontario, Canada \\ and \\ B. X Mukherjee \\ Department of Physics, The University, St. Andrews, Fife, Scotland \\ (Received 19 August 1968)
}

\begin{abstract}
We present 2 new model of the intermediate state in currentmcarrying superconductors. The model predicts a resistance transition which is in reasonable agreement with experimental values. An introductory treatment of secondary effects is also given.
\end{abstract}

When superconductivity in a wire is destroyed by a current, resistance returns in a manner different from that predicted by London, ${ }^{1-4}$ and the consideration of secondary effects ${ }^{5,6}$ does not account satisfactorily for the discrepancy. We present a treatment which gives the structure of the intermediate state, predicts a resistance transition in reasonable agreement with experimental observations, and enables the evaluation of secondary effects.

When the current in a superconducting wire reaches the value $i_{C}$ at which the magnetic field at the surface has the critical value $H_{C}$, flux should start to penetrate the wire and create a series of normal regions along the wire. Throughout these regions the field must obviously be $H_{C}$ or as close to $H_{C}$ as possible. It follows that in the normal regions the variation of current density with radius $r$ should ideally have the form

$$
j(r)=\left(i_{c} / 2 \pi a\right)(l / r)
$$

where $a$ is the radius of the wire. However, it is not possible to find a finite structure which gives $H=H_{C}$ throughout the whole of the normal volumes, and the most important condition is obviously that $H=H_{C}$ at each normal-superconduct- ing interface.

For a given boundary the potential distribution in one of the normal regions can be obtained by a numerical solution of Laplace's equation subject to the following boundary conditions: (i) $V=$ constant on any $s-n$ boundary and (ii) $\partial V / \partial r=0$ at the surface of the wire. The current distribution, and hence the field distribution, can then be calsulated from the potential distribution. Using trial and error methods, boundaries satisfying the field criterion mentioned above can be found for any given ratio of the wire radius $a$ to the structure periodic length $d$, up to a limiting valne $(a / d)_{\max }=1.4$. The axial width of the normal regions at the center of the wire falls rapidly to zero as $a / d$ approaches 1.4 , thus making it impossible to find satisfactory boundaries for $a / d$ larger than 1.4 .

As has been pointed out by Shoenberg, ${ }^{7}$ two mutually opposing criteria will determine the optimum value of $a / d$ : (i) That throughout the normal region $H$ be as close as possible to $H_{C}$. Quantitatively, this amounts to minimizing

$$
\dot{e}=\frac{H^{2}}{8 \pi} V_{n}-\int_{V_{n}} \frac{H^{2}}{8 \pi} d v^{\prime}
$$




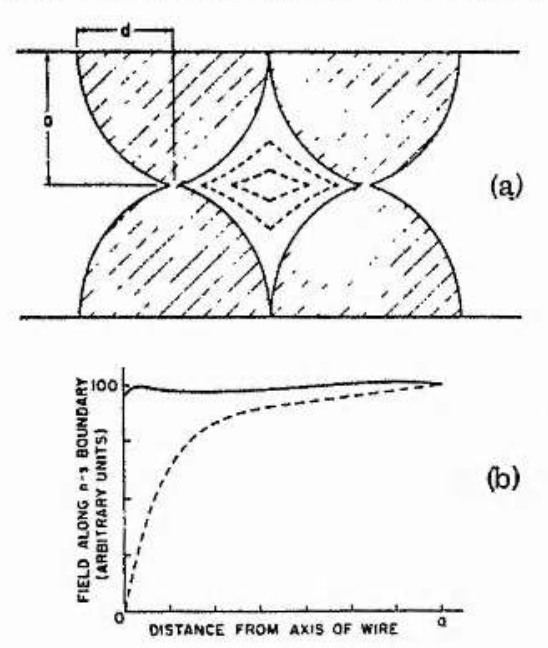

FIG. 1. (a) Intermediate-state structure for $a / d$ $=1.4$. (b) Variation of $H$ along the boundary compared with that of London's model.

per unit length of wire, where $V_{n}$ is the volume of the normal region. It is obvious that $e$ decreases as $a / d$ increases. (ii) The n-s surface energy $s$ per unit length of wire be a minimum. This makes smaller values of $a / d$ more favorable. Values of $e$ and $s$ have been calculated for indium and it has been found that for the values of $a / d$ under consideration, $s$ is only a few percent of $e$, and is therefore negligible. In type-I superconductors $s$ becomes important only when $a / d$ is much larger than 1.4. Hence, we have accepted $(a / d)_{\max }=1.4$ as the optimum value, and we suggest that at $i=i_{c}$ the structure of the intermediate state is as shown in Fig. 1(a). Such a structure has the normalized resistance

$$
\rho=\left(R / R_{n}\right)_{i=i_{c}}=0.69,
$$

and the variation of field along the n-s boundary is shown in Fig. 1(b).

When the current rises above $i_{c}$, the superconducting cores must shrink to an equilibrium shape for each value of current so as to maintain the condition $H=H_{C}$ along the boundaries. Boundaries to satisfy this criterion have been found for several values of $i>i_{c}$, and two of them are indicated by the broken lines in Fig. 1(a). The resistances of these structures have been evaluated, and the full return of resistance curve as predicted by our model is shown in Fig. 2.

Since the present model gives a definite size to the structure, it is amenable to the treatment of secondary effects such as mean free path and Joule heating.

In the above calculations we have assumed that

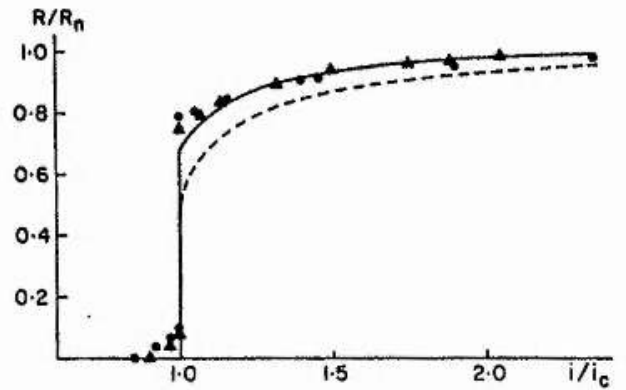

FIG. 2. Return of resistance curve: solid line, present model; dashed line, London (Ref. 1); closed circles, Scott (Ref. 2) (0.286-mm-diam in wire); closed triangles, D. C. Baird and B. K. Mukherjee (unpublished) $(0.5-\mathrm{mm}$-diam In wire).

the mean free path $l$ in the normal material is small compared with the dimensions of the normal regions. Kuper has suggested that when this is not the case, scattering of electrons at n-s interfaces will reduce the effective mean free path, thereby increasing the value of $\rho$. He has proposed $d^{6}$ that for the London model the average effective conductivity is given approximately by

$$
\sigma(r)=\sigma_{0}\{1-(1-p) \exp (-r p / l)\}
$$

with $p=\sin \psi$, where $\psi$ is half the angle at the apex of the normal region. Assuming that the lower part of the phase boundary in the present model may be reasonably approximated by a straight line, we have used the Kuper formula to find the values of $\rho$ for different diameters of pure indium wire assuming that $l=2.5 \times 10^{-3} \mathrm{~cm}$, and our results are shown in Fig. 3 along with experimental results for comparison.

It is obvious that due to Joule heating a temper-

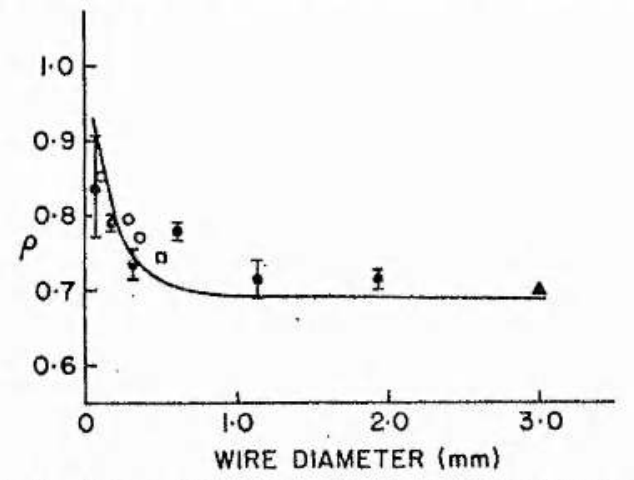

FIG. 3. Mean free path effect: The resistance discontinuity $\rho$ at $i_{C}$ plotted as a function of wire diameter. Solid line: prediction of the present model; open circles: Scott (Ref. 2), closed circles: Meissner and Zdanis (Ref. 4), open squares: D. C. Baird and B. K. Mukherjee (unpublished), and closed triangles:

R. Freud, Cz. Sulkowski, and B. Makie] [Phys, Letters 27A, $187(1968)]$. 
ature gradient will be set up along the radius of the wire with the axial region having the higher temperature and a correspondingly lower $H_{C}$. We have found that even a small relaxation of the field condition at the boundary near the center of the wire has a marked effect on the shape of the boundary near the axis, with a resultant increase in $\rho$. Thus, if $H_{C}$ near the center is about $25 \%$ less than at the surface of the wire, the resistance ratio increases by about $8 \%$. This effect will clearly be greater when (i) $i_{c}$ is increased by lowering the temperature, and (ii) the residual resistance is increased.

We wish to express our thanks to Professor J. F. Allen for many helpful discussions and suggestions, and to Dr.C. D. McKay of the Royal Military College Computing Center and Dr. A. J.
Cole of the St. Andrews University Computing Laboratory for their assistance with the numerical solutions.

\footnotetext{
${ }^{1}$ F. London, Superfluids (John Wiley \& Sons, Inc., New York, 1950), Vol. 1.

${ }^{2}$ R. B. Scott, J. Res. NatI. Bur. Std. (U.S.) 41,581 (1948).

${ }^{3}$ L. Rinderer, Helv. Phys. Acta 29, 339 (1956).

${ }^{4} \mathrm{H}$. Meissner and R. Zdanis, Phys. Rev. 109, 681 (1958).

${ }^{5}$ S. T. Berkovich and G. M. Lapir, Zh. Eksperim, i Teor. Fiz. 44, 1329 (1963) [translation: Soviet Phys. - JETP 17, 896 (1963)].

${ }^{6}$ C. G. Kuper, Phil. Mag. 43, 1264 (1952).

'D. Shoenberg, Superconductivity (Cambridge University Press, New York, 1952).
} 


\section{DESTRUCTION OF SUPERCONDUCTIVITY BY A CURRENT \\ B.K. Mukherjee*, H.D. Wiederick and D.C. Baird Department of Physics, Royal College, Kingston, Ontario, Canada}

\footnotetext{
*Part of this work was carried out at the School of Physical
} Sciences, University of St. Andrews, Scotland. 


\section{ABSTRACT}

We have revised our model of the intermediate state in current carrying Type-1 superconductors. Several other models have also been put forward. We compare these models on the basis of evidence from three different kinds of experiments. 
At LT 11 we presented a model ${ }^{1}$ for the current induced intermediate state in Type-1 superconducting wires. In developing that model we had used the criterion that, at equilibrium, the magnetic field along any normal-superconducting boundary should have the critical value $H_{C}$, and we had pointed out that for any finite structure the field within a normal region will be equal to or less than $\mathrm{H}_{\mathrm{C}}$ : thus the model called for a certain amount of supercooling. We have since improved the model in order to reduce the supercooling involved ${ }^{2}$. Fig. 1 shows the improved model: the intermediate state remains the same as that given in (1) for the case of critical current $\left(i=i_{c}\right)$; however, as the current increases the intermediate state core will shrink but it is not necessary for the axial periodicity of the structure to remain constant. The superconducting regions pack together in order to maintain the optimum $n-s$ boundary which allows $H$ to equal $H_{c}$ on the boundary. The supercooling called for by this model has been discussed in (2) and shown to be plausible under normal experimental conditions.

An alternative model for the current-induced intermediate state in Type 1 superconducting wires has been put forward recently by Andreev ${ }^{3}$. We discuss below three different kinds of experiments which enable one to discriminate between the validity of different models.

1. The Resistance Transition: In Fig: 2 we compare the resistance transition predicted by the present model with the 
London model ${ }^{4}$ and with experimental values obtained for "thick" wires (diameter $\gtrsim 1 \mathrm{~mm}$.$) . The secondary effects which occur$ for thinner wires have already been discussed in (1) and remain valid. The complete resistance transition given by Andreev's model depends on a parameter $\alpha$ which we cannot evaluate. However according to Andreev the value of the resistance jump $p$ which occurs at $i=i_{c}$ is given by

$$
\rho=0.5+0.64(\Delta / a)^{2 / 9}
$$

where $\Delta$ is the surface energy parameter and a is the radius of the wire; this gives $\rho=0.597$ and $\rho=0.585$ for pure Indium wire of $1.65 \mathrm{~mm}$ and $3 \mathrm{~mm}$ respectively as compared with the experimental value of 0.697 and 0.695 respectively ${ }^{5,6}$.

II. The Radius of the Intermediate State Core: This radius has been measured experimentally as a function of $\left(i / i_{c}\right)$ by Rinderer ${ }^{7}$. We have used numerical methods to evaluate the values of $\left(i / i_{c}\right)$ corresponding to any given core radius and, as shown in Fig. 3, the values obtained by Rinderer are in good agreement with the theoretical curve predicted by the present model. From the macroscopic description given by Andreev it would appear that the core radius in his model is the same as that given by London's model and, as may be seen in Fig. 3, this does not agree well with the experimental values. III. Concentration of llormal Phase: Following the method suggested by Wiederick ${ }^{3}$ we have carried out an experiment to find the concentration of normal phase as a function of $\left(i / i_{c}\right)$. In this the specimen wire is placed along the axis of a thin 
solenoid whose a.c. inductance is monitored as a slowly increasing d.c. current drives the snecimen through the intermediate state. In effect the small a.c. field (which distorts the d.c. field by about . $1 \%$ only) is used as a probe to "see" the normal regions in the wire and demagnetising effects can be taken account of by the use of numberical methods. Further details will be published elsewhere. Results, of a preliminary experiment are given in Fig. 4 where we show the change of inductance of the coil as the wire is driven through the intermediate state. The theoretical claculations taking account of the demagnetising effect can only be done if the physical structure of the $n$ and $s$ regions is known for any model. Since Andreev has not specified the structure and since we do not know the appropriate value of $\alpha$, we have restricted ourselves, in Fig. 4, to showing points which represent the actual normal phase concentrations given by the models. 


\section{References:}

1. B.K. Mukherjee, J.F. Allen and D.C. Baird, "Low Temperature Physics LT 11" (University of St. Andrews, Scotland, 1969) Vol.2,p. 327.

2. D.C. Baird and B.K. Mukherjee, To be published.

3. A.F. Andreev, Sov. Phys. J E T P 27 (1968), 809.

4. F. London, Superfluids (John Wiley \& Sons, Inc., New York, 1950) Vol. 1.

5. B.K. Mukherjee and D.C. Baird unpublished result.

6. R. Freud, Cz. Sulkoirski and B. Makiej, Phys. Letts. $27 \mathrm{~A}(7968), 187$.

7. 1. Rinderer, Helv. Phys. Acta 29(1956), 339.

8. H.D. Wiederïck, Submitted to J. Phys. D: App. Phys. 


\section{FIGURE CAPTIONS}

FIS. 1.

Structure of the intermediate state for three values of apnlied current: (a) $i=i_{e},(b) i=1.6 i_{e},(c) i=2.3 i_{e}$. superconducting regions are shaded.

FIG. 2 .

Return of resistance curve: Solid line, this model; dashed line, London (Ref. 4); closed circles, experimental results for $1.65 \mathrm{~mm}$. diameter In wires (Ref. 5); closed triangles, experimental results for $3 \mathrm{~mm}$. diameter $1 \mathrm{n}$. wires (Ref. 6).

FIG. 3.

Variation of the radius of the intermediate state core as a fraction of wire radius with applied current: solid line, this model; dashed line, London (Ref. 4); experimental values are those obtained by Rinderer (Ref. 7). FIG. 4.

Change of inductance of the solenoid as a slowly increasing current drives the wire from the fully superconducting to the fully normal state. Points represent normalized concentrations of normal phase as predicted by the various models. 


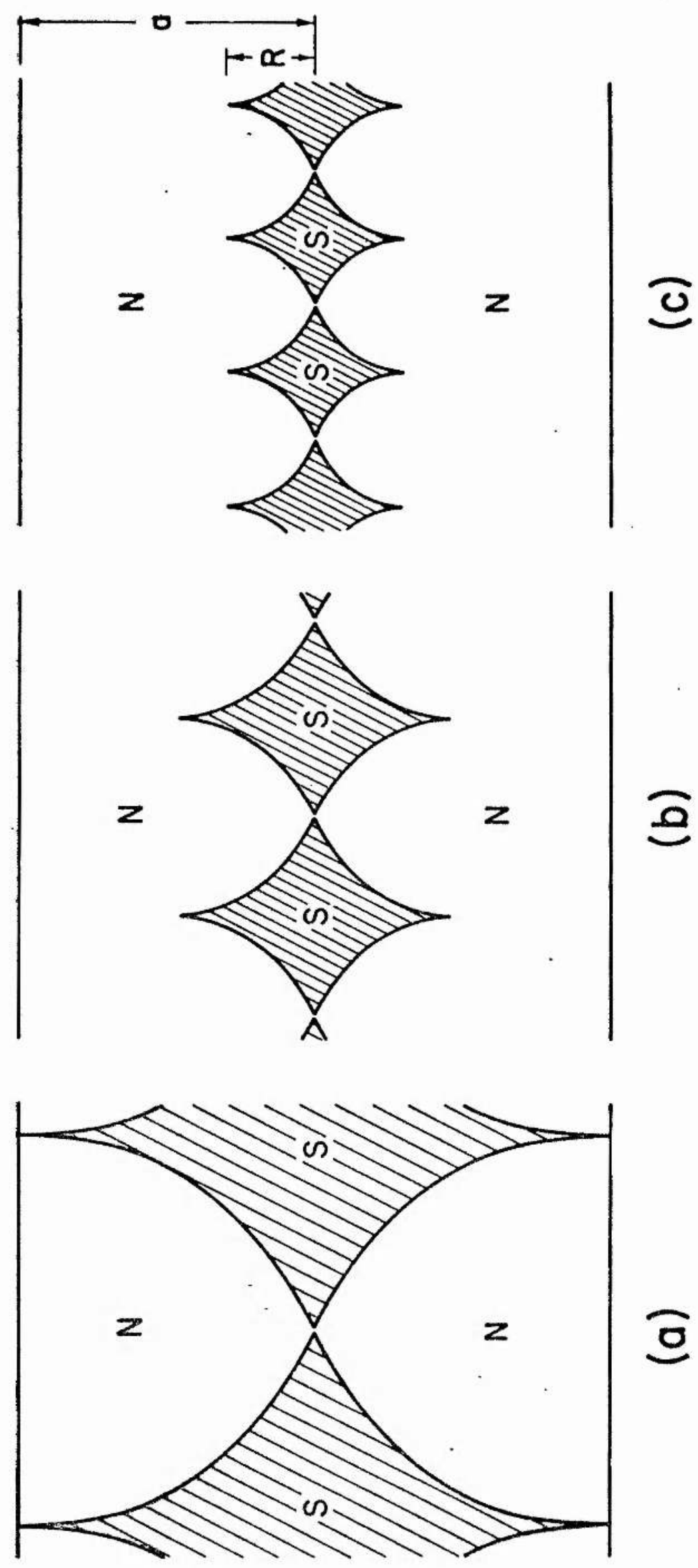




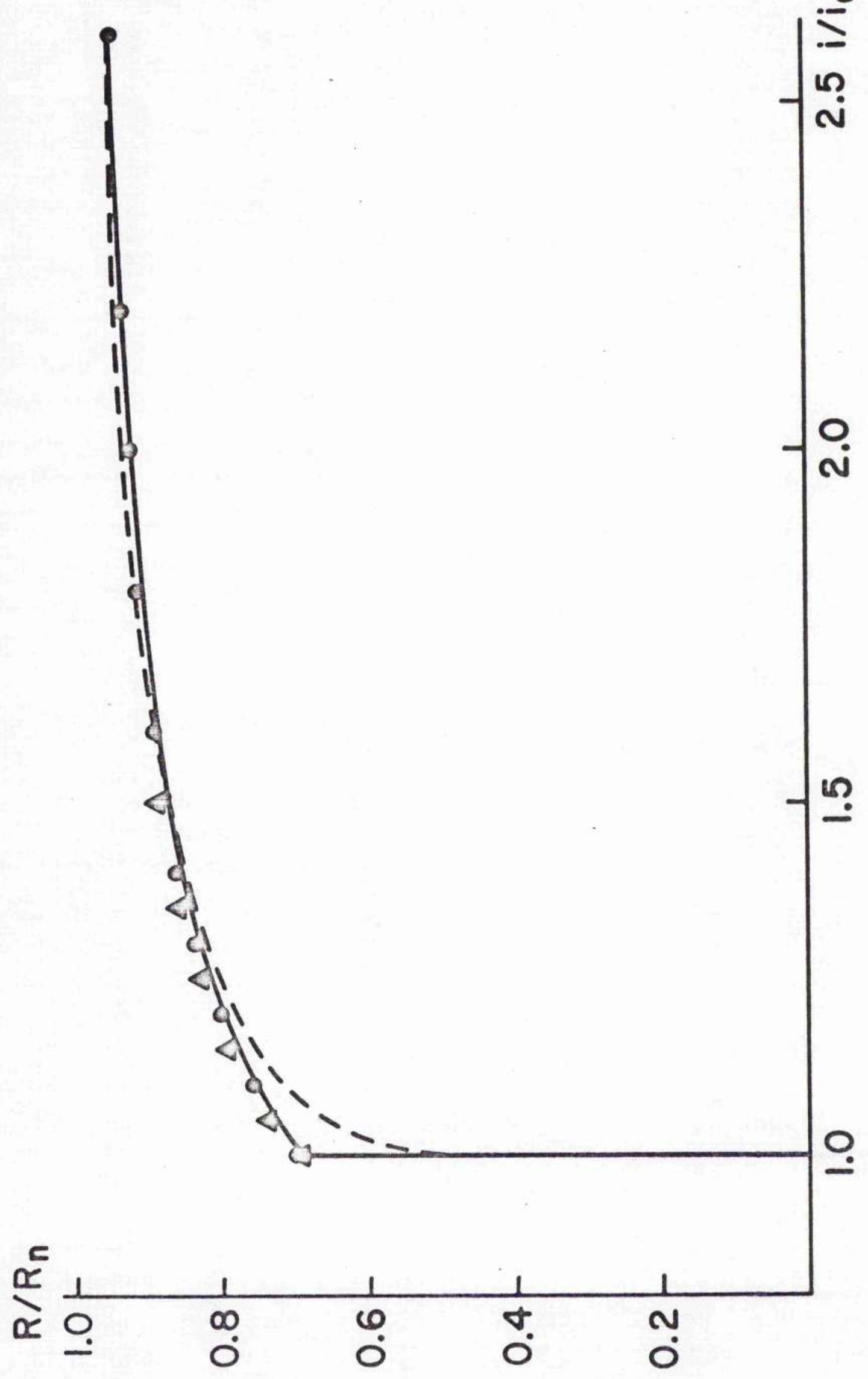




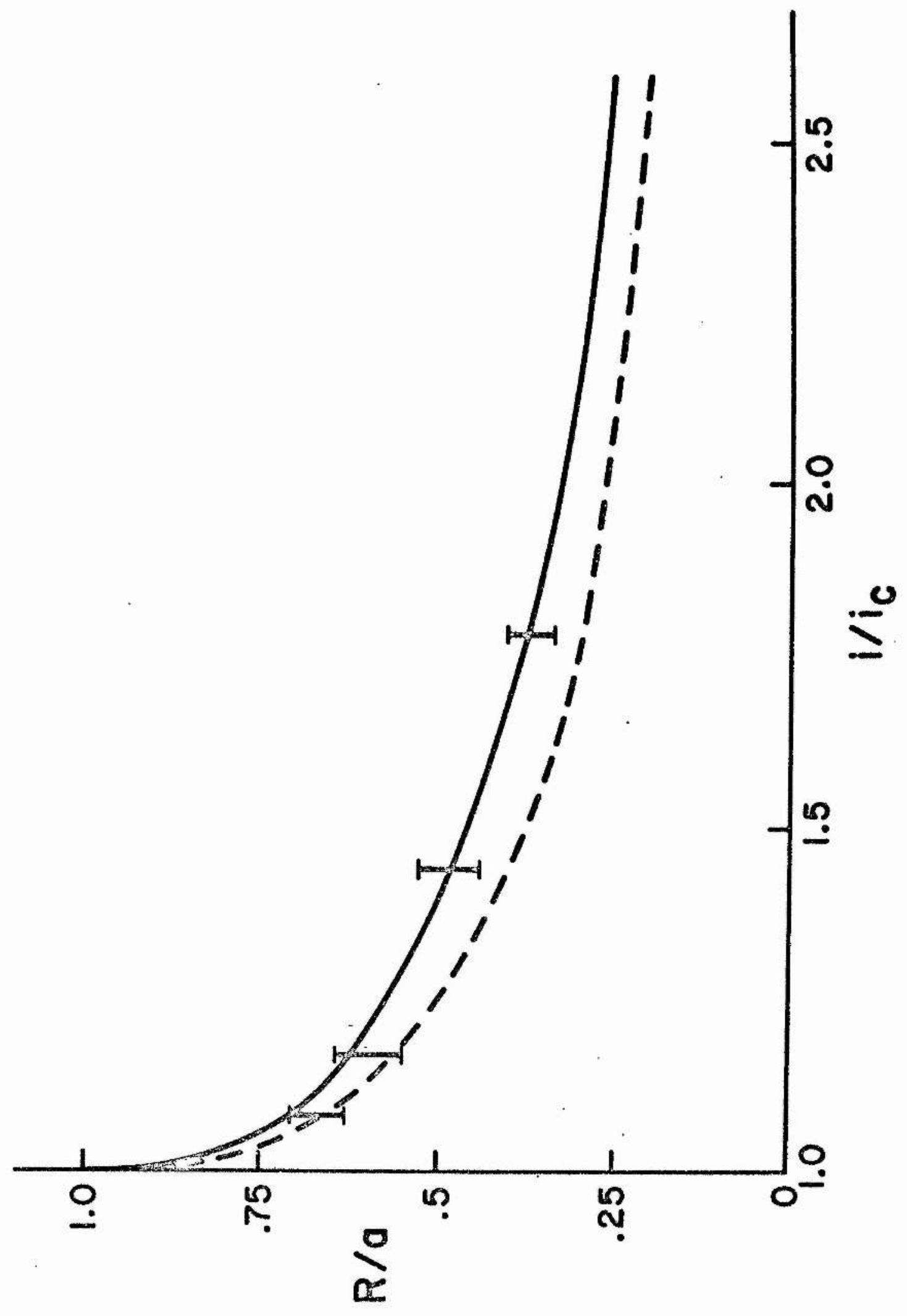




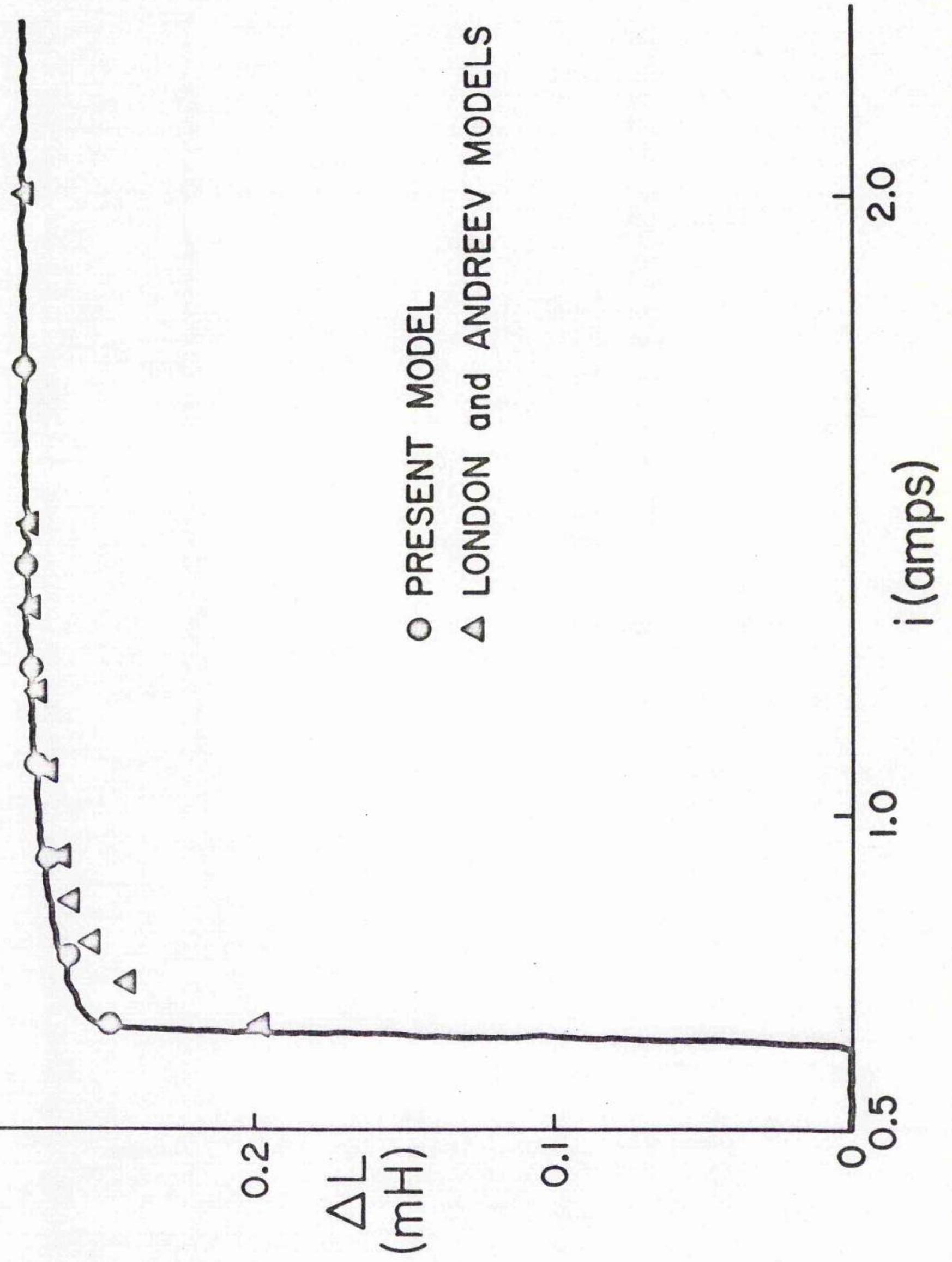




\title{
Current-Induced Intermediate State in Type-1 Superconductors
}

\author{
D. C. Baird \\ Department of Physics, Royal Military College, Kingston, Ontario, Canada \\ and \\ B. K. Mukherjee* \\ School of Physical Sciences, University of St. Andrews, St. Andrews, Fife, Scotland \\ (Received 23 July 1970)
}

We have improved our previous model of the intermediate state in type-1 current-carrying superconductors. In addition to predicting a resistance transition in reasonable agreement with experimental observations, the model gives good agreement with experimental values for the radius of the intermediate-state core as obtained by Rinderer.

In a previous paper ${ }^{1}$ (referred to hereafter as $\mathrm{BM}$ ) we presented a model of the intermediate state in current-carrying type-1 superconductors. The main theoretical criterion used was that, at equilibrium, the magnetic field $H$ at all points on a nor- mal-superconducting boundary should have the critical value $H_{c}$. Ideally the field should also be $H_{c}$ throughout the whole normal region, but it is not possible to find a finite structure to satisfy this ideal condition. Supercooling is therefore involved, since 
the structure gives values of $H$ in the normal regions less than $H_{c}$. We now present an improved model which produces less supercooling than the earlier model did and show that the supercooling required is acceptable.

The intermediate-state structure presented by $\mathrm{BM}$ for the case of critical current $\left(i=i_{c}\right)$ is shown in Fig. 1(a), and the magnetic field distribution in each normal region is indicated in Fig. 2. It can be seen that the supercooling required is maximum near the axis of the wire. We note, however, that on the axis itself an infinite current density would be required to produce the critical field $H_{c}$ and this is not only physically unrealistic but would also give rise to a temperature singularity. In practice it is reasonable to expect that the structure is such as to give a large, but finite, current de.ssity and a small finite increase in temperature near the axis. As a result, $H_{c}$ would have a smaller value near the axis, and hence the supercooling requirement there is diminished.

The question now is whether or not the supercooling indicated is plausible under normal experimental conditions. We note that most resistance transition experiments are carried out near $T_{c}$ and, under these conditions, Ginzburg ${ }^{2}$ has shown that the lower supercooling limit for an ideal type-1 superconductor is given by

$$
S_{i}=H_{\mathrm{sd}} / H_{Q}=\sqrt{2} \dot{\kappa},
$$

where $H_{\mathrm{Bc}}$ is the lowest field at which the normal phase can persist and $\kappa$ is the Ginzburg-Landau parameter. In agreement with this theory Faber ${ }^{3}$ experimentally found values of $S_{t} \sim 0.16$ for tin and $S_{l}$ $\sim 0.11$ for indium. Besides, recent experiments ${ }^{4}$ with extruded plycrystalline indium wires (similar to those used in resistance-transition experiments) give $S_{l} \sim 0.36$. Thus the supercooling required by the structure of Fig. 1(a) is well within acceptable limits.

BM suggest that when the current increases above $i_{e}$, the superconducting cores will shrink to an equilibrium shape for each value of current so as to

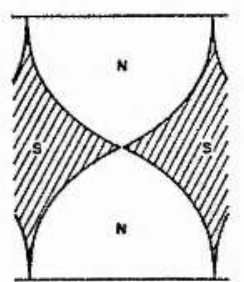

(a)

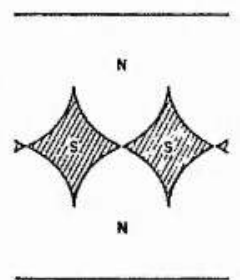

(b)

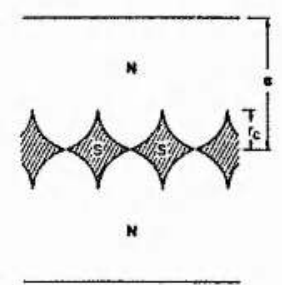

(c)
FIG. 1. Structure of the intermediate state for three values of applied current: (a) $i=i_{c}$, (b) $i=1.6 i_{c}$, (c) $i$ $=2.3 i_{c}$.
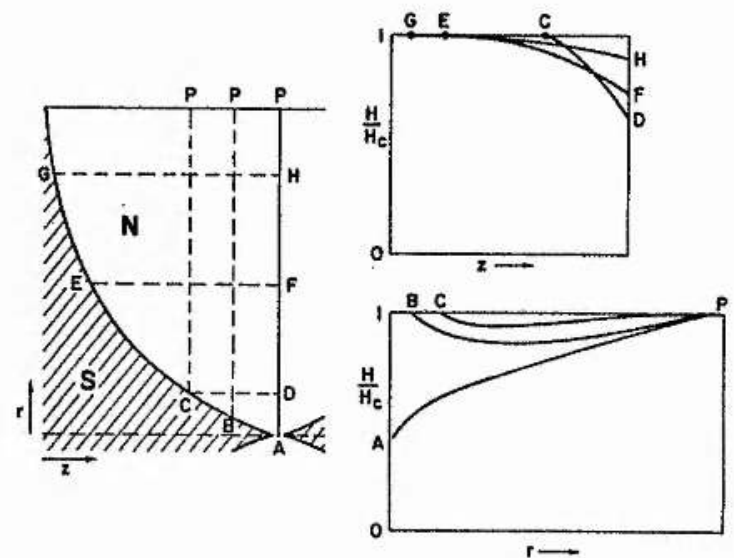

FIG. 2. Distribution of the magnetic field in a normal region. A quarter of the diametral section of a normal region is shown at left, and the field distribution along the lines AP, BP, $\mathrm{CP}$ and $\mathrm{CD}, \mathrm{EF}$, and $\mathrm{GH}$ is shown at right. $r$ represents a radial direction and $z$ the axial direction.
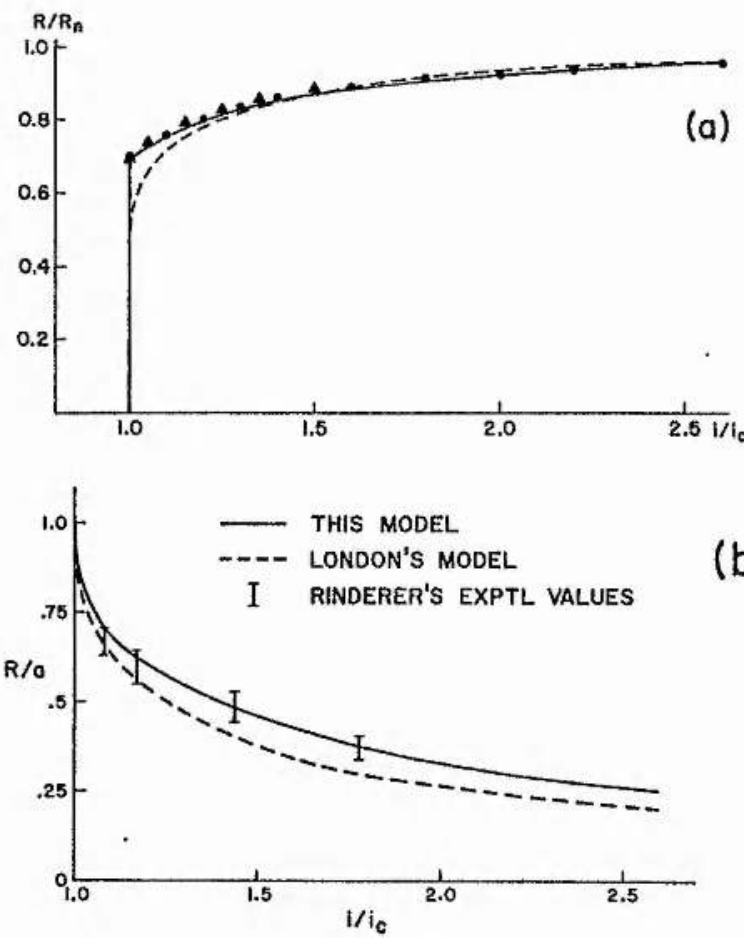

(b)

FIG. 3. (a) Return of resistance curve ( $R$ is actual resistance, $R_{n}$ is fully normal resistance): solid line, this model; dashed line, Ref. 5 ; closed circles, experimental values for $1.65-\mathrm{mm}$-diam In wire, D. C. Baird and B. K. Nukherjee (unpublished); closed triangles, experintental values for 3-mm-diam In wire, Ref. 6 . (b) Variation of the radius $r_{c}$ of the intermediate state core (see Fig. 1) as a fraction of wire radius $a$ with applied current: solid line, this model; dashed line, Ref. 5; experimental values, Ref. 7 . 
maintain the condition $H=H_{c}$ along the boundaries, and show two such structures. These structures indicate that, as the current increases, sodoes the axial width of the normal regions, and it follows that the supercooling required near the center of the normal regions would increase and become unacceptably large. An alternative possibility is shown in Fig. 1: As $i$ increases over $i_{c}$, a fully normal sheath is formed surrounding an intermediate state core: within the core, the structure will be determined by the same equilibrium conditions as are valid at $i=i_{c}$, and we may therefore assume that the axial periodicity of the structure decreases soas to maintain the optimum $n-s$ boundary shape which allows $H$ to equal $H_{c}$ on the boundary. It follows that the supercooling called for in the normal regions is not greater than it was at $i=i_{c}$.

In Fig. 3(a) the resistance transition predicted by the present model is compared with the London model and with experimental values obtained for "thick" wires (diameter $\gtrsim 1 \mathrm{~mm}$ ). For thinner wires secondary effects occur; these have been discussed by $\mathrm{BM}$ and the treatment given there remains valid.

In addition to the usual consideration of resistance transitions, an independent check on the validity of any model of the intermediate state in current-carrying type- 1 superconductors is provided by Rinderer's measurements of the radius of the intermediate-state core as a function of applied current. ${ }^{7}$ Figure $3(\mathrm{~b})$ shows that the values obtained by Rinderer are in good agreement with the present model, whereas they do not agree well with the values predicted by London.

We wish to express our thanks to Professor J. F. Allen for many helpful discussions and to Dr. A. J. Cole and the staff of the St. Andrews University computing laboratory for their assistance with the numerical solutions.

\footnotetext{
*Present address: Department of Physics, Royal Military College of Canada, Kingston, Ontario. Canada.

${ }^{1} D$. C. Baird and B. K. Mukherjee, Phys. Rev. Letters 21,996 (1968).

${ }^{2}$ V. L. Ginzburg, Zh. Elssperim. i Teor. Fiz. 34, 113 (1958). [Soviet Phys. JETP 7, 78 (1958)].

${ }^{3}$ T. E. Faber, Proc. Roy. Soc. (London) A241, 531 (1957).
}

${ }^{4}$ R. Deltour, H. N. De Lang, and P. Wyder, Phys. Letters 31A, 515 (1970).

${ }^{5}$ F. London, Superfluids (Wiley, New York, 1950), Vol. 1.

${ }^{6}$ R. Freud, C. Sulkowski, and B. Makiej, Phys. Letters 27A, 187 (1968).

${ }^{7}$ L. Rinderer, Helv. Phys. Acta $\underline{29}, 339$ (1956). 